\title{
Arabidopsis MLO2 is a negative regulator of sensitivity to extracellular reactive oxygen species
}

\section{Cui, Fuqiang}

2018-04

Cui , F , Wu , H , Safronov , O , Zhang , P , Kumar , R, Kollist , H , Salojärvi , J T , Panstruga , R \& Overmyer, K L 2018, ' Arabidopsis MLO2 is a negative regulator of sensitivity to extracellular reactive oxygen species ' , Plant, Cell and Environment, vol. 41 , no. 4 , pp. $782-796$. https://doi.org/10.1111/pce.13144

http://hdl.handle.net/10138/326147

https://doi.org/10.1111/pce.13144

unspecified

acceptedVersion

Downloaded from Helda, University of Helsinki institutional repository.

This is an electronic reprint of the original article.

This reprint may differ from the original in pagination and typographic detail.

Please cite the original version. 


\section{Arabidopsis MLO2 is a negative regulator of sensitivity to extracellular ROS}

2 Running title: Role of MLO2 in ozone- and abiotic stresses

3 Fuqiang Cui ${ }^{1}, 5$, Hongpo $\mathrm{Wu}^{2}$, Omid Safronov ${ }^{1}$, Panpan Zhang ${ }^{1}$, Rajeev Kumar ${ }^{3}$,

4 Hannes Kollist $^{4}$, Jarkko Salojärvi ${ }^{1}$, Ralph Panstruga ${ }^{2}$ and Kirk Overmyer ${ }^{1,{ }^{*}}$

$5{ }^{1}$ Division of Plant Biology, Department of Biosciences, Viikki Plant Science Centre,

6 University of Helsinki, 00014 Helsinki, Finland.

$7 \quad{ }^{2}$ Unit of Plant Molecular Cell Biology, Institute for Biology I, RWTH Aachen

8 University, 52056 Aachen, Germany.

$9 \quad{ }^{3}$ Department of Agricultural Biotechnology and Molecular Biology, Dr. Rajendra

10 Prasad Central Agricultural University, Pusa, Samastipur Bihar, India.

$11{ }^{4}$ Institute of Technology, University of Tartu, Nooruse 1, Tartu 50411, Estonia.

$12{ }^{5}$ Current address: State Key Laboratory of Subtropical Silviculture, Zhejiang 13 Agriculture \& Forestry University, Lin'an, Hangzhou 311300, China.

14

$15{ }^{*}$ Corresponding Author: Kirk Overmyer, Division of Plant Biology, Department of 16 Biosciences, Viikki Plant Science Centre, University of Helsinki, 00014 Helsinki, 17 Finland. E-mail:kirk.overmyer@helsinki.fi.

19 The authors have no conflict of interest to declare. 


\section{ABSTRACT}

The atmospheric pollutant ozone $\left(\mathrm{O}_{3}\right)$ is a strong oxidant that causes extracellular reactive oxygen species (ROS) formation, has significant ecological relevance, and is used here as a non-invasive ROS inducer to study plant signalling. Previous genetic screens identified several mutants exhibiting enhanced $\mathrm{O}_{3}$-sensitivity, but few with enhanced tolerance. We found that loss-of-function mutants in Arabidopsis MLO2, a gene implicated in susceptibility to powdery mildew disease, exhibit enhanced dosedependent tolerance to $\mathrm{O}_{3}$ and extracellular ROS, but a normal response to intracellular ROS. This phenotype is increased in a mlo2 mlo6 mlo12 triple mutant, reminiscent of the genetic redundancy of $M L O$ genes in powdery mildew resistance. Stomatal assays revealed that enhanced $\mathrm{O}_{3}$ tolerance in $\mathrm{mlo} 2$ mutants is not caused by altered stomatal conductance. We explored modulation of the mlo2-associated $\mathrm{O}_{3}$ tolerance, powdery mildew resistance, and early senescence phenotypes by genetic epistasis analysis, involving mutants with known effects on ROS sensitivity or antifungal defence. Mining of publicly accessible microarray data suggests that these MLO proteins regulate accumulation of abiotic stress response transcripts, while transcript accumulation of $\mathrm{MLO}_{2}$ itself is $\mathrm{O}_{3}$-responsive. In summary, our data reveal MLO2 as a novel negative regulator in plant ROS responses, which links biotic and abiotic stress response pathways.

\section{KEYWORD INDEX}

ROS signalling, MLO2, ozone, immunity, abiotic stress, unequal genetic redundancy 


\section{INTRODUCTION}

Mildew Locus $O(M L O)$ genes code for polytopic integral membrane proteins of unknown biological function (Acevedo-Garcia et al. 2014). Mlo was originally identified in barley (Hordeum vulgare), but is conserved in plants in small to medium-sized gene families (Büschges et al. 1997; Kusch et al. 2016). Loss-of-function alleles of specific $M L O s$ confer resistance against powdery mildew disease in various plant species, thus they are considered negative regulators of plant immunity or alternatively as "susceptibility" genes co-opted by powdery mildew pathogens to promote pathogenesis (van Schie, C. C. N. \& Takken 2014; Panstruga 2005). The Arabidopsis thaliana (Arabidopsis) genome contains a 15-member MLO family. MLO2 (At1g11310), MLO6 (At1g61560) and MLO12 (At2g39200) form a phylogenetic group (clade V MLOs) most similar to barley Mlo (Devoto et al. 2003). These genes cofunction with unequal redundancy in the regulation of immunity against Arabidopsis powdery mildew pathogens such as Golovinomyces orontii. Accordingly, mlo2 single mutants are partially and $\mathrm{mlo} 2 \mathrm{mlo6}$ mlo12 triple mutants fully resistant (Consonni et al. 2006; Acevedo-Garcia et al. 2017). Reminiscent of penetration resistance typical for non-host interactions (Humphry et al. 2006), immunity conferred by loss of MLO function is broad spectrum, durable, and halts fungal ingress prior to the sporeling breaching the host cell wall (Acevedo-Garcia et al. 2014). Penetration resistance is associated with deposition of cell wall appositions (papillae) at the attempted entry sites. Among other constituents, papillae contain callose, which locally reinforces the cell wall (Ellinger et al. 2013; Voigt 2014). Papilla formation often spatially and temporally coincides with an apoplastic burst of reactive oxygen species (ROS), which further strengthens cell walls by oxidative crosslinking (Hückelhoven et al. 2001; Mellersh et al. 2002). In addition to its immunity phenotypes, the Arabidopsis mlo2 
single and mlo2 mlo6 mlo12 triple mutants also exhibit premature leaf senescence with associated spontaneous, ectopic callose deposition and ROS accumulation (Consonni et al. 2006; Acevedo-Garcia et al. 2017; Consonni et al. 2010). Beyond MLO2, MLO6 and MLO12, few functions of other Arabidopsis MLO genes are known. MLO4 and MLO11 are involved in modulating root thigmomorphogenesis (Chen et al. 2009; Bidzinski et al. 2014) and MLO7 in the regulation of pollen tube growth (Kessler et al. 2010).

ROS act as versatile signalling intermediates in a variety of cellular pathways. Signalling specificity is determined largely by the site of ROS production and the chemical properties of each species, such as hydrogen peroxide $\left(\mathrm{H}_{2} \mathrm{O}_{2}\right)$, superoxide anion $\left(\mathrm{O}_{2}-\right)$, hydroxyl radical $(\mathrm{OH} \cdot)$, or singlet oxygen $\left({ }^{1} \mathrm{O}_{2}\right)$ (Gadjev et al. 2006). In plant development, ROS are involved in growth, specifically in cell wall loosening, cell expansion, and cell cycle progression (Gapper \& Dolan 2006; Swanson \& Gilroy 2010; Polyn et al. 2015). Other developmental events, such as fertilization, mechanosensing in thigmomorphogenesis, senescence, and developmentally programmed cell death are also regulated by ROS (Huysmans et al. 2017; Rogers \& Munné-Bosch 2016; Duan et al. 2014). Importantly, ROS signalling is a prominent feature in plant responses to both abiotic and biotic stresses. ROS act as signalling intermediates in the abscisic acid (ABA) pathway, a key regulator of stomatal closure and adaptation to heat, cold, desiccation, and osmotic stresses (Tuteja 2007). Additionally, ROS function in ABAindependent pathways in the response to these stresses. In plant immunity, defence responses are coordinated by a rapid and transient peak in ROS production termed the oxidative burst (Torres 2010). The oxidative burst is characteristic of multiple forms of disease resistance, playing an active role in basal defences, or so-called PAMPtriggered immunity (PTI), as well as coordinating the activation of defence signalling 
and cell death in gene-for-gene resistance, or effector-triggered immunity (ETI; Jones \& Dangl 2006). ROS production in plant immune responses occurs typically on the extracellular side, where plasma membrane NADPH oxidases, dubbed in plants as respiratory burst oxidase homologs $(\mathrm{RBOHs})$, or apoplastic peroxidases, produce superoxide into the apoplastic space (Qi et al. 2017).

Ozone $\left(\mathrm{O}_{3}\right)$ is an atmospheric pollutant and itself a form of ROS (Overmyer et al. 2009). Entering leaves through open stomata, $\mathrm{O}_{3}$ breaks down in the apoplast to form other ROS, which in turn trigger an active oxidative burst within the plant (Wohlgemuth et al. 2002). Thus, $\mathrm{O}_{3}$ exposure has been utilized as a tool to induce the oxidative burst, in the absence of other pathogen signals, for the study of downstream ROS signalling (Vainonen \& Kangasjärvi 2015). The level of sensitivity to $\mathrm{O}_{3}$ within Arabidopsis is subject to natural genetic variation (Brosché et al. 2010). In addition, a number of induced mutants with enhanced $\mathrm{O}_{3}$ sensitivity have been identified (Vainonen et al. 2015; Overmyer et al. 2008; Rao et al. 2002; Overmyer et al. 2000; Saji et al. 2017; Saji et al. 2008). These are defective in various physiological pathways that likely are interconnected functionally with each other (Overmyer et al. 2008). However, only few mutants with enhanced $\mathrm{O}_{3}$ tolerance are known (Booker et al. 2004; Overmyer et al. 2000; Joo et al. 2005).

Prompted by apparent links of MLO proteins to physiological processes involving ROS, we took a genetic approach using $\mathrm{O}_{3}$ exposure to address the role of MLOs in ROS signalling. 


\section{MATERIALS AND METHODS}

118

119

120

121

122

123

124

125

126

127

128

129

130

131

132

133

134

135

136

137

138

139

140

\section{Plant growth}

Arabidopsis thaliana (Arabidopsis) was grown on 1:1 mixture of peat (B2, Kekkilä, Finland) and vermiculite with 5 plants per $8 \times 8 \mathrm{~cm}$ pot. Plants were cultivated in controlled environment chambers (Weiss 1300; Weiss Technik UK, Loughborough, U.K.) under short day conditions (12 h light/12 h dark) with $250 \mu \mathrm{mol} \mathrm{m}^{-2} \mathrm{~s}^{-1}$ irradiance at $22 / 18^{\circ} \mathrm{C}$ (day/night) and $70 / 90 \%$ relative humidity. Mutant seeds were obtained from the Nottingham Arabidopsis Stock Centre (NASC; www.arabidopsis.info) unless otherwise indicated. Double mutant genotypes were confirmed by PCR in the $F_{1}, F_{2}$, and $F_{3}$ generations prior to use. Mutants and primer sequences for genotyping are listed in Table S1A. Barley (ㅂordeum vulgare) was cultivated in a controlled growth room under $18 / 14^{\circ} \mathrm{C}$ day/night temperatures, with $60 \%$ constant humidity, $18 \mathrm{~h}$ days, and $450 \mu$ mole $\mathrm{m}^{-2} \mathrm{~s}^{-1}$ irradiance. The near-isogenic genotypes included in this study comprised wild type (Mlo) and mlo-1 mutant plants in the genetic background of cultivar Ingrid. The X-ray-induced mlo-1 loss-of-function mutation causes an amino acid exchange in Mlo (tryptophan 162 to arginine) that leads to an unstable protein variant (lack of detectable protein accumulation; Freisleben 1942; Büschges et al. 1997; Müller et al. 2005).

\section{ROS treatments}

For all ROS treatments, 15- to 25-day-old plants were used. ROS-treated and control plants were grown under identical conditions and randomly selected into experimental groups. Standard $\mathrm{O}_{3}$ exposures comprised $250 \mathrm{nl} \mathrm{I}^{-1}$ to $400 \mathrm{nl} \mathrm{I}^{-1} \mathrm{O}_{3}$ for $6 \mathrm{~h}$ unless otherwise noted, with parallel "clean air" controls as described (Overmyer et al. 2000). "Clean air" controls were exposures to unfiltered ambient air with normal background 
$141 \mathrm{O}_{3}$ concentrations (typically $10-20 \mathrm{nl} \mathrm{I}^{-1}$ ), which are known not to have any effect on 142 plants. In vitro superoxide exposures were generated enzymatically with xanthine $143(0.5 \mathrm{mM})$ and xanthine oxidase 0.1 unit $\mathrm{ml}^{-1}$; Sigma-Aldrich, Munich, Germany) in 144 sodium phosphate buffer $(10 \mathrm{mM}, \mathrm{pH} 7.0)$ delivered by vacuum infiltration into 145 detached, fully expanded middle-aged Arabidopsis leaves, or $3 \mathrm{~cm}$ segments of ten146 day-old barley primary leaves, and incubated $8-20 \mathrm{~h}$ at $22^{\circ} \mathrm{C}$ in closed $50 \mathrm{ml}$ tubes, as 147 described (Overmyer et al. 2000; Jabs et al. 1996).

148 ROS-induced cell death was measured by electrolyte leakage of 2-5 detached leaves 149 into $5 \mathrm{ml}$ or whole rosettes into $12 \mathrm{ml}$ milli-Q water for $1 \mathrm{~h}$ followed by quantification 150 with a conductivity meter (Model FE30; Mettler Toledo, Gießen, Germany). Electrolyte leakage data is expressed as \% total ions (determined after disrupting leaves by boiling) and are the means of 5-10 replicates. Methyl viologen response was measured 153 from eight- to ten-day-old in vitro seedlings aseptically grown on 0.5x MS media agar $154(0.8 \%$ agar, $0.05 \%$ MES, at $\mathrm{pH} 5.7$, and $1 \%$ sucrose $)$ in the presence or absence of 155 methyl viologen (0.01 $\mu \mathrm{M}$; Sigma; www.sigmaaldrich.com). Seedlings were grown in the vertical position and photographed with a size reference, root length was then quantified from photos using ImageJ software (https://imagej.nih.gov/ij/)

\section{Stomatal measurements}

Steady state stomatal conductance and photosynthesis rate was measured under controlled conditions using a GFS-3000 gas exchange system (Walz, Effeltrich,

161 Germany) using a whole Arabidopsis rosette cuvette. Stomatal conductance was also 162 measured using a Delta-T Device porometer with a clip on cuvette (Model AP4; www.delta-t.co.uk). For $\mathrm{O}_{3}$-induced stomatal closure and the diurnal stomatal aperture experiments, gas-exchange was monitored with a custom-built gas-exchange device, 
165

166

167

168

169

170

171

172

173

174

175

176

177

178

179

180

181

182

183

184

185

186

187

188

as previously described (Kollist et al. 2007). Briefly, plants were inserted to experimental chambers, when stomatal conductance had stabilized at $\sim 65-75 \%$ relative humidity and $100 \mu \mathrm{mol} \mathrm{m} \mathrm{m}^{-2} \mathrm{~s}^{-1}$ light, $\mathrm{O}_{3}\left(400 \mathrm{nl} \mathrm{l}^{-1}\right.$ for $\left.3 \mathrm{~min}\right)$ was applied. Water loss was measured by monitoring the weight of detached leaves.

\section{qRT-PCR analysis}

Control ("clean air") and $\mathrm{O}_{3}$-treated plants (10-15 plants each) were harvested at $1 \mathrm{~h}$ of $\mathrm{O}_{3}$ exposure $\left(350 \mathrm{nll}^{-1}\right)$. RNA was extracted according to the manufacturer's instructions (GeneJET kit; Thermo Fisher Scientific, Bartlesville, OK, USA). DNaseltreated RNA (3 $\mu \mathrm{g}$ ) was used for reverse transcription with RevertAid Premium Reverse Transcriptase (Thermo Fisher Scientific). A $25 \mu$ cDNA synthesis reaction was diluted to $100 \mu \mathrm{l}$, and $1 \mu \mathrm{l}$ thereof used as a template in the HOT FIREPol EvaGreen qPCR Mix (Solis BioDyne, Tartu, Estonia) with three technical replicates. Primer sequences are given in Table S1B. The raw cycle threshold values were analysed in qBase (Hellemans et al. 2007) using three reference genes (Table S1B).

\section{GO enrichment and cluster analysis}

Lists of genes misregulated in the mlo2 mutant previously identified by Consonni et al. (Consonni et al. 2010) were analysed using Gene Ontology Consortium Online enrichment analysis tool (www.geneontology.org), using Bonferroni correction for multiple testing. (Yi et al. 2013). Bootstrapped Bayesian Hierarchical Clustering was conducted as described (Wrzaczek et al. 2010). MLO2-regulated genes (Table S2A; sourced from Consonni et al. 2010), were subjected to clustering analysis with publicly available transcriptomics datasets as described previously (Blomster et al. 2011; Brosché et al. 2014). Affymetrix gene expression data accession numbers and preprocessing was described previously (Georgii et al. 2012). Briefly, data from 
189

190

191

192

193

194

195

196

197

198

199

200

201

202

203

204

205

206

207

208

209

210

211

ArrayExpress (www.ebi.ac.uk/microarrayas/ae/), Gene Expression Omnibus (www.ncbi.nlm.nih.gov/geo/), The Integrated Microarray Database System (http://ausubellab.mgh.harvard.edu/imds/) and NASC Arrays (http://arabidopsis.info/affy) were used. Differential expression profiles were computed from $\log _{2}$ ratios of mean treatment and control expression across biological replicates, following Robust Multi-array Average (RMA) normalization. Profiles were combined with custom oligo microarray data (Array Express accession E-MTAB-662), using preprocessing as described previously (Blomster et al. 2011; Brosché et al. 2014).

\section{Powdery mildew infection assays}

Powdery mildew infection assays were performed as described (Acevedo-Garcia et al. 2017). Briefly, ca. six-week-old plants grown in short day conditions (8 h light, $22^{\circ} \mathrm{C}$; $16 \mathrm{~h}$ dark, $20^{\circ} \mathrm{C} ; 80 \mu \mathrm{mol} \mathrm{m}{ }^{-2} \mathrm{~s}^{-1}$ ) were heavily inoculated with $\underline{G}$. orontii by leaf-to-leaf contact. At $48 \mathrm{~h}$ post inoculation, host cell entry rates per germinated conidiospore were microscopically assessed by the presence of secondary hyphae.

\section{Callose staining}

Plants were grown in short day conditions ( $8 \mathrm{~h}$ light, $22^{\circ} \mathrm{C} ; 16 \mathrm{~h}$ dark, $20^{\circ} \mathrm{C} ; 80 \mu \mathrm{mol}$ $\mathrm{m}^{-2} \mathrm{~s}^{-1}$ ) in a controlled environment until the age of five, six, seven or eight weeks. Rosette leaves were harvested and callose staining performed as previously described (Adam \& Somerville 1996). Epifluorescence microscopy was performed with a Keyence BZ-9000 digital microscope (Keyence, Düsseldorf, Germany) and a UV filter set. Each leaf sample was semi-quantitatively assigned to one of the following four categories: 0 , no signs of callose deposits; 1 , sporadic occurrence of callose deposits; 2, prevalent occurrence of callose deposits; 3 , massive occurrence of callose deposits. 
212 The experiment was performed four times, with three-four plants per genotype and 213 age.

\section{Statistical analysis}

215 Single representative experiments were tested by two-way ANOVA with post-hoc analysis using Tukey's honest significant difference test, or a T-test for pairwise comparisons. Pooled data from several independent biological repeats were fitted in a

218 linear model by genotype, treatment and their interaction terms. Model contrasts and 219 their significances were estimated with multcomp package in $\mathrm{R}$ (Version 3.03; Bretz et 220 al. 2011). Gene expression and percentage data were log-transformed prior to 221 statistical analysis.

\section{RESULTS}

\section{The mlo2 mutant exhibits enhanced tolerance to extracellular ROS}

224

To explore a potential role of MLO2 in the ROS signalling, we exposed Col-0 wild-type and mlo2-5 mutant plants (in the genetic background of Col-0) to $\mathrm{O}_{3}$, which induces extracellular ROS production. Plants were subjected $350 \mathrm{nl} \mathrm{I}^{-1} \mathrm{O}_{3}$, which caused visible lesions in Col-0 as well as in mlo6-2 and mlo12-1, but not mlo2-5 mutants (Figure 1a). To quantify this response, we measured electrolyte leakage as a proxy for cell death in $\mathrm{O}_{3}$-exposed plants, including mlo6-2 and mlo12-1 mutants, as additional candidates, and the highly sensitive radical induced cell death1-4 (rcd1-4) mutant (Overmyer et al. 2000), as a control (Figure 1b). In this assay, the mlo6-2 mutant had a sensitivity level similar to Col-0 wild-type ( 10\% ion leakage), while mlo2-5 was significantly more tolerant, with lower levels of $\mathrm{O}_{3}$-induced electrolyte leakage ( $6.5 \%$ ion leakage). As expected, the rcd1-4 mutant showed strongly enhanced $\mathrm{O}_{3}$ sensitivity $(\sim 26 \%$ ion leakage). The mlo12-1 mutant was moderately $\mathrm{O}_{3}$-sensitive in this experiment, 
exhibiting electrolyte leakage levels higher than Col-0 but lower than rcd1-4 ( 18\% ion leakage; Figure 1b).

\section{The enhanced ROS tolerance phenotype is specific for $\mathbf{m l o 2}$}

T-DNA/transposon knockout mutants are available for all fifteen Arabidopsis $M L O$ paralogs, except MLO8 (Consonni et al. 2006). To address whether the observed enhanced $\mathrm{O}_{3}$ tolerance is specific for mlo2-5, their ROS sensitivity was tested and quantified by electrolyte leakage in $\mathrm{O}_{3}$ exposures at $350 \mathrm{nl} \mathrm{I}^{-1}$, revealing that only mlo25 conferred significantly enhanced ROS tolerance ( $5 \%$ ion leakage as compared to $\sim 13 \%$ ion leakage for Col-0; Figure S1a). Additionally, some mlo mutants (mlo4-4, mlo7-1, mlo9-2 and mlo12-1) showed a tendency towards slightly increased $\mathrm{O}_{3}-$ sensitivity in this experiment ( $>16 \%$ ion leakage). The enhanced tolerance phenotype of mlo2 was further confirmed by testing the ROS sensitivity of eight independent mlo2 alleles, including three different T-DNA insertions and five ethyl methane sulfonate (EMS)-derived lines leading to various missense mutations in MLO2. All examined mlo2 mutants showed a similar level of ion leakage ( 4-8\%), which was clearly lower than that of the Col-0 wild-type control ( 17\%; Figure S1a).

\section{The $\mathrm{O}_{3}$ tolerance phenotype is dose-dependent and further enhanced in the $\mathrm{mlo} 2$}

\section{mlo6 mlo12 triple mutant}

To characterize further the role of MLO2 in the $\mathrm{O}_{3}$ response, electrolyte leakage was monitored in a dose-response experiment $\left(0,250,300,350\right.$ and $\left.400 \mathrm{nl} \mathrm{l}^{-1} \mathrm{O}_{3}\right)$, confirming the high sensitivity of the control rcd1-4, moderate sensitivity of mlo12-1, and enhanced tolerance of mlo2-5 (Figure 1c). In this experiment, the mlo6-2 mutant exhibited $\mathrm{O}_{3}$ tolerance similar to mlo2-5 at lower concentrations and tolerance intermediate to mlo2-5 and Col-0 at $400 \mathrm{nl} \mathrm{I}^{-1}$ (Figure 1c). The observed trend towards 
260

261

262

263

264

265

266

267

268

269

270

271

272

273

274

275

276

277

enhanced $\mathrm{O}_{3}$-tolerance in mlo6-2 suggests possible genetic redundancy between $M L O$ paralogs in the regulation of extracellular ROS responses. MLO2, MLO6 and MLO12 form a phylogenetic clade of Arabidopsis co-orthologs with the highest sequence similarity to barley Mlo and were previously shown to exhibit unequal genetic redundancy in the regulation of immunity against powdery mildew disease (Consonni et al. 2006). To test for potential functional overlap in the ROS response, mlo2-5, mlo62, mlo12-1 and the respective triple mutant ( $m / 02-5$ mlo6-2 mlo12-1) were exposed to very high $\mathrm{O}_{3}$ levels $\left(400\right.$ and $\left.500 \mathrm{nl} \mathrm{l}^{-1}\right)$. At $400 \mathrm{nl} \mathrm{I}^{-1}$, mlo2-5 and the triple mutant were more tolerant, exhibiting lower electrolyte leakage levels ( $7-8 \%$ as compared to $\sim 19 \%$ for Col-0 wild-type; Figure 1d). The $500 \mathrm{nl} \mathrm{I}^{-1}$ exposure exceeded the damage threshold for mlo2-5, which resulted in high electrolyte leakage ( 25\%). However, the mlo2-5 mlo6-2 mlo12-1 triple mutant remained undamaged at this $\mathrm{O}_{3}$ concentration, as seen by its low level ( $8 \%$ ) of electrolyte leakage, indicating it has a higher ROS tolerance than the mlo2-5 single mutant (Figure 1d).

\section{Enhanced tolerance to extracellular ROS is not restricted to $\mathrm{O}_{3}$ and also occurs} in a barley mlo mutant

To test whether the observed phenotype is specific for exposure to $\mathrm{O}_{3}$ or more general for elevated ROS levels, we monitored cell death as electrolyte leakage in leaves treated in vitro with an enzymatic superoxide generation system comprised of xanthine and xanthine oxidase (XXO; Overmyer et al. 2000). In this assay, Col-0 exhibited $\sim 60 \%$ electrolyte leakage, while the mlo2-5 single mutant and the mlo2-5 mlo6-2 mlo12-1 triple mutant showed a reduction in ion leakage $(\sim 50 \%$ and $\sim 45 \%$, respectively; Figure 2a). To address the question whether the elevated ROS tolerance is a specific feature of Arabidopsis mlo2 plants or a more general phenomenon of mlo mutants, we included a barley ( $\underline{\text { Hordeum vulgare }})$ mlo mutant in our experiments. $\mathrm{O}_{3}$ exposure and 
XXO treatment of wild-type (Mlo) cultivar Ingrid and a near-isogenic mlo null mutant (mlo-1) revealed that similar to the results in Arabidopsis, the mlo mutant genotype exhibited lower levels of ozone-induced visible damage and XXO-induced cell death, $\sim 23 \%$ ion leakage for $m l o-1$ versus $\sim 36 \%$ for wild-type Mlo (Figure S2).

\section{mlo2 mutants show an unaltered response to intracellular ROS}

To analyse the response of the Arabidopsis mlo mutants to intracellular ROS production, ten-day-old seedlings were grown in the presence and absence of the herbicide methyl viologen (MV), which induces light-dependent ROS production in the chloroplast. As a positive control, we included the rcd1-4 mutant, which shows enhanced tolerance to intracellular ROS (Ahlfors et al. 2004a). Results of these root growth assays revealed no difference between Col-0, the mlo2-5 single mutant and the mlo2-5 mlo6-2 mlo12-1 triple mutant (root length each $~ 10 \%$ of the controls as compared to $\sim 48 \%$ for rcd1-4; Figure $2 \mathrm{~b}$ ), suggesting that these MLOs specifically modulate responses to extracellular ROS, but not intracellular (chloroplast-derived) ROS.

\section{mlo2 mutants show unaltered stomatal responses upon $\mathrm{O}_{3}$ exposure}

$\mathrm{O}_{3}$ enters leaves through open stomata; thus stomatal mutant phenotypes may result in an altered $\mathrm{O}_{3}$ response (Brosché et al. 2010). To assess the potential contribution of stomatal aperture to the $\mathrm{O}_{3}$ tolerance phenotype of the mlo2 mutant, we measured a number of stomata-related phenotypes. The stomatal conductance of Col-0, the mlo2-5 single mutant and the mlo2-5 mlo6-2 mlo12-1 triple mutant was first tested under control growth conditions using a clip-on cuvette. In this experiment, we deployed the ost1-3 mutant (Vahisalu et al. 2010), which has constitutively open stomata, as a positive control. This line showed the expected elevated stomatal 
conductance, and a respective mlo2-6 ost1-3 double mutant was not significantly different from the ost1-3 single mutant $\left(\sim 600 \mu \mathrm{mol} \mathrm{H}_{2} \mathrm{O} \mathrm{m} \mathrm{m}^{-2} \mathrm{~s}^{-1}\right.$ each), while the mlo2-

3116 single mutant and the mlo2-5 mlo6-2 mlo12-1 triple mutant revealed a wild-type-like 312 stomatal conductance of $\sim 200 \mu \mathrm{mol} \mathrm{H}_{2} \mathrm{O} \mathrm{m}^{-2} \mathrm{~s}^{-1}$ (Figure 3a). We extended this analysis 313 using cuvettes able to fit an entire Arabidopsis rosette. The photosynthesis rate and 314 stomatal conductance were tested for Col-0, the mlo2-6 single mutant and the mlo2-5 mlo6-2 mlo12-1 triple mutant, which revealed no differences between genotypes (Figure S4a and b, respectively). Additionally, we assessed the diurnal cycle of stomatal conductance over a $24 \mathrm{~h}$ period with Col-0 and three mlo2 null mutant alleles (mlo2-5, mlo2-6 and mlo2-7), yielding comparable curves (Figure S4c). To test $\mathrm{O}_{3}$ induced stomatal closure, we monitored stomatal conductance in intact whole plants 320 following a single 3 min pulse of $\mathrm{O}_{3}\left(400 \mathrm{nl} \mathrm{I}^{-1}\right.$; Kollist et al. 2007), which resulted in similar stomatal closure kinetics in mlo2-5 and Col-0 wild-type plants (Figure 3b). Water loss from detached leaves, which is another common assay for induced stomatal closure, also revealed no differences between the wild-type, the mlo2-5 single mutant and the mlo2-5 mlo6-2 mlo12-1 triple mutant (water loss $~ 25 \%$, as compared to $\sim 70 \%$ in ost1-3; Figure $3 \mathrm{c}$ ). Taken together, these data demonstrate that mlo2 mutants have normal stomatal responses, excluding the possibility that the enhanced tolerance of mlo2 mutants to extracellular ROS is conditioned at the level of stomatal aperture.

Modulation of $\mathrm{O}_{3}$ phenotypes by $\mathrm{MLO2}$ in selected double mutants

The enhanced $\mathrm{O}_{3}$ tolerance phenotypes of mlo2 mutants is only seen at very high $\mathrm{O}_{3}$ concentrations ( $\geq 300 \mathrm{nl} \mathrm{I}^{-1}$; Figure $1 \mathrm{c}$ and d) that are arguably not physiologically 332 relevant. To evaluate the effect of $\mathrm{MLO} 2$ at lower $\mathrm{O}_{3}$ concentrations, double mutants 333 of mlo2-6 were created with the ROS $\left(\mathrm{O}_{3}\right)$-hypersensitive mutants, $r c d 1-4$ (Jaspers et 
al. 2009), reticulata-8 (re-8; Overmyer et al. 2008), open stomata1-3 (ost1-3, also known as snrk2.6 and snrk2e; Vahisalu et al. 2010) and vitiminc1-1 (vtc1-1; Conklin et al. 1997). Following exposure to $250 \mathrm{nl} \mathrm{l}^{-1} \mathrm{O}_{3}$, which induced cell death in re-8, ost1-3 and vtc1-1, there was no statistically significant difference between Col-0 wild-type plants and the mlo2-6 mutant. However, presence of the mlo2-6 mutation conferred a reduction in ROS sensitivity in the respective double mutants with re-8, ost1-3 and vtc1-1 (Figures 4a and S5). Unexpectedly, the mlo2-6 mutation enhanced the ROS sensitivity of the rcd1-4 mutant under these conditions, with the mlo2-6 rcd1-4 double mutant showing significantly more $\mathrm{O}_{3}$-induced electrolyte leakage ( 25\%) than $r c d 1-4$ ( 18\%; Figure 4a and S5). Jasmonic acid (JA)-insensitive mutants exhibit strongly enhanced sensitivity to $\mathrm{O}_{3}$ exposure (Overmyer et al. 2000). Thus, double mutants of mlo2-11 with mutants jasmonate resistant1 (jar1-1) and coronatine insensitive1-16 (coi1-16), which are defective in JA signalling (Ellis \& Turner 2002; Staswick et al. 1992), were also tested. The mlo2-11 mutation conferred enhanced ROS tolerance in the context of these JA signalling mutants, indicated by a dramatic reduction in electrolyte leakage induced at $250 \mathrm{nl} \mathrm{I}^{-1} \mathrm{O}_{3}$, in both the mlo2-11 jar1-1 and mlo2-11 coi1-16 double mutants (Figure 4b and S5). No role for ethylene signalling was found using the ethylene insensitive2 (ein2-1; Alonso et al. 1999) double mutant; mlo2-11 ein2-1 retained enhanced ROS tolerance (Figure 4b and S5). Taken together, these results indicate that (1) the threshold for MLO2-dependent effects can be lowered when the mlo2 mutation is in $\mathrm{O}_{3}$-sensitive mutant backgrounds; (2) that mlo2 is an enhancer of rcd1-4-associated ROS sensitivity; and (3) that JA signalling is not required for enhanced ROS tolerance in mlo2 mutants.

The mlo2 mutant requires components of non-host resistance and salicylic acid (SA) signalling for complete immunity against powdery mildew disease (Consonni et al. 
359

360

361

362

363

364

365

366

367

368

369

370

371

372

373

374

375

376

377

2006). Thus, the role of these components and the stress hormone SA in the mlo2 ROS phenotype was also tested using genetic epistasis analysis. Mutations in the components of non-host resistance, PENETRATION1 (PEN1; Collins et al. 2003), PEN2 (Lipka et al. 2005) and PEN3 (Bednarek et al. 2009) as single mutants had no effect on $\mathrm{O}_{3}$ sensitivity and each slightly enhanced ion leakage in the mlo2 mutant context; however, this increase was not statistically significant (Figure 4c). The role of SA was assessed with salicylic acid induction deficient2 (sid2-1) and phytoalexin deficient4 (pad4-4), which are SA biosynthesis (Wildermuth et al. 2001) and signalling mutants (Zhou et al. 1998), respectively. The enhanced $\mathrm{O}_{3}$ tolerance of $\mathrm{mlo} 2$ was lost in both mlo2-6 sid2-1 and mlo2-6 pad4-1 (Figure 4d and S5), indicating SA is required in the ROS signalling pathway regulated by MLO2.

To investigate further the role of ROS signalling, additional mlo2 double mutants involved in ROS and defence signalling were created and tested. These included double mutants with the $\mathrm{NAD}(\mathrm{P}) \mathrm{H}$ oxidase, respiratory burst oxidase homolog $\mathrm{C}$ (rbohC; rhd2-4 transposable element insertion allele; Foreman et al. 2003), the ROS signalling MAP kinases, mitogen activated kinase3 (mpk3-1) and mpk6-2 (Ahlfors et al. 2004b), the ABA biosynthesis mutant abscisic acid deficient3 (aba3-1; Xiong et al. 2001), the kinase mutant oxidative signal-inducible1 (oxi1-2; Rentel et al. 2004), and the co-receptor mutant, bri1-associated receptor kinase1-4 (bak1-4; Li et al. 2002). The rbohC, oxi1-2, mpk6-2, aba3-1 and bak1-4 mutations supressed the enhanced $\mathrm{O}_{3}$ tolerance of mlo2-6; mlo2-6 rbohC, mlo2-6 oxi1-2, mlo2-6 mpk6-2, mlo2-6 aba3-1 and mlo2-6 bak1-4 exhibited equal or greater $\mathrm{O}_{3}$-induced electrolyte leakage than Col-0 wild-type (Figure 4e and f). The aba3-1 mutant is $\mathrm{O}_{3}$-sensitive with higher electrolyte leakage than Col-0 wild-type ( 10\% vs. 5\%). The mlo2-6 aba3-1 mutant exhibited full $\mathrm{O}_{3}$ sensitivity with electrolyte leakage levels similar to the aba3-1 mutant $(\sim 12 \%)$. 
384 Unaltered mlo2-conditioned $\mathrm{O}_{3}$ tolerance was seen in mlo2-6 mpk3-1 (Figure 4e), 385 indicating that mutations in MPK3 do not affect the mlo2-6 phenotype.

\section{Powdery mildew infection and spontaneous callose deposition phenotypes of} double mutants harbouring $\mathrm{mlo2}$

The newly generated mlo2 double mutants described in the previous sections and their respective single mutants were then assayed for powdery mildew (Golovinomyces orontii) infection phenotypes. However, none of the tested single or double mutants had any statistically significant difference in $\underline{G}$. orontii host cell entry to their respective controls, which were Col-0 ( 90\% host cell entry) for the single mutants and mlo2-6 ( $50 \%$ host cell entry) for the double mutants (Figure S6). These results indicate that these loci are not involved in pre-invasive basal defence or mlo2-conditioned powdery mildew resistance.

In addition to enhanced resistance against powdery mildew attack, the mlo2 mutant exhibits developmentally controlled premature leaf senescence, which is preceded by spontaneous ectopic callose deposition in rosette leaves (Consonni et al. 2006; Consonni et al. 2010). Since in our conditions callose deposition, visualized microscopically in aniline blue-stained samples, was the more robust marker, we assayed the same set of single and double mutants for this phenotype in a time-course experiment. Rosette leaf samples were collected during weeks five to eight, i.e. timepoints before (five weeks), during (six-seven weeks) and after (eight weeks) full development of the callose deposition phenotype in mlo2 genotypes. Following aniline blue staining, these specimens were assessed semi-quantitatively for the occurrence of callose deposits. Similar to the mlo2-6 mutant, the aba3-1 and in particular the vtc11 single mutant showed early spontaneous callose deposition (Figure 5), while all other 
408 single mutants (bak1-4, mpk3-1, mpk6-2, oxi1-2, rbohC, rcd1-4, re-8 and ost1-3) had 409 phenotypes similar to Col-0 wild-type during the time-course (Figure 5a). The callose 410 deposition phenotypes of aba3-1 and vtc1-1 were additive with that of mlo2-6, resulting 411 in earlier and more pronounced callose deposits in mlo2-6 aba3-1 and mlo2-6 vtc1-1 412 double mutants (Figure 5). Remarkably, the rcd1-4 mutant completely suppressed the 413 mlo2-6 callose phenotype, with the mlo2-6 rcd1-4 double mutant behaving 414 indistinguishable from Col-0 wild-type, even in eight-week-old plants (Figure 5b and c). 415 All other mlo2-6 double mutants showed strong and consistent callose deposition 416 (Figure 5b). In summary, while mutants involved in ROS and defence signalling did not 417 impact the powdery mildew resistance phenotype of the mlo2-6 mutant, some of the 418 respective mutants either phenocopied/enhanced (aba3-1, vtc1-1) or fully suppressed 419 (rcd1-4) the spontaneous callose deposition phenotype of the mlo2-6 mutant.

Deregulated genes in the mlo2 mlo6 mlo12 triple mutant comprise abiotic stress response genes

422 To identify and further analyse genes that are deregulated in a mlo mutant context we mined publicly available gene expression data of the mlo2-6 mlo6-2 mlo12-1 mutant utilising lists of genes (Table S2a) with altered expression in the triple mutant at the age of five, six, and seven weeks previously identified by Consonni and co-workers (Consonni et al. 2010). Enriched gene ontology (GO) terms were identified among the selected genes (Table S2). Consistent with known phenotypes of this mutant, most enriched GO terms are related to immunity, senescence, biotic stress signalling, cell death, and antimicrobial compound synthesis (Table S2b, c, d). This confirms in part 430 the results of an earlier study (Consonni et al. 2010), however, we also found novel 431 gene expression patterns in this dataset that are related to abiotic stress and not 432 previously discussed. The GO classification "response to ROS" (GO: 0000302) was 
433 enriched in the six week gene set, while "response to hydrogen peroxide" (GO: 434 0042542), "response to ROS" (GO:0000302), and "response to oxidative stress" (GO: 435 0006979) were enriched in the seven week gene set. This pattern underscores the 436 involvement of ROS in processes regulated by MLO2, MLO6, and MLO12 and 437 suggests that ROS-signalling may be pre-activated during development in the mlo2-5, 438 mlo6-2 mlo12-1 triple mutant.

439 Unexpectedly, some GO classes related to abiotic stress response were also enriched; 440 these included "cold acclimation" (GO: 0009631), "response to abiotic stimulus" (GO: 4410009628 - see downregulated genes at seven weeks; Table S2f), and "response to 442 ABA" (GO: 0009737, at seven weeks). Four out of five genes comprising the genes 443 enriched for GO: 0009631 "cold acclimation" are known targets of the ABA444 independent abiotic stress signalling pathway regulated by the transcription factor 445 DREB2a (Table S2; Sakuma et al. 2006), prompting further examination of a potential 446 overlap between MLO-dependent and DREB2a-regulated gene sets. Respectively, 19, 44760 , and 33 genes were found in common between datasets for a transgenic line 448 overexpressing constitutively active DREB2A and the five, six and seven week 449 datasets of the mlo2-5 mlo6-2 mlo12-1 triple mutant (Table S2g). Together, these 450 represent a significant enrichment for DREB2a-regulated genes (Table S2h) among 451 the clade V MLO-regulated genes. This finding suggests that there is overlap between 452 DREB2a-associated signalling and the pathways downstream of these MLOs.

453 To further explore MLO-regulated genes, we analysed the hierarchical clustering of 454 expression profiles for MLO2-, MLO6- and MLO12-regulated genes across a collection 455 of publicly available transcript profiling experiments related to ROS, abiotic stress, 456 biotic stress, and hormone signalling. In total, these MLOs regulate 1646 genes during 457 leaf rosette development (Consonni et al. 2010). To facilitate analysis, we reduced the 
458

459

460

461

462

463

464

465

466

467

468

469

470

471

472

473

474

475

476

477

478

479

480

481

gene number by selecting, from the week six gene set, 106 genes with a fold induction of $>6$ in at least one experiment and show below that these are a representative set of MLO-regulated genes. The highest expression levels of these genes were seen in experiments related to abiotic stress, such as salt and osmotic stresses, and processes involving ROS production, such as biotic stress treatments (PAMPs, Botrytis, virulent and avirulent bacterial pathogens; Figure 6). Notably, $\mathrm{O}_{3}$, paraquat (methyl viologen) and $\mathrm{H}_{2} \mathrm{O}_{2}$ treatment also induced the expression of MLO-regulated genes. Generally, genes upregulated in the mlo triple mutant were also upregulated by stress treatments and those downregulated in the mlo triple mutant were likewise downregulated by stress. However, there is a block of experiments that exhibit predominately reduced expression of all MLO2 regulated genes; these are high light treatments, mitochondrial stress (rotenone treatment), the constitutive triple response1 (ctr1) mutant, and the rcd1-4 mutant (Figure 6).

The week six data used here has previously been used as a representative dataset for MLO-regulated genes (Consonni et al. 2010). To test how representative our results are, we utilized all genes from each of the three time points separately (Figures S7S9). Comparison of results from week five, six and seven demonstrates that, although these gene sets are largely distinct, all MLO-regulated genes exhibited similar expression behaviour across the selected experiments. Together these findings suggest that these MLOs act in the regulation of abiotic stress-dependent gene expression.

\section{MLO2 transcript accumulation is ROS-responsive}

To find out if MLO2 gene expression is ROS-responsive, publicly available microarray data from $\mathrm{O}_{3}$-exposed plants (Blomster et al. 2011) was mined. MLO2 transcript 
482 accumulation was found to be significantly ( 3-fold; $\mathrm{P}=0.03$ ) elevated in $\mathrm{O}_{3}$-treated 483 samples (Figure S3a). To experimentally verify this result, we assessed MLO2 484 transcript accumulation by quantitative reverse transcription polymerase chain reaction 485 (qRT-PCR) based on leaf samples that were exposed for $1 \mathrm{~h}$ to either $\mathrm{O}_{3}\left(300 \mathrm{nl} \mathrm{I}^{-1}\right)$ or 486 "clean air" (ambient $\mathrm{O}_{3}$ concentrations) as control. We confirmed that the MLO2 487 transcript accumulates to significantly ( 3-fold) higher levels in the $\mathrm{O}_{3}$-treated Col-0 488 samples (Figure S3b). Furthermore, MLO2 transcript accumulation was slightly, but 489 significantly, higher in the rcd1-4 mutant, which is hypersensitive to $\mathrm{O}_{3}$ and exhibits 490 enhanced ROS accumulation. Thus, we conclude that MLO2 is responsive to 491 extracellular ROS. 


\section{DISCUSSION}

494

495

496

497

498

499

500

501

502

503

504

505

506

507

508

509

510

511

512

513

514 In double mutant contexts, loss of MLO2 function conferred enhanced tolerance to a 515 variety of ROS-sensitive mutants representing various mechanisms of sensitivity 516 (Figure 4). These include ost1-3, which has more open stomata; vtc1-1, which is

\section{MLO2 negatively regulates ROS-induced cell death}

$\mathrm{O}_{3}$-tolerance in the mlo2 mutant suggests that MLO2 modulates ROS responses. Relative to known $\mathrm{O}_{3}$-tolerant genotypes, the mlo2 mlo6 mlo12 triple mutant counts among the most tolerant. Although many $\mathrm{O}_{3}$-sensitive mutants have been studied (reviewed in Vainonen et al. 2015), few $\mathrm{O}_{3}$-tolerant genotypes are known (Joo et al. 2005; Brosché et al. 2010; Booker et al. 2004; Overmyer et al. 2000). Interestingly, these include gpa1, which is defective in the heterotrimeric $\mathrm{G}$ protein a subunit, while the $\mathrm{G} \beta$ subunit mutant (agb1) shows enhanced $\mathrm{O}_{3}$ sensitivity (Joo et al. 2005; Booker et al. 2004). Antagonistic activity of MLO2 and AGB1 has been previously noted in relation to powdery mildew immunity (Lorek et al. 2013).

Altered ROS responses in leaves is common to MLO2, MLO6, and MLO12, but not to other MLOs (Figure 1a-c; Figure S1a). These clade V MLOs were previously shown to regulate powdery mildew immunity with unequal genetic redundancy (Consonni et al. 2010). Here, similar redundancy in the response to ROS was shown (Figure 1d). Specifically, the pattern of ROS tolerance in these mutants, i.e. partial ROS tolerance in mlo2, slight sensitivity or no phenotype in mlo6 and mlo12, and strong tolerance in the triple mutant, indicates unequal genetic redundancy of $M L O$ functions for this trait. The shared genetic architecture underlying the regulation of ROS and immune responses suggests mechanistic overlap between the physiological programs governing ROS tolerance and antifungal immunity. 
517 deficient in ascorbate and thus ROS scavenging; and re-8, which has an altered 518 chloroplast redox response. MLO2's involvement in many ROS-related processes 519 suggests it acts to dampen ROS signals in general, likely high up in the ROS signalling 520 cascade.

$521 \mathrm{O}_{3}$ enters leaves through open stomata and forms ROS in the apoplast; thus $\mathrm{O}_{3}$ 522 tolerance may be explained by altered stomatal responses. We comprehensively 523 addressed this by measuring the following, steady-state stomatal conductance by two 524 methods, the diurnal pattern of stomatal movements, water loss from detached leaves, 525 and $\mathrm{O}_{3}$-induced stomatal closure. All assays indicate wild-type-like phenotypes in $\mathrm{mlo}$ 526 mutants (Figures 3a-c and S4c). Also the photosynthesis rate was assessed; as 527 previously shown by chlorophyll fluorescence (Consonni et al. 2010), mlo2 mutants 528 exhibited wild-type photosynthesis rates (Figure S4A). Stomata in ost1 are 529 constitutively open; thus unchanged water loss and stomatal conductance but 530 enhanced $\mathrm{O}_{3}$-tolerance in the ost1 $\mathrm{mlo} 2$ double mutant provides further genetic 531 evidence that mlo2-mediated ROS tolerance is independent of stomata (Figure 3b). 532 Finally, infiltration with an in vitro XXO superoxide generating system, which delivers 533 ROS in a non-stomatal-dependent manner, further demonstrates this point (Figure 2a).

534 Together, these results indicate that the MLO2 regulates tolerance to extracellular 535 ROS independent of stomatal regulation. Meta-analysis of MLO2-regulated genes 536 (Table S2c, d) demonstrates an enrichment among upregulated genes for GO classes 537 related to $\mathrm{ROS}$ signalling. This indicates that the mlo2 mutant is primed for $\mathrm{O}_{3}$ 538 responsiveness and thus $\mathrm{MLO} 2$ operates as a negative regulator of ROS-induced 539 gene expression. This inference implies that MLO2 modulates the threshold for ROS540 induced cell death. We therefore conclude that MLO2 is a regulator of ROS responses, 541 including cell death. This finding is consistent with the previous notion that barley Mlo 
542 acts as a negative regulator of localized ROS production at fungal attack sites and 543 mesophyll cell death (Piffanelli et al. 2002).

\section{MLO2 functions in ROS signalling and immunity can be genetically uncoupled}

545 Epistasis analysis indicated that ROS tolerance conditioned by loss of MLO2 function 546 requires several known mediators of ROS, stress, and immune signalling. These 547 results further support the role of MLO2 as a regulator of stress-induced cell death and 548 allow dissection of the divergent processes regulated by MLO2. Consistent with the 549 role of ROS in immunity, some processes are needed for both mlo2-mediated 550 enhanced immunity against powdery mildew (Consonni et al. 2006) and enhanced 551 ROS tolerance (Figure 4). These include SA accumulation, and SA-dependent signal 552 transduction. By contrast, three PEN proteins are necessary for enhanced immunity 553 (Consonni et al. 2006), but not ROS tolerance (Figure 4c). These proteins function in 554 the synthesis and delivery of antimicrobial compounds; as such, they are not expected 555 to modulate ROS/cell death pathways.

556 Other processes required for enhanced ROS tolerance (Figure 4), but not enhanced 557 immunity (Figure S6), included stress responsive ABA production; ROS production 558 from RBOHC; the MAP kinase MPK6; and the BAK1 co-receptor, required for the 559 function of hormone and PAMP receptor complexes. These results suggest that MLO2 560 regulation of ROS signalling occurs upstream of at least two distinct signalling 561 pathways, which can be genetically uncoupled; one regulating immunity and the other 562 controlling cell death and other ROS processes. This suggests MLO2 is an integrator 563 of multiple signals, modulating various physiological responses (Figure 7).

564 The mlo2 mutant is immune against powdery mildew and appears to have pre565 activated ROS responses (Figure S6 and Table S2). This is in agreement with the 
566

567

568

569

570

571

572

573

574

575

576

577

578

579

580

581

582

583

584

585

586

587

588

589

590

previous observation that $\mathrm{O}_{3}$ can induce $\mathrm{SA}$ accumulation and immunity, for instance against the hemibiotrophic pathogen Pseudomonas syringae pv. maculicola in susceptible Arabidopsis and the biotrophic pathogen Blumeria graminis $f$. sp. tritici in susceptible wheat ( $\underline{\text { Triticum aestivum }}$ ) (Sharma et al. 1995; Pazarlar et al. 2017). Loss of MLO2 function also confers enhanced susceptibility to the hemibiotrophic pathogen Magnaporthe oryzae (Lyngkjær et al., 2000) and the necrotrophic pathogen Bipolaris sorokiniana (Kumar et al. 2001). Accordingly, $\mathrm{O}_{3}$ is a known enhancer of susceptibility to necrotrophic fungi (Manning and von Tiedemann, 1995). However, the mlo2 mutant is a notable exception to the general positive correlation between plant $\mathrm{O}_{3}$ sensitivity and susceptibility to necrotrophic pathogens (Manning and von Tiedemann, 1995) and the interaction between $\mathrm{O}_{3}$ and hemibiotrophic pathogens appears to be variable.

The original barley mlo mutant shares features with so-called lesion-mimic mutants, which ectopically trigger cell death, exhibit enhanced callose deposition, and confer enhanced immunity (Piffanelli et al. 2002). However, mlo-associated developmental cell death resembles premature senescence, as suggested by early pigment decay (Piffanelli et al. 2002). It remains unclear if enhanced cell death and callose deposition are required for immunity in barley mlo genotypes. Arabidopsis mlo2 and mlo2 mlo6 mlo12 mutants also exhibit the same phenotypes (Consonni et al. 2010). Transcriptomic studies of these mutants suggest the activation of a true senescence program (Consonni et al. 2010). MLO2 regulates resistance bearing many hallmarks of non-host penetration resistance (Humphry et al. 2006), which lacks cell death, further supporting that mlo-mediated resistance does not require cell death. Finally, the $\mathrm{O}_{3}$-sensitive $\mathrm{rcd} 1-4$ mutant completely supresses mlo2-associated spontaneous callose deposition, but had no effect on enhanced immunity (Figure 4 and S6). Thus, MLO2 may modulate the threshold for cell death activation in a ROS signalling pathway 
591 independent of processes related to immunity. These data further support previous 592 results demonstrating uncoupling of mlo2 phenotypes. Premature callose deposition 593 was separated from immunity in the mlo2 powdery mildew resistant 4 (pmr4) double 594 mutant (Consonni et al. 2010). PMR4 is a GLUCAN SYNTHASE-LIKE gene required 595 for wound- and pathogen-induced callose biosynthesis (Jacobs et al. 2003; Nishimura 596 et al. 2003). As previously noted, these results suggest the possibility of engineering 597 the favourable enhanced immunity of mlo2 genotypes without the undesirable 598 premature senescence (Consonni et al. 2010).

\section{MLO2 function is linked to abiotic stress responses}

600 Similar to mlo2, several $\mathrm{O}_{3}$-sensitive mutants (aba3-1, re-8, and vtc1-1) exhibited 601 enhanced callose deposition (Figure 5), underscoring the role of ROS in this process. 602 The aba3-1 mutant was of particular interest, as it suggests a role for abiotic stress 603 signalling pathways in this context. Analysis of MLO2-, MLO6-, MLO12-regulated 604 genes indicates enrichment of the GO category "cold acclimation", and targets of the 605 DREB2a transcription factor, indicating misregulation of abiotic stress marker genes 606 (Table S2b, e, h). DREB2 is a known RCD1-interaction partner (Jaspers et al. 2009).

607 DREB2A and RCD1 are involved in non-ABA-dependent abiotic stress signalling and 608 senescence, further implicating MLOs in abiotic stress. This connection suggests 609 targets of further research, which will be required to reveal the role of MLO2 in abiotic 610 stress response. Finally, these results in Arabidopsis further support the previously 611 suggested link of barley Mlo to abiotic stress responses (Piffanelli et al. 2002).

\section{A common role for MLO proteins as regulators of ROS responses?}

613 The biochemical function of MLO proteins remains unknown (Acevedo-Garcia et al. 614 2014; Kusch et al. 2016). Here we demonstrate that clade V MLOs act, in unequal 
615 genetic redundancy, as negative regulators of ROS signalling. This raises the intriguing possibility that involvement in ROS signalling could be a general feature of MLO proteins. ROS signalling has roles in various aspects of plant life, including immunity, senescence and abiotic stress responses, which are all associated with clade V MLO functions. Additionally, ROS regulate developmental processes governed by other Arabidopsis MLOs. In plant sexual reproduction, the pollen tube grows to, and bursts at, synergid cells of the female gametophyte to release its sperm cells and mediate dual fertilization. MLO7 and the receptor-like kinase, FERONIA (FER), act in pollen tube guidance and reception (Kessler et al. 2010). In mlo7 mutants, pollen tubes grow past the synergid cells, or arrive but fail to release the sperm cells. Remarkably, the fer mutant exhibits a powdery mildew resistance phenotype. ROS generated by an NADPH oxidase act in concert with a calcium gradient, both of which are essential for rapid polar pollen tube growth (Potocký et al. 2007; Li et al. 1999). Notably, FER was recently shown to regulate high-level ROS accumulation, near the entrance to the female gametophyte, which is required to induce calcium-dependent pollen tube rupture and sperm release (Duan et al. 2014).

MLO4 and MLO11 are involved in modulating root thigmomorphogenesis. In mlo4 and mlo11 mutants, roots grow in a tightly curled pattern; this anisotropic chiral root expansion phenotype requires polar auxin transport and calcium signalling (Bidzinski et al. 2014; Chen et al. 2009). Mechanical stimulation of Arabidopsis roots results in a

635 specific signature of calcium transients that further triggers apoplastic alkalinisation and $R B O H C$-dependent ROS accumulation, transmitting touch stimuli into appropriate growth responses (Monshausen et al. 2009).

638 Taken together, MLO function is linked to ROS responses in a variety of physiological 639 processes. However, the connection between MLO function and ROS signalling can 
640 be in part genetically uncoupled. It remains to be discovered whether this interplay is 641 indeed tied to the core biochemical activity of MLO proteins and if so, how it is 642 established at the mechanistic level.

643 


\section{ACKNOWLEDGEMENTS}

645 We thank Tuomas Puukko, Leena Grönholm, Airi Lamminmäki, and Jelena Odintsova 646 for excellent technical support. NASC (www.arabidopsis.info) is acknowledged for 647 providing Arabidopsis seeds. This work was supported by the following grants: 648 University of Helsinki three year research allocation to KO; Academy of Finland 649 Fellowship (decisions no. 251397, 256073 and 283254) to KO; Academy of Finland 650 Center of Excellence in Primary Producers 2014-2019 (decisions \#271832 and 651 307335); and a Finnish Cultural Foundation grant to FC. FC and OM are members of 652 the University of Helsinki Doctoral Program in Plant Science (DPPS). HW was 653 supported by a PhD fellowship from the Chinese Scholarship Council (CSC). RK was 654 supported by the Staff Mobility program of the EU-India BRAVE Consortium. 655 Computing resources provided by the Finnish IT Center for Science (CSC; www.csc.fi) 656 are gratefully acknowledged. 


\section{REFERENCES}

659

660

661

662

663

664

665

666

667

668

669

670

671

672

673

674

675

676

677

678

679

680

681 682 in mutants of Arabidopsis thaliana MILDEW LOCUS O genes. Plant, Cell \& Environment $683 \quad 37,2738-2753$.

Acevedo-Garcia J., Gruner K., Reinstädler A., Kemen A., Kemen E., Cao L., .., Panstruga R. (2017) The powdery mildew-resistant Arabidopsis mlo2 mlo6 mlo12 triple mutant displays altered infection phenotypes with diverse types of phytopathogens. Scientific Reports 7, 27.

Acevedo-Garcia J., Kusch S. \& Panstruga R. (2014) Magical mystery tour - MLO proteins in plant immunity and beyond. New Phytologist 204, 273-281.

Adam L. \& Somerville S.C. (1996) Genetic characterization of five powdery mildew disease resistance loci in Arabidopsis thaliana. Plant Journal 9, 341-356.

Ahlfors R., Lång S., Overmyer K., Jaspers P., Brosché M., Tauriainen A., ..., Kangasjärvi J. (2004a) Arabidopsis RADICAL-INDUCED CELL DEATH1 belongs to the WWE proteinprotein interaction domain protein family and modulates abscisic acid, ethylene, and methyl jasmonate responses. Plant Cell 16, 1925-1937.

Ahlfors R., Macioszek V., Rudd J., Brosché M., Schlichting R., Scheel D. \& Kangasjärvi J. (2004b) Stress hormone-independent activation and nuclear translocation of mitogenactivated protein kinases in Arabidopsis thaliana during ozone exposure. Plant Journal 40, $512-522$.

Alonso J.M., Hirayama T., Roman G., Nourizadeh S. \& Ecker J.R. (1999) EIN2, a bifunctional transducer of ethylene and stress responses in Arabidopsis. Science 284, 2148-2152.

Bednarek P., Piślewska-Bednarek M., Svatoš A., Schneider B., Doubský J., Mansurova M., ..., Schulze-Lefert P. (2009) A glucosinolate metabolism pathway in living plant cells mediates broad-spectrum antifungal defense. Science 323, 101-106.

Bidzinski P., Noir S., Shahi S., Reinstädler A., Gratkowska D.M. \& Panstruga R. (2014)

81 Physiological characterization and genetic modifiers of aberrant root thigmomorphogenesis 
684 Blomster T., Salojärvi J., Sipari N., Brosché M., Ahlfors R., Keinänen M., Overmyer K. \& 685 Kangasjärvi J. (2011) Apoplastic reactive oxygen species transiently decrease auxin 686 signaling and cause stress-induced morphogenic response in Arabidopsis. Plant 687 Physiology 157, 1866-1883.

688 Booker F.L., Burkey K.O., Overmyer K. \& Jones A.M. (2004) Differential responses of G689 protein Arabidopsis thaliana mutants to ozone. New Phytologist 162, 633-641.

690 Bretz F., Hothorn T. \& Westfall P.H. (2011) Multiple comparisons using R. CRC Press, Boca 691 Raton.

692 Brosché M., Blomster T., Salojärvi J., Cui F., Sipari N., Leppälä J., ..., Kangasjärvi J. (2014)

693 Transcriptomics and functional genomics of ROS-induced cell death regulation by 694 RADICAL-INDUCED CELL DEATH1. PLoS Genetics 10, e1004112.

695 Brosché M., Merilo E., Mayer F., Pechter P., Puzõrjova I., Brader G., Kangasjärvi J. \& Kollist 696 H. (2010) Natural variation in ozone sensitivity among Arabidopsis thaliana accessions and 697 its relation to stomatal conductance. Plant, Cell \& Environment 33, 914-925.

698 Büschges R., Hollricher K., Panstruga R., Simons G., Wolter M., Frijters A., ..., Schulze699 Lefert P. (1997) The barley Mlo gene: A novel control element of plant pathogen $700 \quad$ resistance. Cell 88, 695-705.

701 Chen Z.Y., Noir S., Kwaaitaal M., Hartmann H.A., Wu M.J., Mudgil Y., .., Jones A.M. (2009) 702 Two seven-transmembrane domain MILDEW RESISTANCE LOCUS O proteins cofunction 703 in Arabidopsis root thigmomorphogenesis. Plant Cell 21, 1972-1991.

704 Collins N.C., Thordal-Christensen H., Lipka V., Bau S., Kombrink E., Qiu J.L., ..., Schulze705 Lefert P. (2003) SNARE-protein-mediated disease resistance at the plant cell wall. Nature $706 \quad 425,973-977$.

707 Conklin P.L., Pallanca J.E., Last R.L. \& Smirnoff N. (1997) L-ascorbic acid metabolism in the 708 ascorbate-deficient Arabidopsis mutant vtc1. Plant Physiology 115, 1277-1285.

709 Consonni C., Bednarek P., Humphry M., Francocci F., Ferrari S., Harzen A., van Themaat 710 E.V.L. \& Panstruga R. (2010) Tryptophan-derived metabolites are required for antifungal 711 defense in the Arabidopsis mlo2 mutant. Plant Physiology 152, 1544-1561. 
712 Consonni C., Humphry M.E., Hartmann H.A., Livaja M., Durner J., Westphal L., ...,

713 Panstruga R. (2006) Conserved requirement for a plant host cell protein in powdery

714 mildew pathogenesis. Nature Genetics 38, 716-720.

715 Devoto A., Hartmann H.A., Piffanelli P., Elliott C., Simmons C., Taramino G., ..., Panstruga

716 R. (2003) Molecular phylogeny and evolution of the plant-specific seven-transmembrane

717 MLO family. Journal of Molecular Evolution 56, 77-88.

718 Duan Q., Kita D., Johnson E.A., Aggarwal M., Gates L., Wu H.-M. \& Cheung A.Y. (2014)

719 Reactive oxygen species mediate pollen tube rupture to release sperm for fertilization in

720 Arabidopsis. Nature Communications 5, 3129.

721 Ellinger D., Naumann M., Falter C., Zwikowics C., Jamrow T., Manisseri C., Somerville S.C.

722 \& Voigt C.A. (2013) Elevated early callose deposition results in complete penetration

723 resistance to powdery mildew in Arabidopsis. Plant Physiology 161, 1433-1444.

724 Ellis C. \& Turner J.G. (2002) A conditionally fertile coi1 allele indicates cross-talk between 725 plant hormone signalling pathways in Arabidopsis thaliana seeds and young seedlings.

$726 \quad$ Planta 215, 549-556.

727 Foreman J., Demidchik V., Bothwell J.H.F., Mylona P., Miedema H., Torres M.A., ..., Dolan

728 L. (2003) Reactive oxygen species produced by NADPH oxidase regulate plant cell

729 growth. Nature 422, 442-446.

730 Freisleben R. \& Lein A. (1942) Über die Auffindung einer mehltauresistenten Mutante nach

731 Röntgenbestrahlung einer anfälligen reinen Linie von Sommergerste. Naturwissenschaften

732 30, 608.Gadjev I., Vanderauwera S., Gechev T.S., Laloi C., Minkov I.N., Shulaev V., ...,

733 van Breusegem F. (2006) Transcriptomic footprints disclose specificity of reactive oxygen

734 species signaling in Arabidopsis. Plant Physiology 141, 436-445.

735 Gapper C. \& Dolan L. (2006) Control of plant development by reactive oxygen species. Plant

$736 \quad$ Physiology 141, 341-345.

737 Georgii E., Salojärvi J., Brosché M., Kangasjärvi J. \& Kaski S. (2012) Targeted retrieval of 738 gene expression measurements using regulatory models. Bioinformatics 28, 2349-2356. 
Hellemans J., Mortier G., Paepe A. de, Speleman F. \& Vandesompele J. (2007) qBase relative quantification framework and software for management and automated analysis of real-time quantitative PCR data. Genome Biology 8, R19.

742

Hückelhoven R., Dechert C. \& Kogel K.H. (2001) Non-host resistance of barley is associated with a hydrogen peroxide burst at sites of attempted penetration by wheat powdery mildew fungus. Molecular Plant Pathology 2, 199-205.

Humphry M., Consonni C. \& Panstruga R. (2006) mlo-based powdery mildew immunity. Silver bullet or simply non-host resistance? Molecular Plant Pathology 7, 605-610.

Huysmans M., Lema A S., Coll N.S. \& Nowack M.K. (2017) Dying two deaths - programmed cell death regulation in development and disease. Current Opinion in Plant Biology 35, 3744.

Jabs T., Dietrich R.A. \& Dangl J.L. (1996) Initiation of runaway cell death in an Arabidopsis mutant by extracellular superoxide. Science 273, 1853-1856.

Jacobs A.K., Lipka V., Burton R.A., Panstruga R., Strizhov N., Schulze-Lefert P. \& Fincher G.B. (2003) An Arabidopsis callose synthase, GSL5, is required for wound and papillary callose formation. Plant Cell 15, 2503-2513.

Jaspers P., Blomster T., Brosché M., Salojärvi J., Ahlfors R., Vainonen J.P., ..., Kangasjärvi J. (2009) Unequally redundant RCD1 and SRO1 mediate stress and developmental responses and interact with transcription factors. Plant Journal 60, 268-279.

Jones J.D.G. \& Dangl J.L. (2006) The plant immune system. Nature 444, 323-329.

Joo J.H., Wang S., Chen J.G., Jones A.M. \& Fedoroff N.V. (2005) Different signaling and cell death roles of heterotrimeric $\mathrm{G}$ protein alpha and beta subunits in the Arabidopsis oxidative stress response to ozone. Plant Cell 17, 957-970.

Kessler S.A., Shimosato-Asano H., Keinath N.F., Wuest S.E., Ingram G., Panstruga R. \& Grossniklaus U. (2010) Conserved molecular components for pollen tube reception and fungal invasion. Science 330, 968-971. 
Kollist T., Moldau H., Rasulov B., Oja V., Rämma H., Hüve K., ..., Kollist H. (2007) A novel device detects a rapid ozone-induced transient stomatal closure in intact Arabidopsis and its absence in abi2 mutant. Physiologia Plantarum 129, 796-803.

Kumar J., Hückelhoven R., Beckhove U., Nagarajan S. \& Kogel KH. (2001) A compromised Mlo pathway affects the response of barley to the necrotrophic fungus Bipolaris sorokiniana (teleomorph: Cochliobolus sativus) and its toxins. Phytopathology 91, 127133.

Kusch S., Pesch L. \& Panstruga R. (2016) Comprehensive phylogenetic analysis sheds light on the diversity and origin of the MLO family of integral membrane proteins. Genome Biology and Evolution 8, 878-895.

Li H., Lin Y., Heath R.M., Zhu M.X. \& Yang Z. (1999) Control of pollen tube tip growth by a Rop GTPase-dependent pathway that leads to tip-localized calcium influx. Plant Cell 11, $1731-1742$.

Li J., Wen J., Lease K.A., Doke J.T., Tax F.E. \& Walker J.C. (2002) BAK1, an Arabidopsis LRR receptor-like protein kinase, interacts with BRI1 and modulates brassinosteroid signaling. Cell 110, 213-222.

Lipka V., Dittgen J., Bednarek P., Bhat R., Wiermer M., Stein M., ..., Schulze-Lefert P. (2005) Pre- and postinvasion defenses both contribute to nonhost resistance in Arabidopsis. Science 310, 1180-1183.

Lorek J., Griebel T., Jones A.M., Kuhn H. \& Panstruga R. (2013) The role of Arabidopsis heterotrimeric G-protein subunits in MLO2 function and MAMP-triggered immunity. Molecular Plant-Microbe Interactions 26, 991-1003.

Lyngkjær M., Newton A., Atzema J. \& Baker S. (2000) The barley mlo-gene: an important powdery mildew resistance source. Agronomie, 20, 745-756.

Mellersh D.G., Foulds I.V., Higgins V.J. \& Heath M.C. (2002) $\mathrm{H}_{2} \mathrm{O}_{2}$ plays different roles in determining penetration failure in three diverse plant-fungal interactions. Plant Journal 29, 257-268. 
Monshausen G.B., Bibikova T.N., Weisenseel M.H. \& Gilroy S. (2009) Ca ${ }^{2+}$ regulates reactive oxygen species production and $\mathrm{pH}$ during mechanosensing in Arabidopsis roots. Plant Cell 21, 2341-2356.

Müller J., Piffanelli P., Devoto A., Miklis M., Elliott C., Ortmann B., Schulze-Lefert P. \& Panstruga R. (2005) Conserved ERAD-like quality control of a plant polytopic membrane protein. Plant Cell 17, 149-163.

Nishimura M.T., Stein M., Hou B.H., Vogel J.P., Edwards H. \& Somerville S.C. (2003) Loss of a callose synthase results in salicylic acid-dependent disease resistance. Science 301, 969-972.

Overmyer K., Kollist H., Tuominen H., Betz C., Langebartels C., Wingsle G., ..., Kangasjärvi J. (2008) Complex phenotypic profiles leading to ozone sensitivity in Arabidopsis thaliana mutants. Plant, Cell \& Environment 31, 1237-1249.

Overmyer K., Tuominen H., Kettunen R., Betz C., Langebartels C., Sandermann H. \& Kangasjärvi J. (2000) Ozone-sensitive Arabidopsis rcd1 mutant reveals opposite roles for ethylene and jasmonate signaling pathways in regulating superoxide-dependent cell death. Plant Cell 12, 1849-1862.

Overmyer K., Wrzaczek M. \& Kangasjärvi J. (2009) Reactive oxygen species in ozone toxicity. In Reactive oxygen species in plant signaling (eds. L. A. Rio \& A. Puppo), pp. 191-207. Springer Berlin Heidelberg, Berlin, Heidelberg.

Panstruga R. (2005) Serpentine plant MLO proteins as entry portals for powdery mildew fungi. Biochemical Society Transactions 33, 389-392.

Pazarlar S., Cetinkaya N., Bor M. \& Ozdemir F. (2017) Ozone triggers different defence mechanisms against powdery mildew (Blumeria graminis DC. Speer f. sp. tritici) in susceptible and resistant wheat genotypes. Functional Plant Biology, 44, 1016-1028.

Piffanelli P., Zhou F.S., Casais C., Orme J., Jarosch B., Schaffrath U., ..., Schulze-Lefert P. (2002) The barley MLO modulator of defense and cell death is responsive to biotic and abiotic stress stimuli. Plant Physiology 129, 1076-1085. 
819 Polyn S., Willems A. \& Veylder L. de (2015) Cell cycle entry, maintenance, and exit during 820 plant development. Current Opinion in Plant Biology 23, 1-7.

821 Potocký M., Jones M.A., Bezvoda R., Smirnoff N. \& Zárský V. (2007) Reactive oxygen 822 species produced by NADPH oxidase are involved in pollen tube growth. New Phytologist $823 \quad 174,742-751$.

824 Qi J., Wang J., Gong Z. \& Zhou J.-M. (2017) Apoplastic ROS signaling in plant immunity. 825 Current Opinion in Plant Biology 38, 92-100.

826 Rao M.V., Lee H.-i. \& Davis K.R. (2002) Ozone-induced ethylene production is dependent on 827 salicylic acid, and both salicylic acid and ethylene act in concert to regulate ozone-induced 828 cell death. Plant Journal 32, 447-456.

829 Rentel M.C., Lecourieux D., Ouaked F., Usher S.L., Petersen L., Okamoto H., .., Knight 830 M.R. (2004) OXI1 kinase is necessary for oxidative burst-mediated signalling in $831 \quad$ Arabidopsis. Nature 427, 858-861.

832 Rogers H. \& Munné-Bosch S. (2016) Production and scavenging of reactive oxygen species 833 and redox signaling during leaf and flower senescence: similar but different. Plant 834 Physiology 171, 1560-1568.

835 Saji S., Bathula S., Kubo A., Tamaoki M., Aono M., Sano T., ..., Saji H. (2017) Ozone836 sensitive Arabidopsis mutants with deficiencies in photorespiratory enzymes. Plant and 837 Cell Physiology 58, 914-924.

838 Saji S., Bathula S., Kubo A., Tamaoki M., Kanna M., Aono M., ..., Saji H. (2008) Disruption of 839 a gene encoding C4-dicarboxylate transporter-like protein increases ozone sensitivity 840 through deregulation of the stomatal response in Arabidopsis thaliana. Plant and Cell 841 Physiology 49, 2-10.

842 Sakuma Y., Maruyama K., Qin F., Osakabe Y., Shinozaki K. \& Yamaguchi-Shinozaki K. 843 (2006) Dual function of an Arabidopsis transcription factor DREB2A in water-stress844 responsive and heat-stress-responsive gene expression. Proceedings of the National 845 Academy of Sciences of the United States of America 103, 18822-18827. 
Sharma Y.K., Leon J., Raskin I, \& Davis K.R.(1995) Ozone-induced responses in Arabidopsis thaliana: The role of salicylic acid in the accumulation of defense-related transcripts and induced resistance. Proceedings of the National Academy of Sciences of the United States of America 93, 5099-5104,

Staswick P.E., Su W. \& Howell S.H. (1992) Methyl jasmonate inhibition of root growth and induction of a leaf protein are decreased in an Arabidopsis thaliana mutant. Proceedings of the National Academy of Sciences of the United States of America 89, 6837-6840.

Swanson S. \& Gilroy S. (2010) ROS in plant development. Physiologia Plantarum 138, 384854392.

855 Torres M.A. (2010) ROS in biotic interactions. Physiologia Plantarum 138, 414-429.

856 Tuteja N. (2007) Abscisic acid and abiotic stress signaling. Plant Signaling \& Behavior 2, $857 \quad 135-138$.

858 Vahisalu T., Puzõrjova I., Brosché M., Valk E., Lepiku M., Moldau H., .., Kollist H. (2010) 859 Ozone-triggered rapid stomatal response involves the production of reactive oxygen 860 species, and is controlled by SLAC1 and OST1. Plant Journal 62, 442-453.

861 Vainonen J.P. \& Kangasjärvi J. (2015) Plant signalling in acute ozone exposure. Plant, Cell \& 862 Environment 38, 240-252.

863 van Schie, C.C.N. \& Takken F.L.W. (2014) Susceptibility genes 101: how to be a good host. 864 Annual Review of Phytopathology 52, 551-581.

865 Voigt C.A. (2014) Callose-mediated resistance to pathogenic intruders in plant defense866 related papillae. Frontiers in Plant Science 5, 168.

867 Wildermuth M.C., Dewdney J., Wu G. \& Ausubel F.M. (2001) Isochorismate synthase is 868 required to synthesize salicylic acid for plant defence. Nature 414, 562-565.

869 Wohlgemuth H., Mittelstrass K., Kschieschan S., Bender J., Weigel H.-J., Overmyer K., ..., 870 Langebartels C. (2002) Activation of an oxidative burst is a general feature of sensitive 871 plants exposed to the air pollutant ozone. Plant, Cell \& Environment 25, 717-726. 
872 Wrzaczek M., Brosché M., Salojärvi J., Kangasjärvi S., Idänheimo N., Mersmann S., ...,

873 Kangasjärvi J. (2010) Transcriptional regulation of the CRK/DUF26 group of receptor-like

874 protein kinases by ozone and plant hormones in Arabidopsis. BMC Plant Biology 10, 95.

875 Xiong L., Ishitani M., Lee H. \& Zhu J.K. (2001) The Arabidopsis LOS5/ABA3 locus encodes a

876 molybdenum cofactor sulfurase and modulates cold stress- and osmotic stress-responsive

877 gene expression. Plant Cell 13, 2063-2083.

878 Yi X., Du Z. \& Su Z. (2013) PlantGSEA: a gene set enrichment analysis toolkit for plant

879 community. Nucleic Acids Research 41, W98-103.

880 Zhou N., Tootle T.L., Tsui F., Klessig D.F. \& Glazebrook J. (1998) PAD4 functions upstream

881 from salicylic acid to control defense responses in Arabidopsis. Plant Cell 10, 1021-1030.

882

883 


\section{FIGURE LEGENDS}

885 Figure 1: mlo2 mutants show enhanced $\mathrm{O}_{3}$ tolerance.

886 (a) Phenotype of Col-0, mlo2-5, mlo6-2, and mlo12-1 plants that had been exposed to $887 \mathrm{O}_{3}\left(350 \mathrm{nl} \mathrm{l}^{-1}\right)$ for $6 \mathrm{~h}$ and photographed at $48 \mathrm{~h}$ post begin of exposure. Arrows indicate 888 examples of $\mathrm{O}_{3}$-damaged leaves. Scale bar $=1 \mathrm{~cm}$. (b) Cell death assayed as 889 electrolyte leakage from plants shown in (a). (c) Electrolyte leakage from an $\mathrm{O}_{3}(0,250$, 890300,350 , and $400 \mathrm{nl} \mathrm{l}^{-1}$ ) dose-response experiment for Col-0, mlo2-5, mlo6-2, mlo12891 1, and the highly $\mathrm{O}_{3}$-sensitive radical-induced cell death1-4 (rcd1-4) mutant as a 892 control. (d) Electrolyte leakage upon high $\mathrm{O}_{3}$ concentration (400 and $500 \mathrm{nl} \mathrm{I}^{-1}$ ) 893 exposure, exceeding the tolerance threshold of mlo2-5, for Col-0, mlo2-5, mlo6-2, 894 mlo12-1 single mutants and the for the mlo2-5 mlo6-2 mlo12-1 triple mutant. All 895 experiments were performed 3-6 times in independent biological repeats; with one 896 representative experiment shown. All data presented are means \pm standard deviation $897(\mathrm{SD} ; \mathrm{n}=5)$ for electrolyte leakage expressed as \% of total ions determined after 898 disrupting leaves by boiling. Statistical significance $(P<0.05)$ tested by two-way 899 ANOVA with post-hoc analysis. In (b), letters indicate significance groups were 900 genotypes with different letters are significantly different and in c) and (d) asterisks 901 show significant difference from Col-0 for each ozone concentration. See also 902 Figure S1.

903

904 Figure 2: mlo2 mutants are more tolerant to extracellular, but not to intracellular 905 ROS.

906 (a) Electrolyte leakage from leaves of Col-0, mlo2-6, and mlo2-5 mlo6-2 mlo12-1 907 plants, treated in vitro with an enzymatic superoxide generating system comprised of 
$9081 \mathrm{mM}$ xanthine and 0.1 unit $\mathrm{ml}^{-1} \mathrm{XXO}$. Data are presented as means $\pm \mathrm{SD}(\mathrm{n}=5)$ for ion 909 leakage expressed as \% of total ions determined after disrupting leaves by boiling. (b) 910 Root growth assay to determine MV sensitivity of Col-0 as well as the mlo2-5 and mlo29115 mlo6-2 mlo12-1 mutants. The highly MV-tolerant rcd1-4 mutant was included as a 912 control. Root length was quantified using ImageJ (https://imagej.nih.gov/ij/) from 913 photographs of nine-day-old seedlings grown on $0.5 \times$ MS plates (with $1 \%$ sucrose) in 914 the presence or absence of $0.1 \mu \mathrm{M}$ MV. Results are presented as means \pm SD of the 915 ratio between MV-treated and control seedlings, expressed as a percentage (\% of 916 control). All experiments were performed three times in independent biological 917 replicates and letters show statistical significance groups $(\mathrm{P}<0.05)$. In (a) significance 918 tested by two-way ANOVA with post-hoc analysis and a single representative experiment is shown, in (b) results from all experiments were pooled and analysed 920 ( $\mathrm{n}=36$ in total) and post-hoc analysis was used by computing contrasts from linear models and subjecting the $\mathrm{P}$ values to single-step error correction.

Figure 3: mlo2 mutants have unaltered stomatal responses.

924 (a) Stomatal conductance of Col-0, mlo2-6, mlo2-5 mlo6-2 mlo12-1, open stomata1-3 925 (ost1-3), and mlo2-6 ost1-3, measured with a Delta-T Device porometer (b) Fast 926 stomatal closure response to $\mathrm{O}_{3}$ in wild-type Col-0 and mlo2-5 mutant plants. The time course of stomatal conductance (expressed in relative units) of three- to four-week-old 928 plants was recorded. At the time point indicated with an arrow, $400 \mathrm{nl} \mathrm{l}^{-1} \mathrm{O}_{3}$ was applied. 929 Data points represent means \pm standard error of the mean (SEM; $n=12)$. (c) Water loss 930 measured from excised leaves of Col-0, mlo2-6, mlo2-5 mlo6-2 mlo12-1, open 931 stomata1-1 (ost1-3), and mlo2-6 ost1-3. All experiments performed at least three times 
932 in independent biological repeats. Representative experiments (a, c) or pooled data 933 from all experiments (b) are presented. In (a) and (c), different letters indicate 934 statistically different groups based on two-way ANOVA with post-hoc analysis. See 935 also Figure S4.

936

937 Figure 4: $\mathrm{O}_{3}$ phenotypes of mlo2 double mutants.

938 In all experiments, plants were exposed to $6 \mathrm{~h}$ of $\mathrm{O}_{3}$ at the indicated concentrations 939 and electrolyte leakage was quantified as a proxy for cell death at $24 \mathrm{~h}$ post-exposure. 940 All experiments were repeated in at least four independent biological replicates, with 941 data from a representative experiment shown. All data presented are means \pm SD $942(n=5)$ for electrolyte (ion) leakage expressed as \% of total ions determined after 943 disrupting leaves by boiling. Significance groups are indicated with lowercase letters, 944 where genotypes with different letters show significant differences $(P<0.05)$ tested by 945 two-way ANOVA with post-hoc analysis. (a) $\mathrm{O}_{3}$-sensitive mutants, $r c d 1-4$, re-8, ost19463 , and vtc1-1 and their respective mlo2-6 double mutants exposed to $250 \mathrm{nl} \mathrm{I}^{-1} \mathrm{O}_{3}$. (b) 947 The $\mathrm{O}_{3}$-sensitive jasmonate signalling mutants, jar1-1 and coi1-16, the $\mathrm{O}_{3}$-tolerant 948 ethylene insensitive ein2-1 mutant, and their respective mlo2-5 or mlo2-11 double 949 mutants were exposed to $250 \mathrm{nl} \mathrm{I}^{-1} \mathrm{O}_{3}$. (c) The mildew susceptible mutants, pen1-1, 950 pen2-1, pen3-1, and their respective mlo2-5 or mlo2-6 double mutants were exposed 951 to $375 \mathrm{nl} \mathrm{I}^{-1} \mathrm{O}_{3}$. (d) The SA signalling defective mutants, sid2-1, pad4-1, and their 952 respective mlo2-5 or mlo2-6 double mutants were exposed to $325 \mathrm{nl} \mathrm{I}^{-1} \mathrm{O}_{3}$. (e) The 953 ROS signalling mutants, rbohc, mpk3-1, mpk6-2, and their respective mlo2-6 double 954 mutants were exposed to $325 \mathrm{nl} \mathrm{I}^{-1} \mathrm{O}_{3}$. (f) The ABA deficient aba3-1 mutant, the immune 955 and hormone signalling co-receptor mutant, bak1-4, the early ROS signalling mutant 
956

957

958

959

960

961

962

963

964

965

966

967

968

969

970

971

972

973

974

975

976

977

978

979

oxi1-2, and their respective mlo2-6 double mutants were exposed to $300 \mathrm{nl} \mathrm{I}^{-1} \mathrm{O}_{3}$. Data from three separate exposures are plotted together and separated by vertical lines on the graph; comparisons should only be made within each exposure, each of which has its own wild-type Col-0 control. See Figure $\mathrm{S} 5$ for photographs of $\mathrm{O}_{3}$-exposed plants of selected genotypes.

Figure 5: Some mlo2 double mutants exhibit altered spontaneous callose deposition.

(a) Semi-quantitative assay of callose deposition. Plants of the indicated genotypes at five-, six-, seven-, and eight-weeks of age were stained for callose visualization with analine blue and visually scored for the presence of callose deposits under an epifluorescence microscope. Semi-quantitative assessment of callose deposition was performed as described in Materials and Methods. The experiment was performed four times, with three to four samples per genotype and age, and data pooled. Shown are the mean $\pm S D$ of the four experiments. Asterisks indicate a statistically significant difference $(\mathrm{P}<0.05)$ compared to Col-0 (upper panel) or mlo2-6 (lower panel) based on post-hoc analysis by computing contrasts from linear models and subjecting the $P$ values to single-step error correction. (b) Representative photos of selected mutant phenotypes. Scale bar, $500 \mu \mathrm{m}$, is valid for all micrographs.

Figure 6: MLO genes regulate abiotic stress response genes.

(a) Hierarchical clustering analysis of expression profiles for MLO-regulated genes. We selected 106 representative MLO2-, MLO6-, MLO12-regulated genes from the week six gene set (Consonni et al. 2010) with a fold induction of >six in at least one experiment (see Table S2i for gene list) and subjected their expression profiles to 
980 hierarchical clustering analysis. A collection of publicly available transcript profiling 981 experiments related to ROS, abiotic stress, biotic stress, hormone signalling, and 982 transcriptional regulators was used (see Table S2i for list of experiment accession 983 numbers). The same analysis was performed with all MLO-regulated genes and the 984 week five, six, and seven data is presented in Supplemental Figures S7-S9, 985 respectively.

986 Figure 7: Model of MLO2 as an integrator of abiotic and biotic stress responses.

$987 \mathrm{O}_{3}$ enters leaves through open stomata, indicated by two guard cells $(\mathrm{GC})$, a process 988 that is not regulated by MLO2, and reacts in the apoplast to form other reactive oxygen 989 species (ROS). We hypothesize that MLO2 integrates various inputs from abiotic and 990 biotic stress sensors, such as extracellular ROS, e.g. derived from $\mathrm{O}_{3}$ and microbe991 associated molecular patterns (MAMPs) originating from pathogens. ROS signals are 992 perceived and transmitted into the cell (denoted by the box) by yet unknown 993 mechanisms. We suggest that MLO2 acts to attenuate a common ROS signalling 994 pathway that splits downstream to regulate various processes, such as tolerance to 995 extracellular ROS, spontaneous callose deposition, antifungal immunity and cell death. MLO2-regulated processes are inferred from mlo2 mutant phenotypes and the 997 divergent pathways from the different loci genetically modulating mlo2 mutant 998 phenotypes. Genes negatively contributing to a given pathway are indicated with * 999 Diagrams inside the box denote signalling events inside the plant cell without implying 1000 information on their subcellular localization. 
(a)

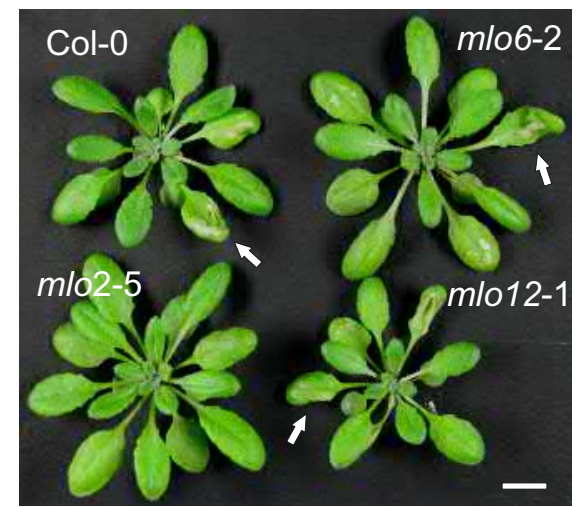

(b)
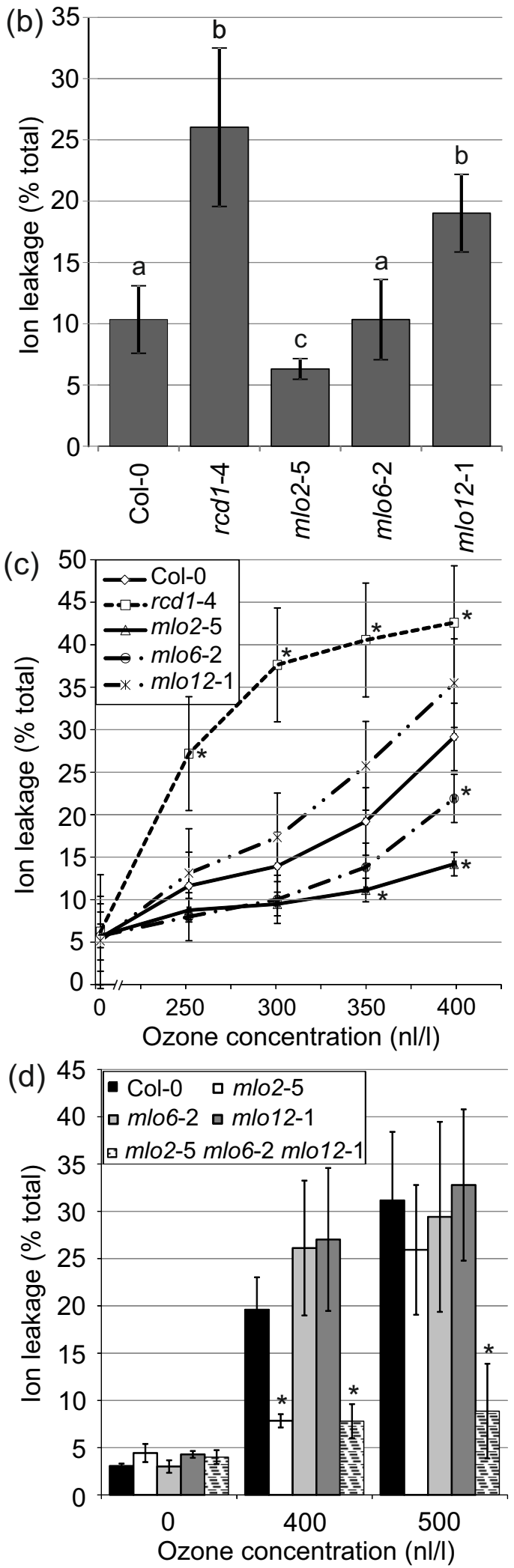

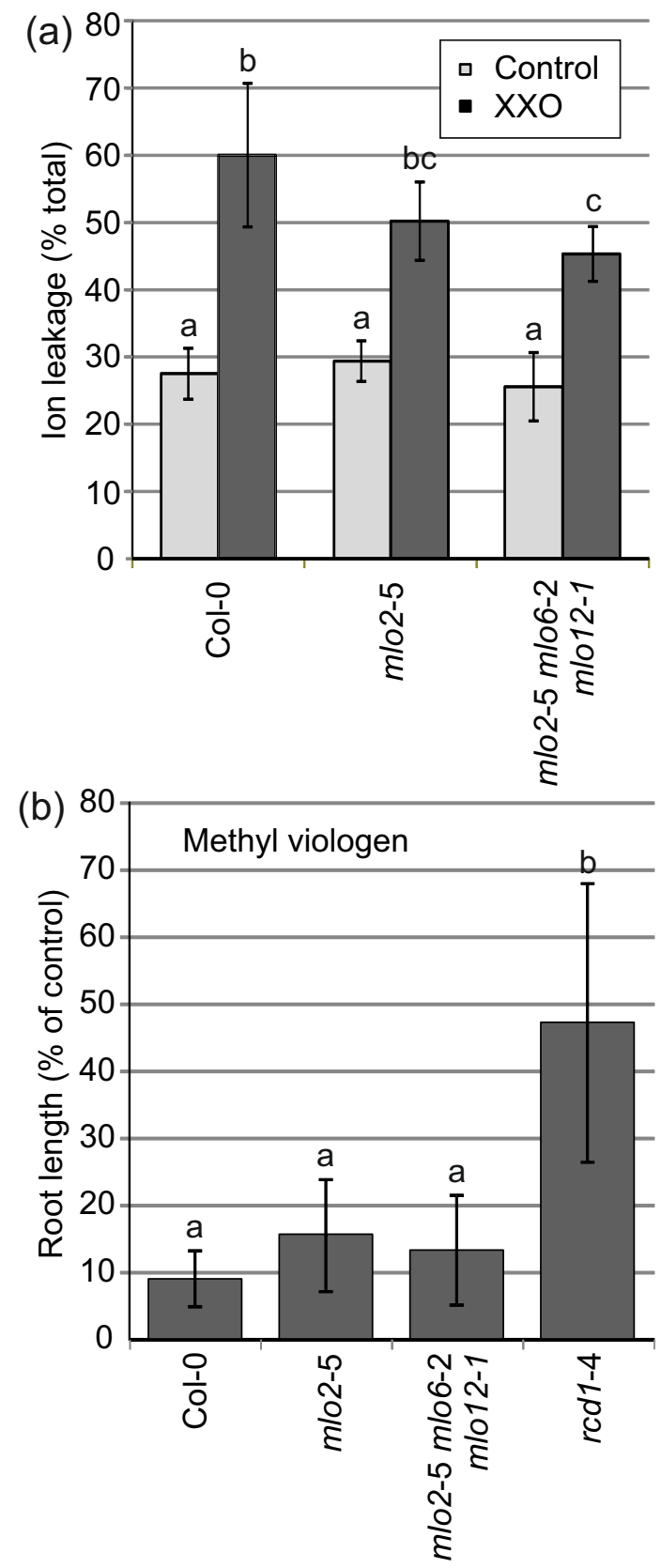

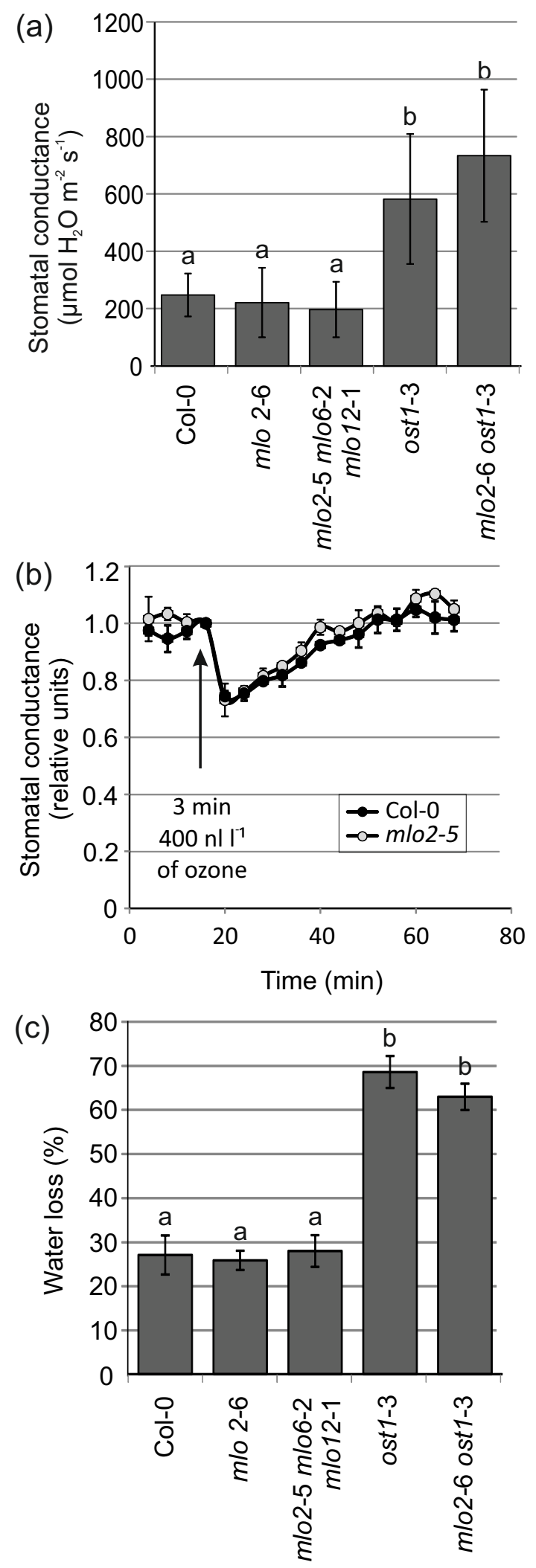

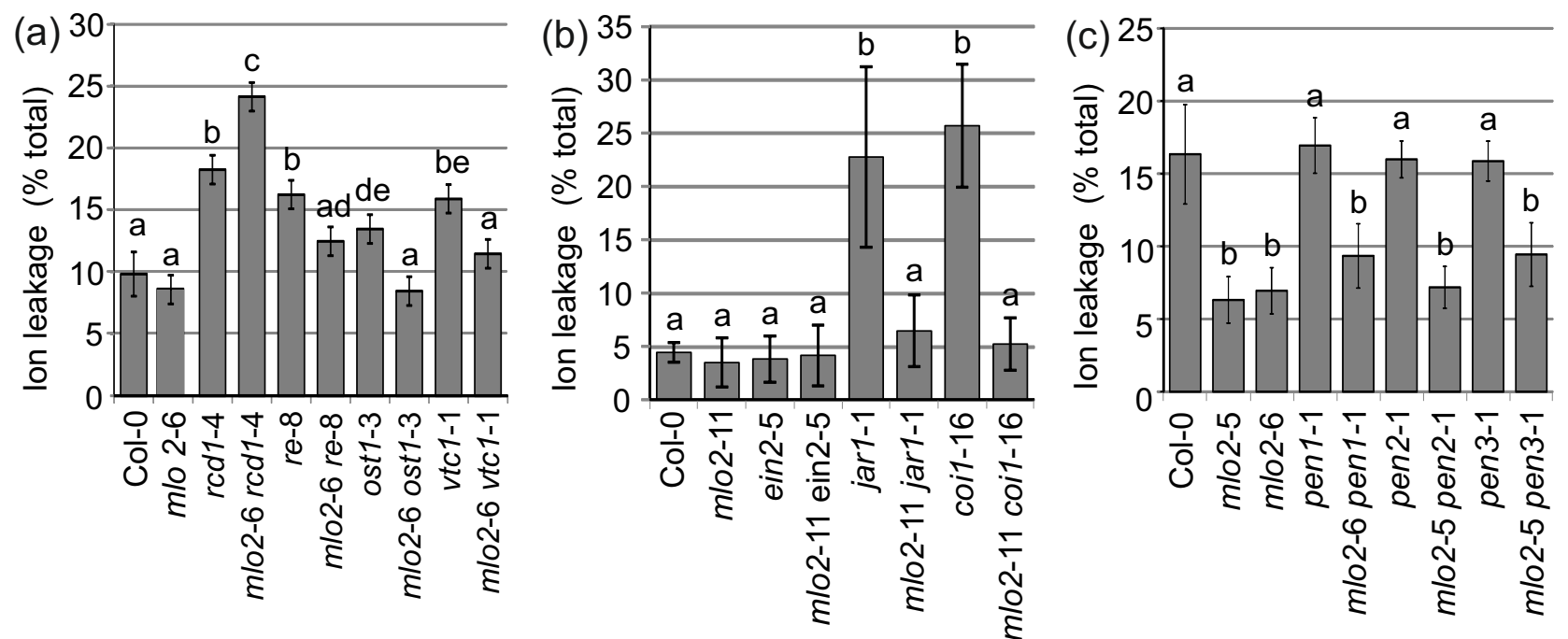

옹 눈

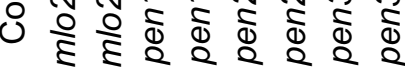

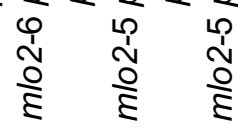
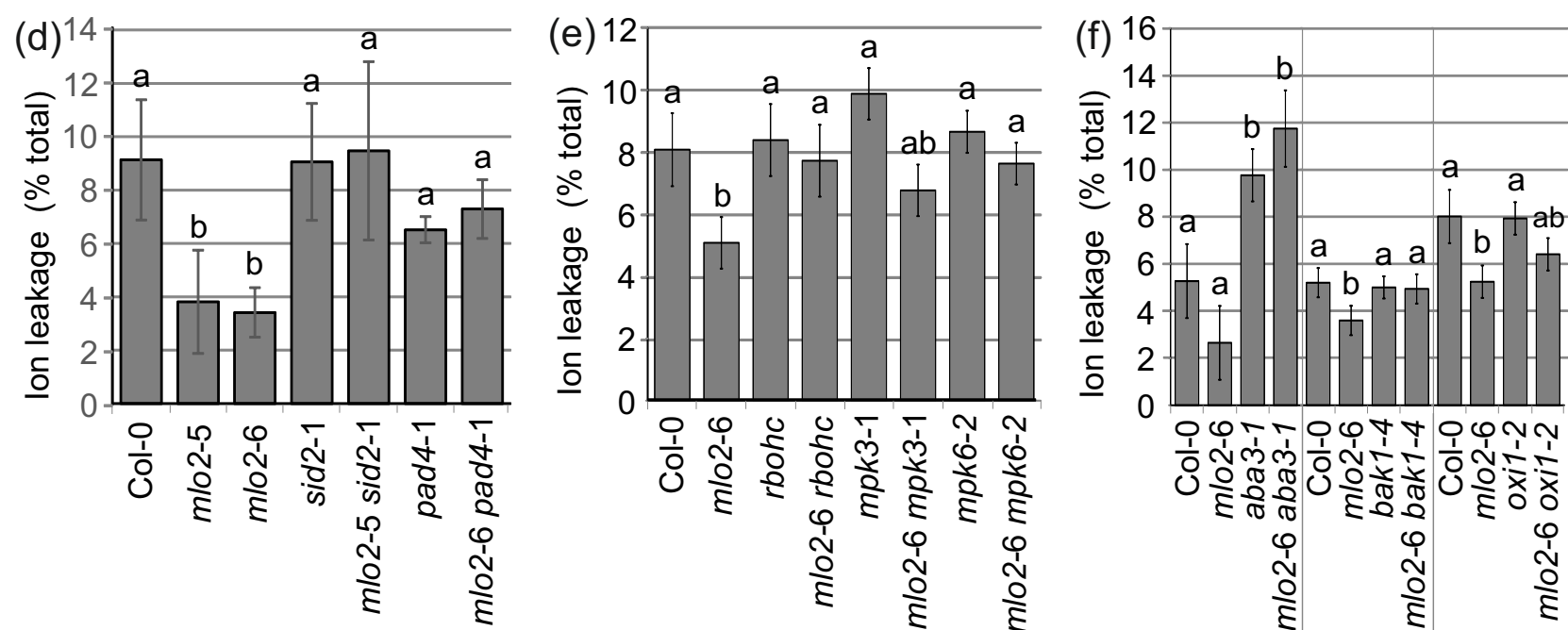
(a)

5 weeks

6 weeks

7 weeks

8 weeks

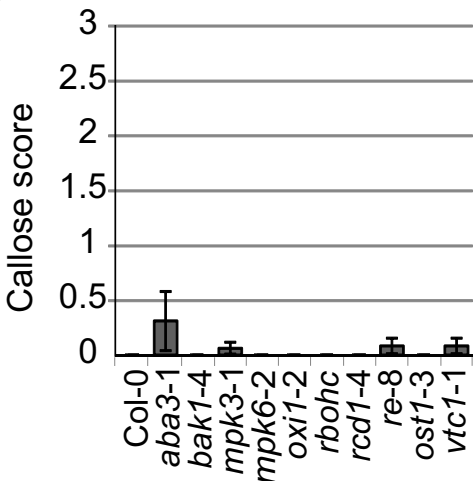

5 weeks

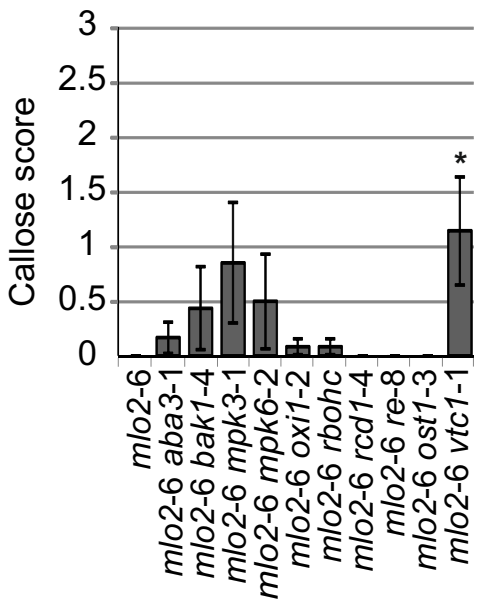

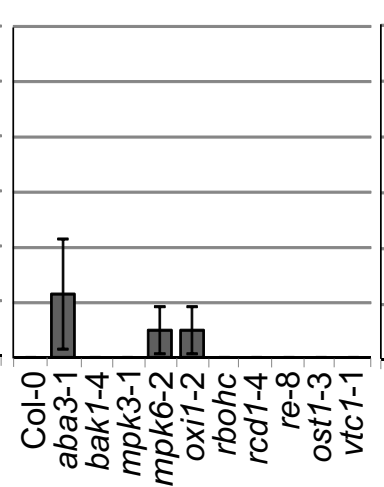

6 weeks

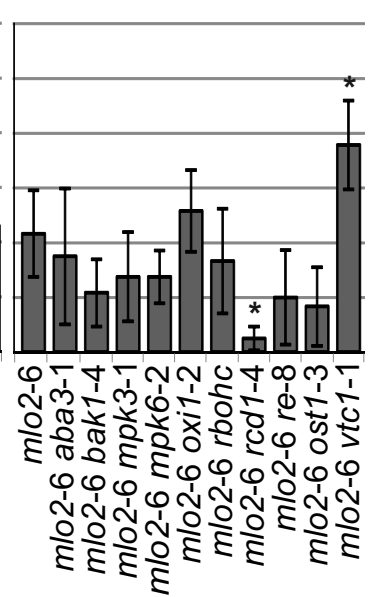

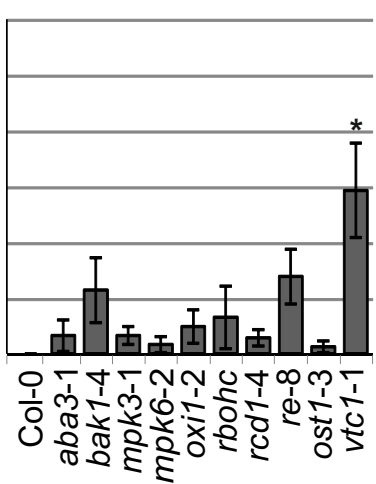

7 weeks

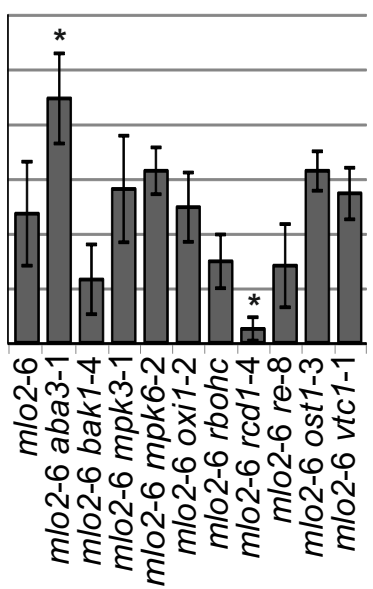

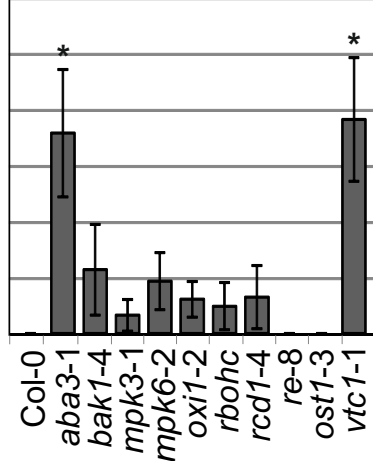

8 weeks

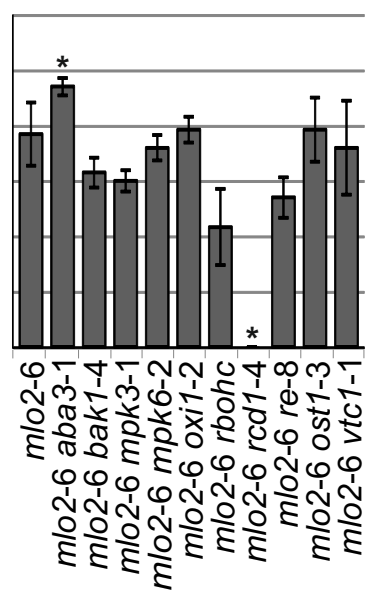

(b)
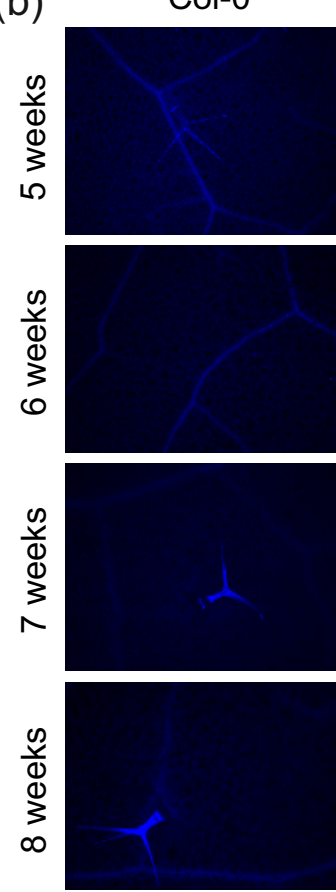

mlo2-6
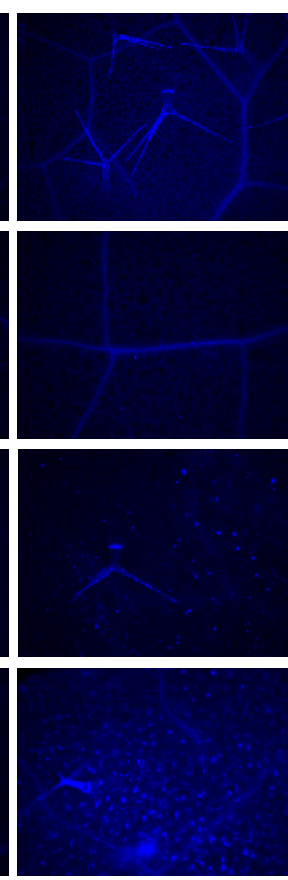

mlo2-6 aba3-1
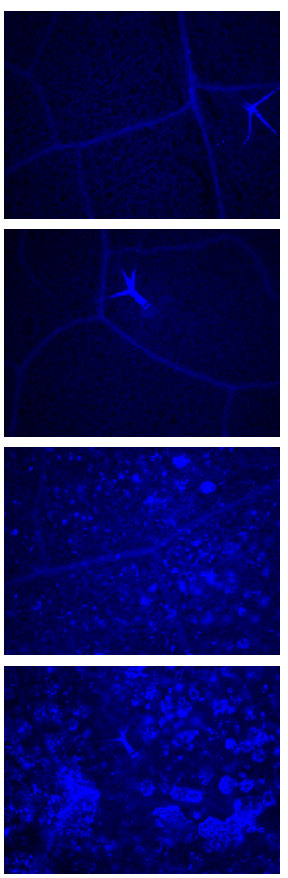

mlo2-6 rcd1-4
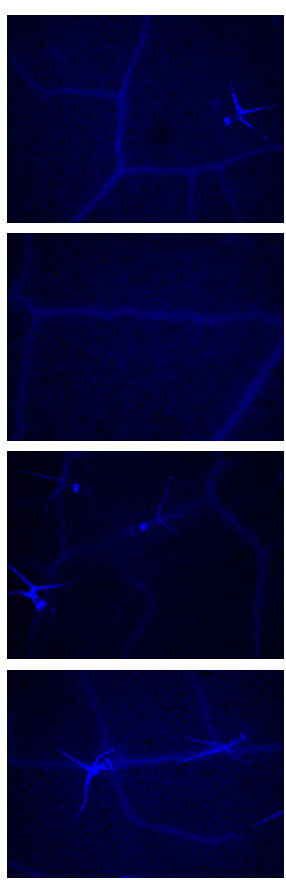

vtc1-1

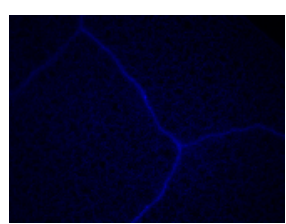

mlo2-6 vtc1-1

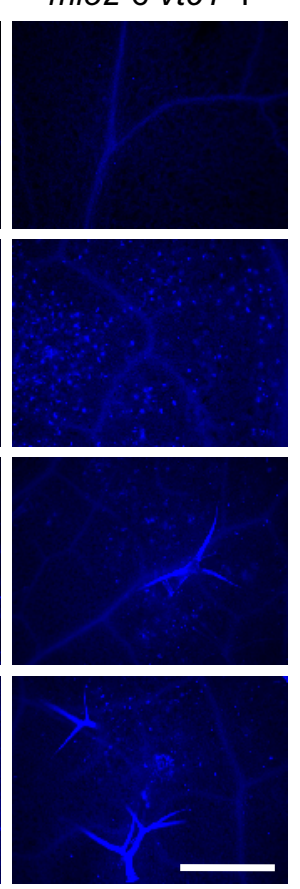


प⿻

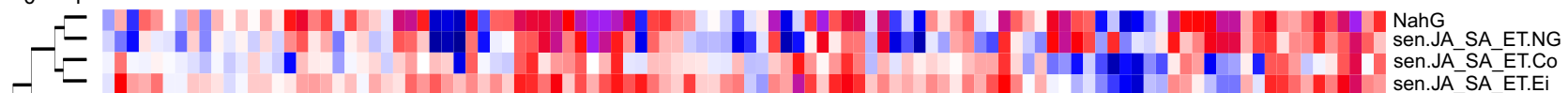

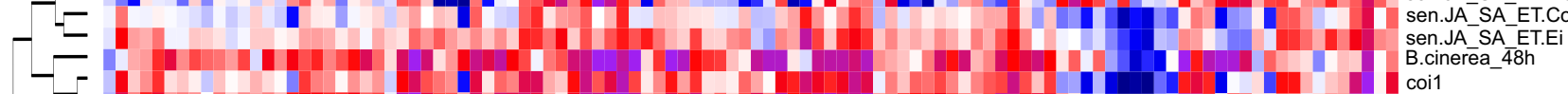

$-1-5$
-

-E

-

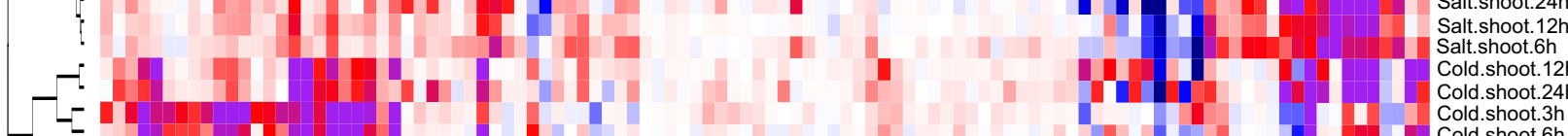

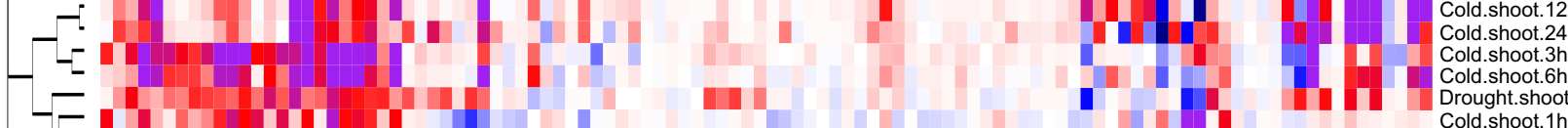

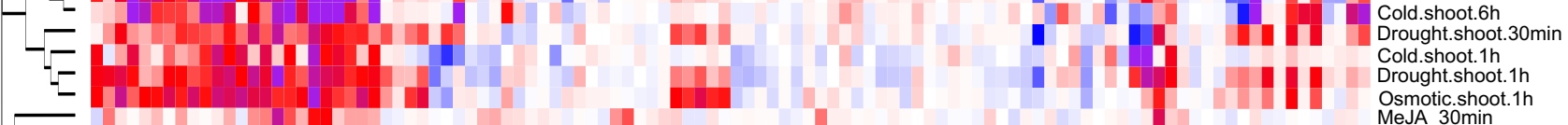

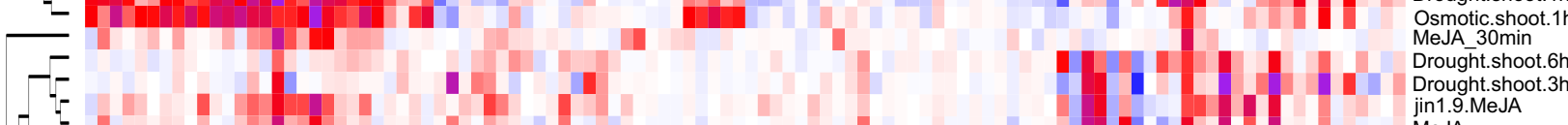

-

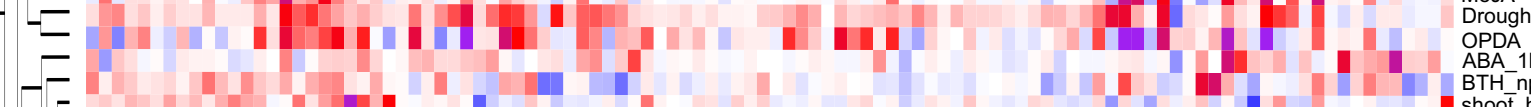

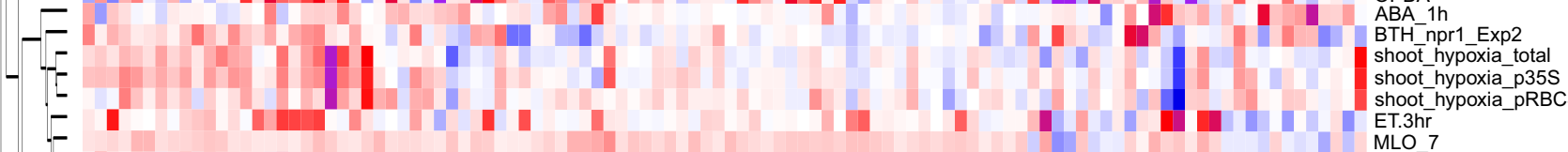

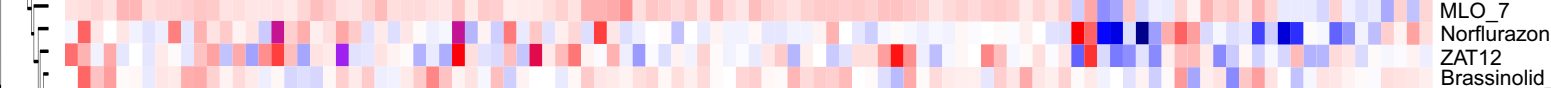

:

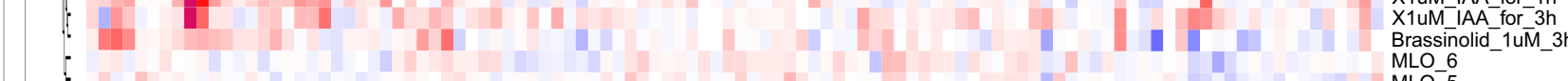

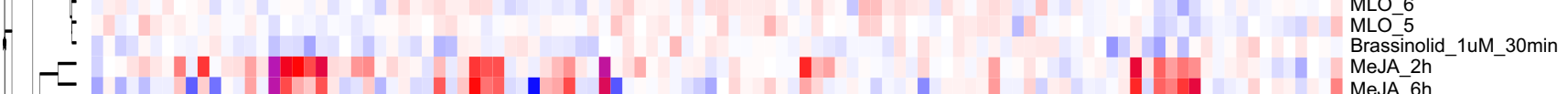

-

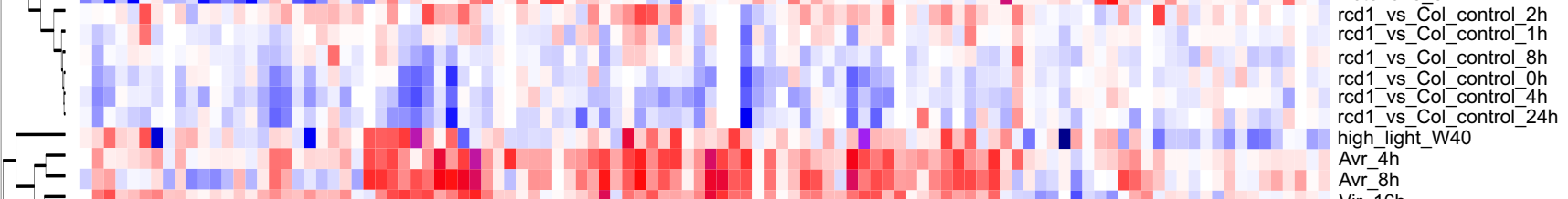

-

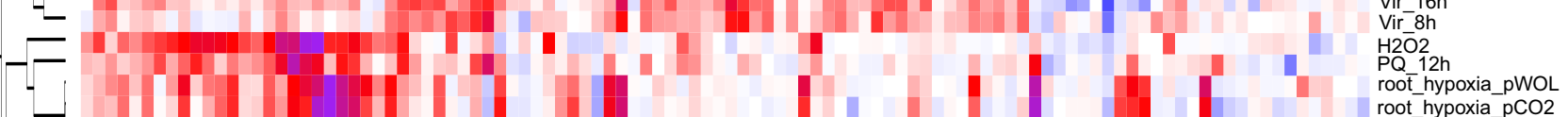

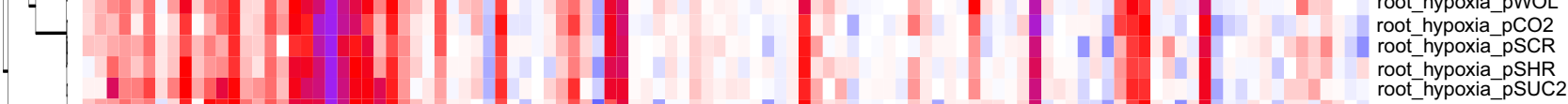

$\begin{array}{lll} & \\ 1 & & \end{array}$

$-E$
$-E$

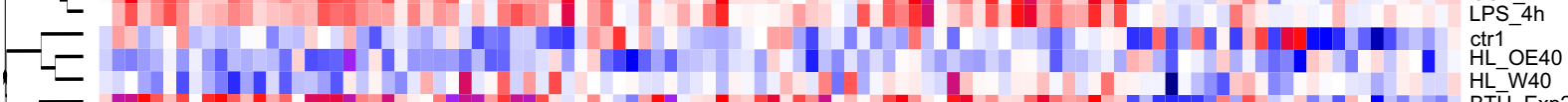

Z二
$-Z$

- ב-

-E

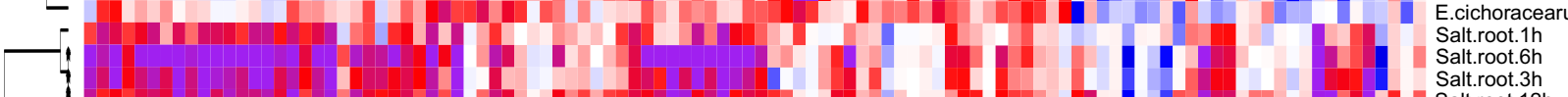

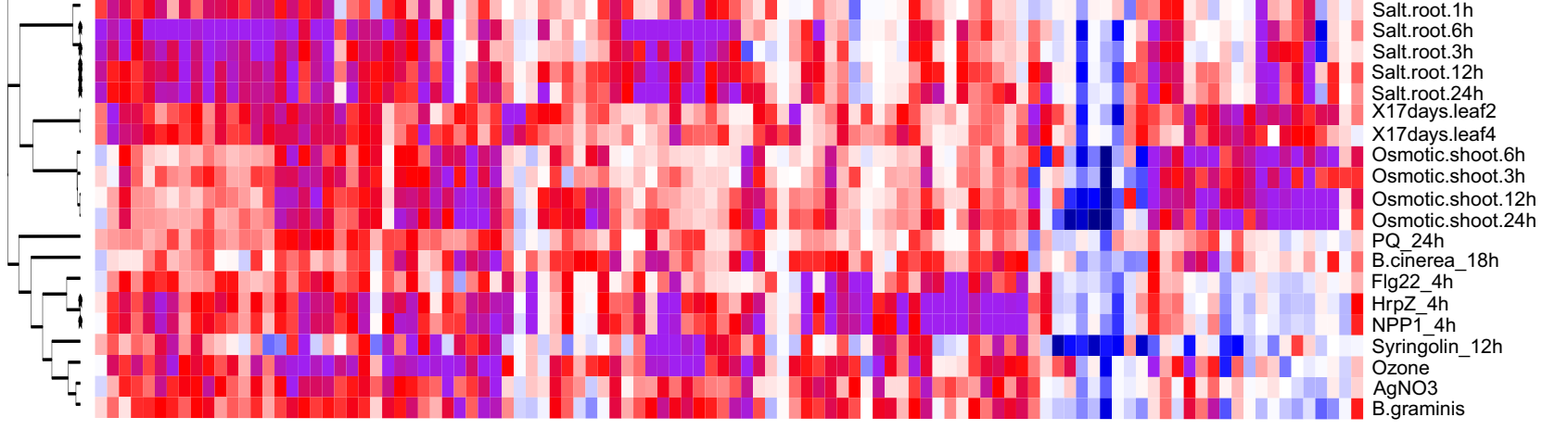

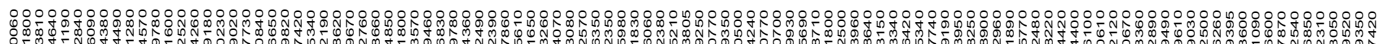

D.
D. 


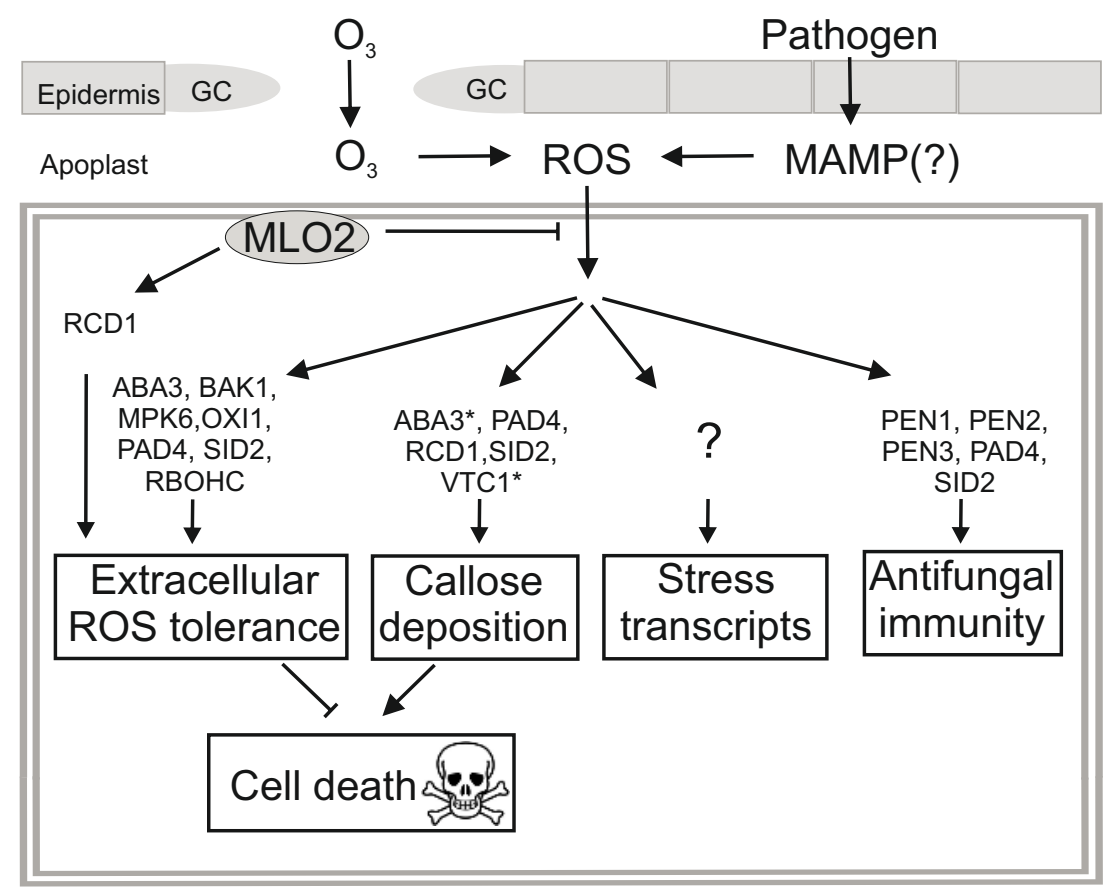


Figure S1
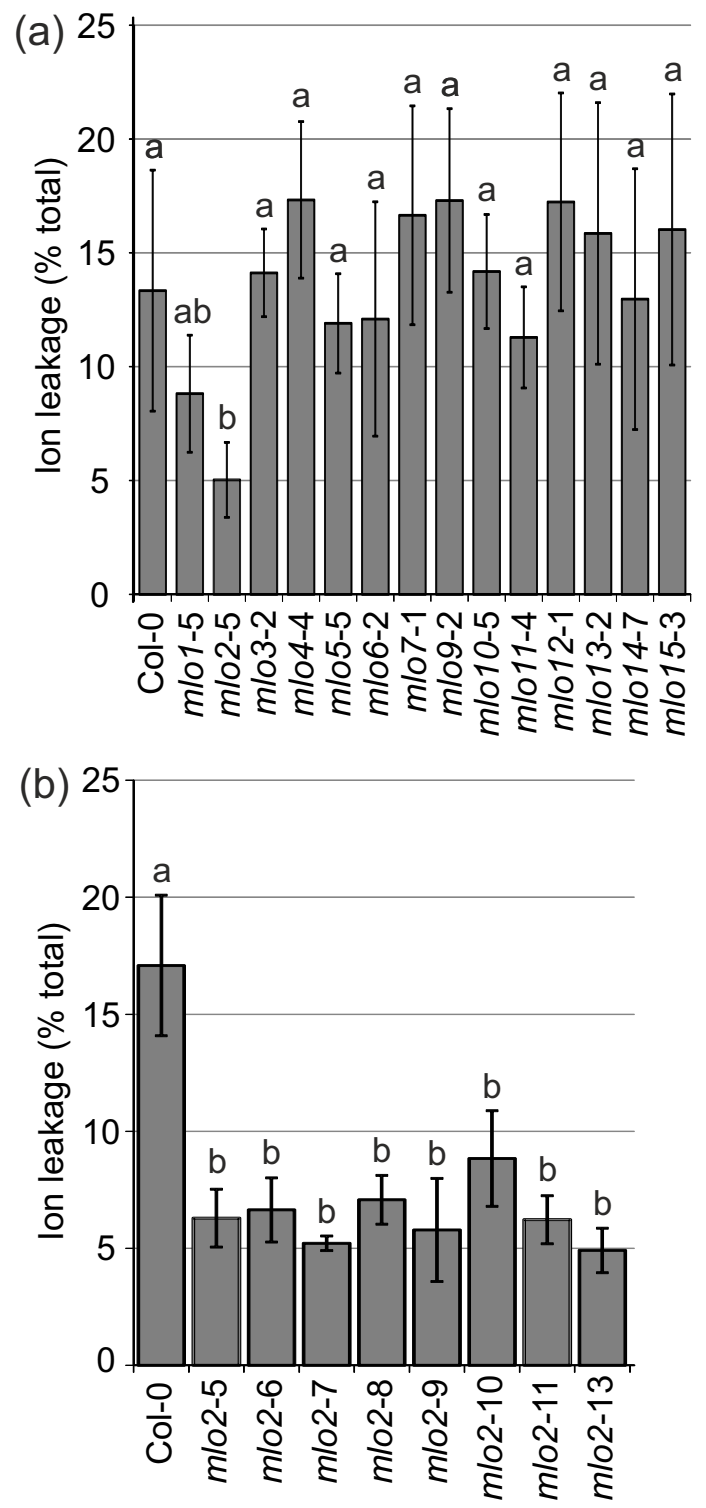

Figure S1: Enhanced $\mathrm{O}_{3}$ tolerance is specific for mlo2 mutants. (a) $\mathrm{O}_{3}$-induced electrolyte leakage from mutants for 14 of the 15 Mlo gene family members in Arabidopsis, including mlo1-5, mlo2-5, mlo3-2, mlo4-4, mlo5-2, mlo6-2, mlo7-1, mlo9-2, mlo10-5, mlo11-4, mlo12-1, mlo13-2, mlo14-7, and mlo15-3. Plants were exposed to $\mathrm{O}_{3}\left(350-400 \mathrm{nl} \mathrm{I}^{-1}\right)$ for $6 \mathrm{~h}$ and cell death assayed as electrolyte leakage. Experiments were repeated in three independent biological replicates, with data of a representative experiment shown. All data presented are means $\pm S D(n=5)$ for electrolyte leakage expressed as \% of total ions determined after disrupting leaves by boiling. Letters indicate statistically different groups based on two-way ANOVA with post-hoc analysis. (b) $\mathrm{O}_{3}$ exposure phenotypes of eight mlo2 mutant alleles in comparison to Col-0 wild-type. Lines mlo2-5, mlo2-6 and mlo2-7 comprise T-DNA insertion mutants, while mlo2-8 (G66R), mlo2-9 (S220F), mlo2-10 (D253N), mlo2-11 (D287N), and mlo2-13 (P431S) are missense point mutations. $\mathrm{O}_{3}$ exposure and data presentation are the same as in (a). 
Figure S2

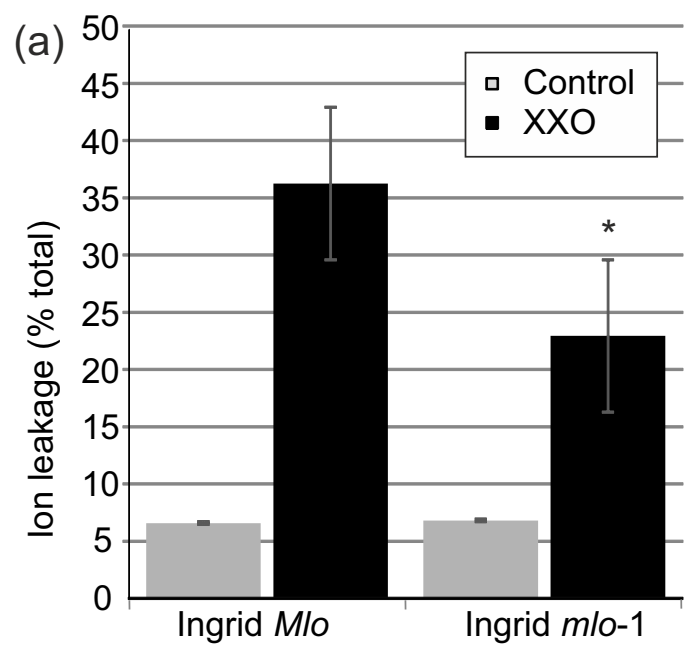

(b)

$$
\text { Ingrid Mlo }
$$

Ingrid $m / o-1$

Figure S2: Enhanced tolerance of a barley mlo mutant to extracellular ROS. (a) Electrolyte leakage from primary leaves of barley (Hordeum vulgare) wild-type (Mlo) and a near-isogenic mlo mutant (mlo-1) in cultivar "Ingrid", treated in vitro with an enzymatic superoxide generating system comprised of $1 \mathrm{mM}$ xanthine and 0.1 unit $\mathrm{ml}^{-1}$ xanthine oxidase (XXO). Data are presented as means $\pm S D(n=5)$ for ion leakage expressed as $\%$ of total ions determined after disrupting leaves by boiling. The asterisk indicates a statistically significant difference between XXO treated wild-type Ingrid Mlo and XXO treated mutant Ingrid mlo-1 based on a student t-test. (b) Photos of the basal section from leaf number two of two-week-old wild-type Mlo or mutant mlo-1 cultivar "Ingrid" barley exposed for two days to $350 \mathrm{nl} \mathrm{I}^{-1} \mathrm{O}_{3}$ and photographed $72 \mathrm{~h}$ after the beginning of the first exposure. Photos of each genotype were cropped from a single larger photo and are separated by a black line. The black scale bar in the bottom right $(=2 \mathrm{~cm})$ is valid for both photos. The experiments were repeated in three independent biological replicates, with data or photos of a single representative experiment shown. 
Figure S3
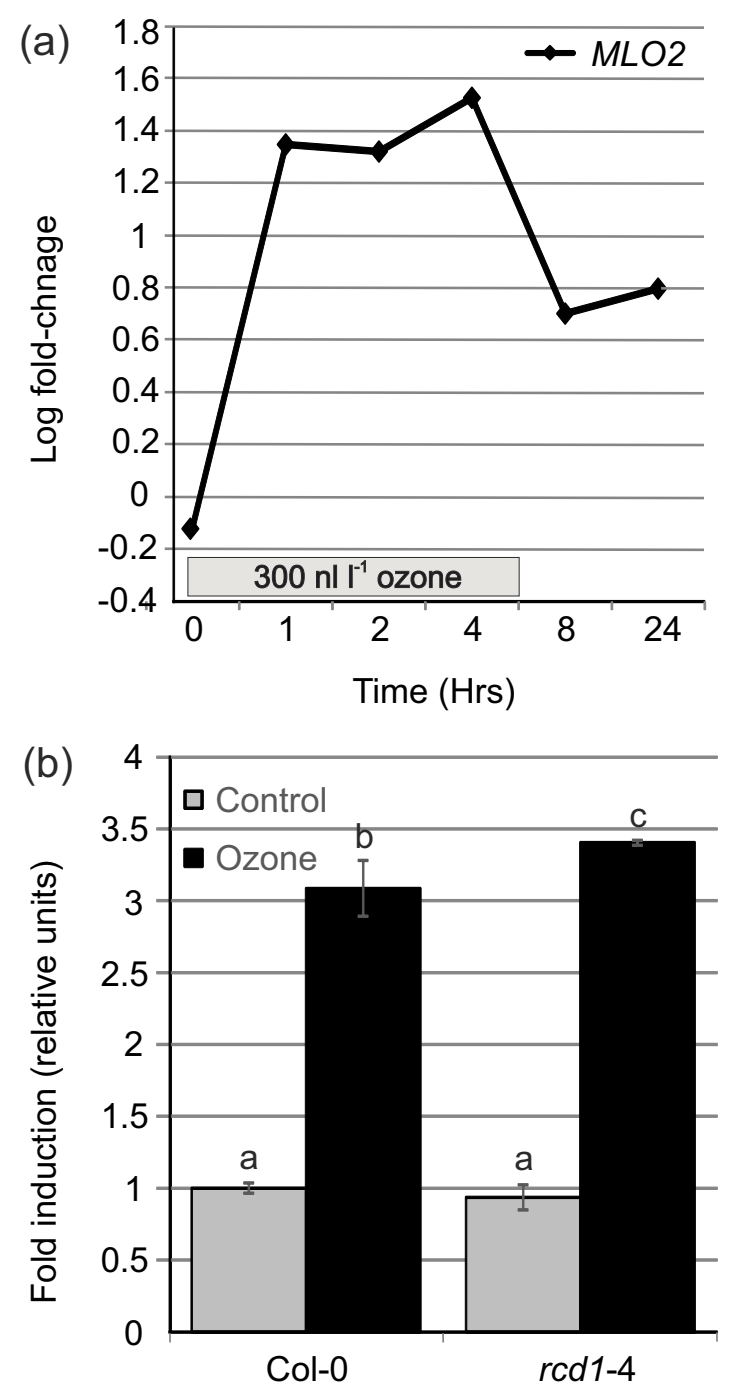

Figure S3: MLO2 gene expression. (a) Expression of $M L O 2$ mined from a publicly available $\mathrm{O}_{3}$ transcriptomics dataset (Blomster et al. 2011). The figure depicts the time course of MLO2 expression in Col-0 plants, presented as $\log _{2}$-fold change in comparison to a common control, over the course of a $6 \mathrm{~h}$ exposure to $300 \mathrm{nl}^{-1} \mathrm{O}_{3}$. (b) Quantification of $M L O 2$ transcript levels by qRT-PCR from four-weekold Col-0 wild-type and rcd1-4 mutant plants, exposed to either $1 \mathrm{~h}$ of $250 \mathrm{nl} \mathrm{I}^{-1} \mathrm{O}_{3}$ (ozone) or to "clean air" (control). Results are presented in relative units as means \pm SD of two biological replicates and were pooled and analysed. Significance groups $(P<0.05)$ are indicated by letters based on two-way ANOVA with post-hoc analysis. See Table S1 for a list of reference genes and primers used. 

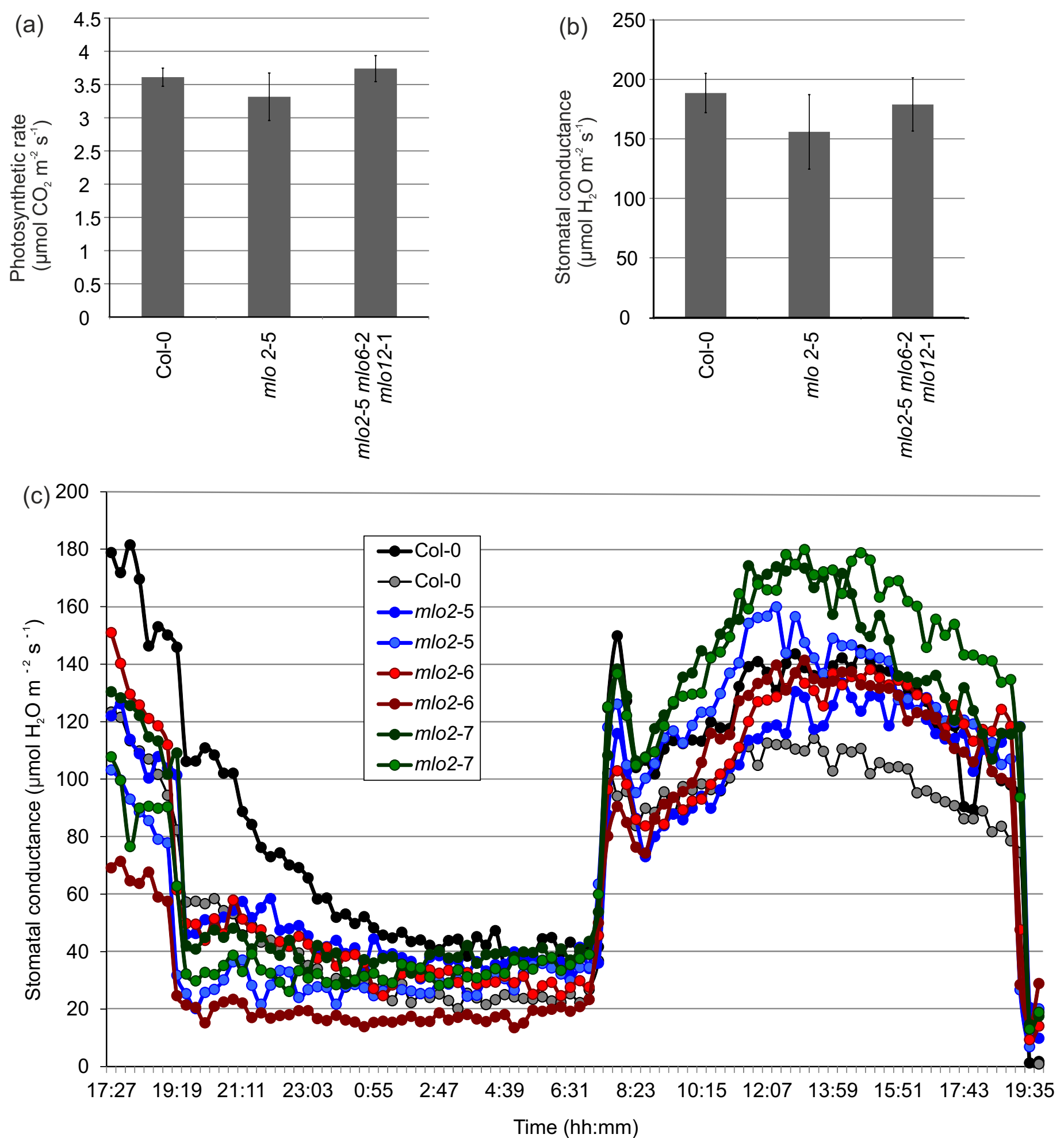

Figure S4: Stomatal and photosynthesis measurements. (a) Photosynthetic rate, indicated as $\mu \mathrm{mol} \mathrm{CO}_{2} \mathrm{~m}^{-2}$ $\mathrm{s}^{-1}$, of Col-0, mlo2-6, mlo2-5 mlo6-2 mlo12-1. Statistical analysis revealed no difference between genotypes. (b) Stomatal conductance $\left[\mathrm{G}_{\mathrm{s}}\right.$ ], indicated as $\mu \mathrm{mol} \mathrm{H}_{2} \mathrm{O} \mathrm{m} \mathrm{m}^{-2} \mathrm{~s}^{-1}$, of Col-0, mlo2-5, mlo2-5 mlo6-2 mlo12-1. Statistical analysis revealed no difference between genotypes. (c) Diurnal time course of stomatal conductance of Col-0 and m/o2 mutant plants. Stomatal conductance, presented in relative units, was recorded for two consecutive days and circadian stomatal conductance patterns for the second day are shown. Two data points each for three alleles of $\mathrm{mlo} 2$ ( $\mathrm{mlo} 2-5$, m/o2-6, and $\mathrm{mlo2}-7$ ) are displayed. 

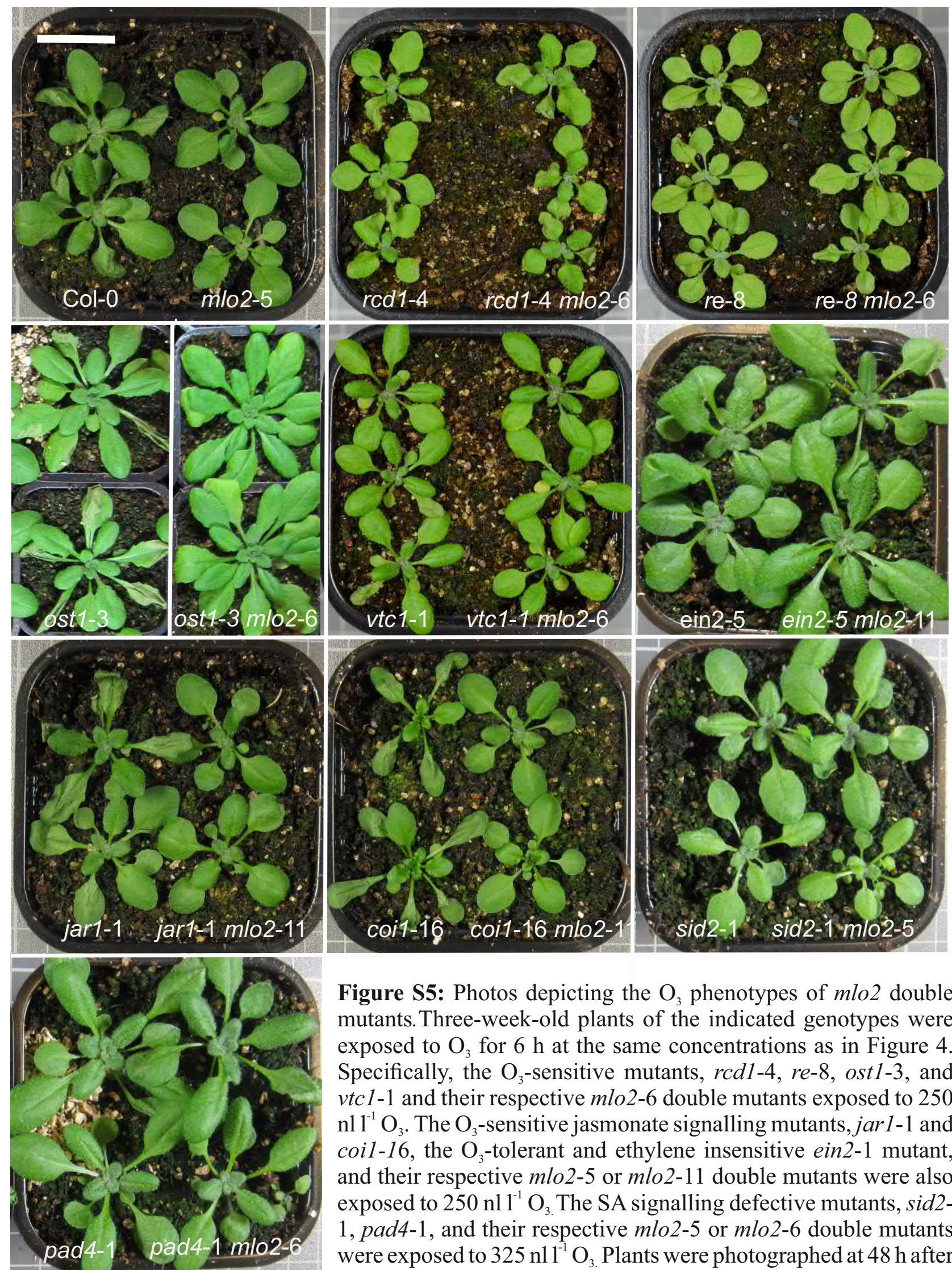

Figure S5: Photos depicting the $\mathrm{O}_{3}$ phenotypes of mlo2 double mutants. Three-week-old plants of the indicated genotypes were exposed to $\mathrm{O}_{3}$ for $6 \mathrm{~h}$ at the same concentrations as in Figure 4. Specifically, the $\mathrm{O}_{3}$-sensitive mutants, $r c d 1-4, r e-8$, ost $1-3$, and $v t c 1-1$ and their respective mlo2-6 double mutants exposed to 250 $\mathrm{nl} \mathrm{l}^{-1} \mathrm{O}_{3}$. The $\mathrm{O}_{3}$-sensitive jasmonate signalling mutants, jar $1-1$ and coil-16, the $\mathrm{O}_{3}$-tolerant and ethylene insensitive ein2-1 mutant, and their respective mlo2-5 or mlo2-11 double mutants were also exposed to $250 \mathrm{nl} \mathrm{l}^{-1} \mathrm{O}_{3}$. The SA signalling defective mutants, sid21, pad4-1, and their respective mlo2-5 or mlo2-6 double mutants were exposed to $325 \mathrm{nl} \mathrm{l}^{-1} \mathrm{O}_{3}$. Plants were photographed at $48 \mathrm{~h}$ after the beginning of the exposure. All experiments were repeated in at least two independent biological replicates, with data from a representative experiment shown. Separate photos that have been composited are separated by white lines. The white scale bar $(=2$ $\mathrm{cm}$ ) in the top left is valid for all images. 
Figure S6
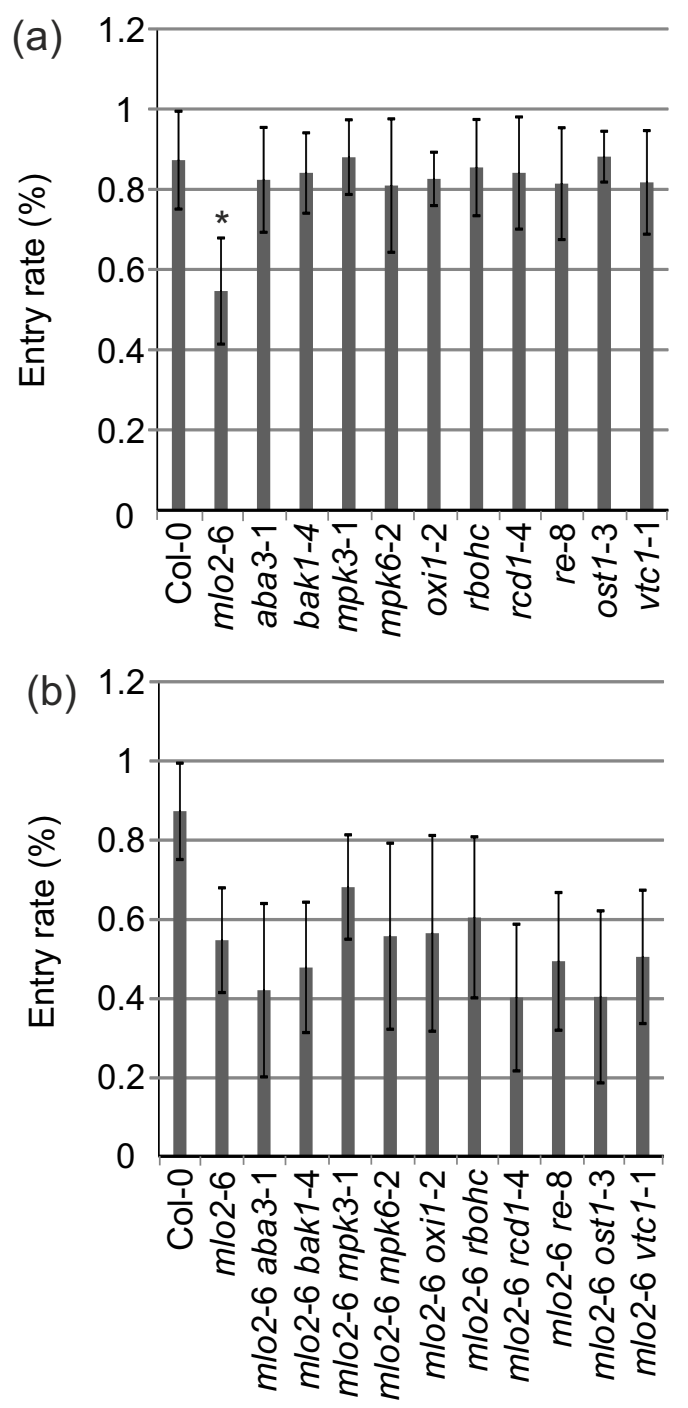

Figure S6: The mlo2 double mutants exhibit unaltered powdery mildew entry rates. Six-week-old plants of the indicated genotypes were challenged with $G$. orontii. The proportion of cells of the indicated genotypes successfully entered by the fungal pathogen at $48 \mathrm{~h}$ after inoculation are shown. Results are presented as the means \pm SD of pooled data from five independent biological replicates. At least 100 interaction sites were manually scored by microscopic examination of three to five leaves per biological replicate. The absence of an asterisk indicates no significant difference of single mutants (apart from mlo2-6) to Col-0 (a) or mlo2-6 double mutants compared to the mlo2-6 single mutant (b). Note that the same Col-0 and mlo2-6 controls are plotted in both graphs for reference. Significance of pooled data was calculated using post-hoc analysis by computing contrasts from linear models and subjecting the $\mathrm{P}$ values to single-step error correction. 

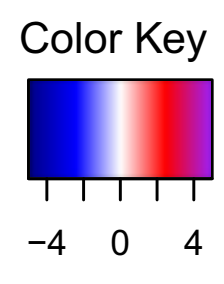

Value
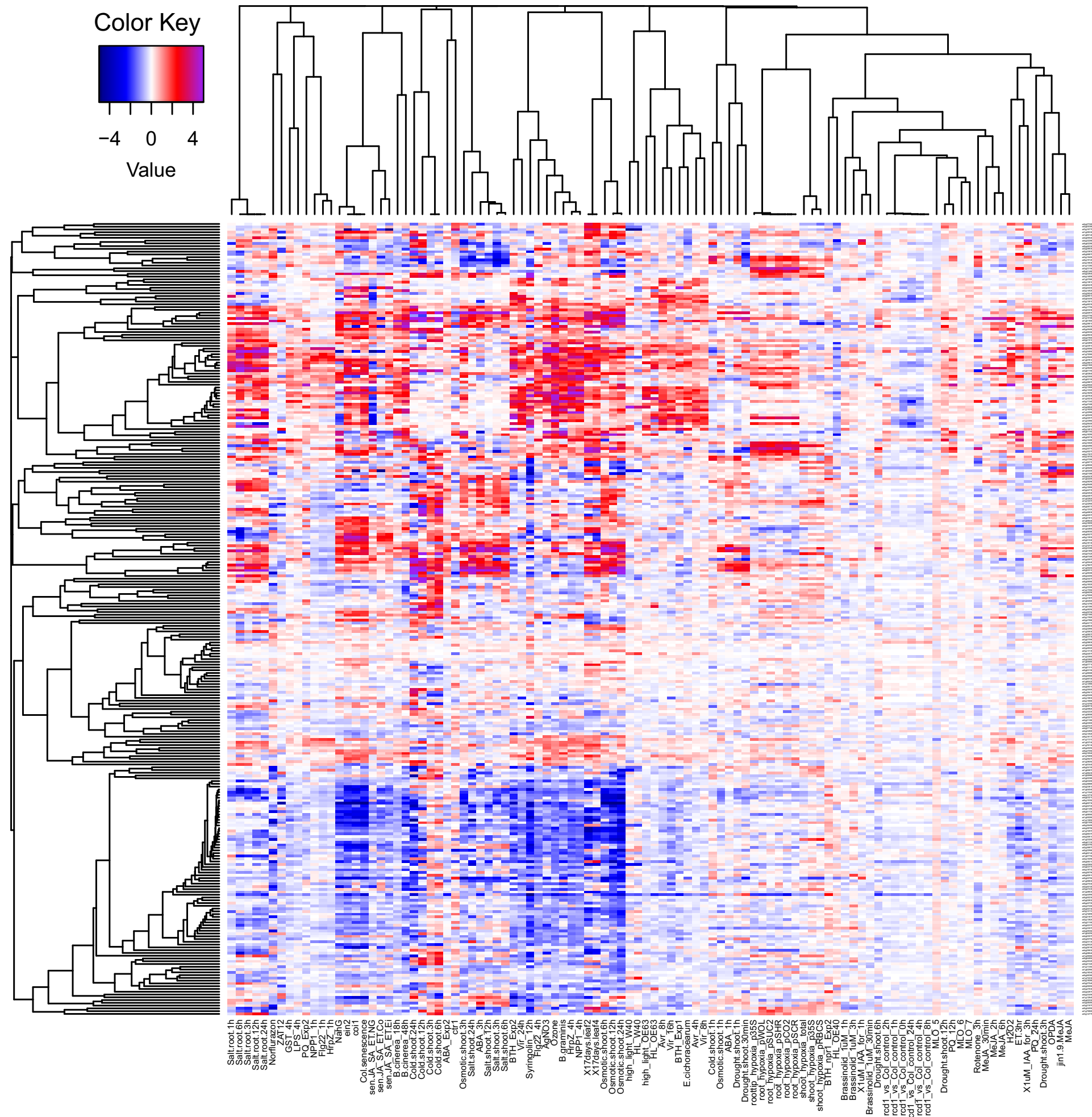

Figure S7: Clustering analysis of MLO-regulated genes: week five time point genes. Hierarchical clustering analysis of expression profiles for MLO-regulated genes from the five-week-old time point. We used all 297 MLO2-, MLO6-, MLO12-regulated genes from the week five gene set(Consonni et al. 2010; see Table S2a for gene list) and subjected their expression profiles to hierarchical clustering analysis. A collection of publicly available transcript profiling experiments related to ROS, abiotic stress, biotic stress, hormone signalling, and transcriptional regulators was used (see Table S2i for list of experiment accession numbers). 
Figure S8
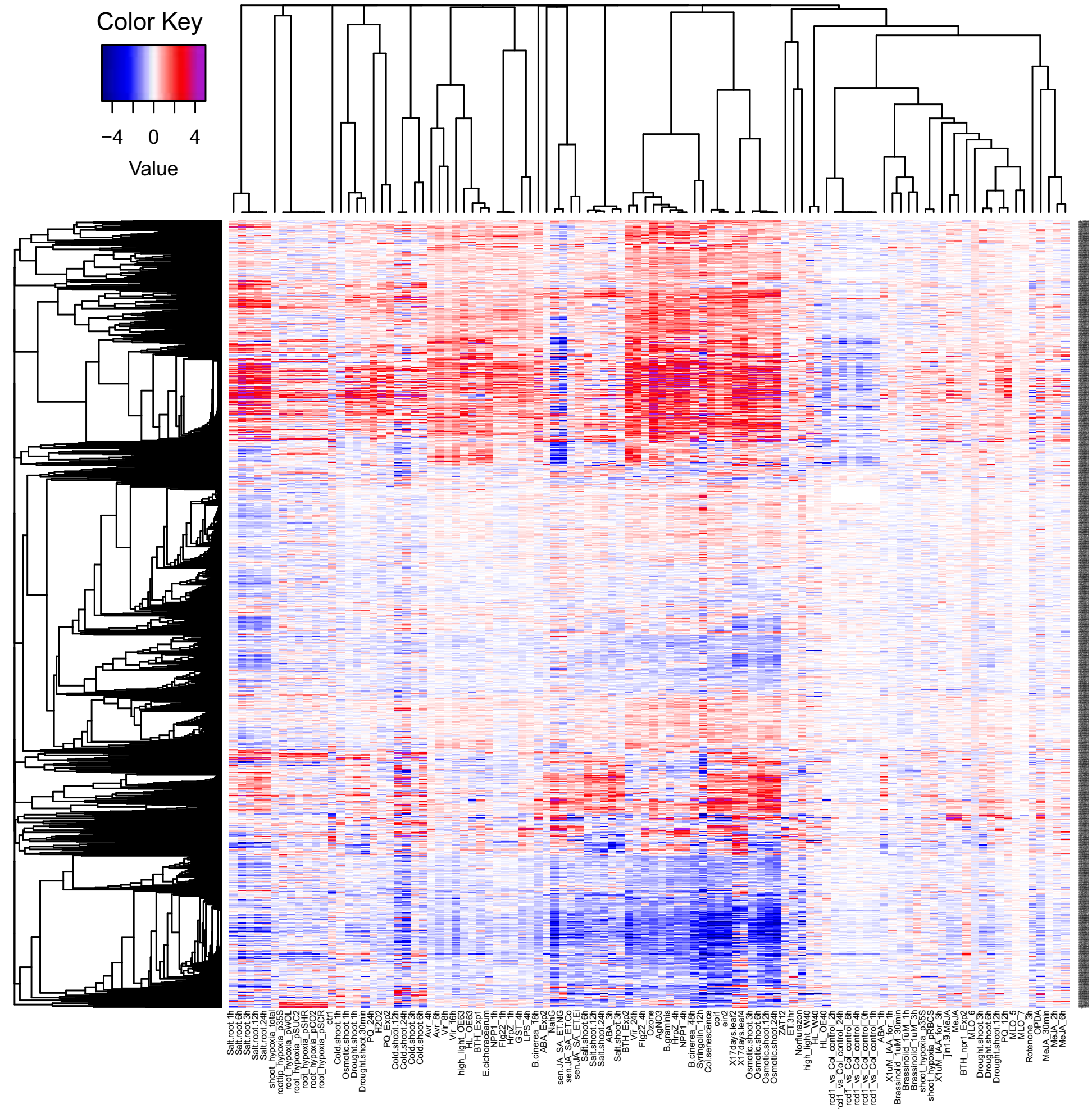

Figure S8: Clustering analysis of MLO regulated genes: week six time point genes. Hierarchical clustering analysis of expression profiles for MLO-regulated genes from the six-week-old time point. We used all 1134 MLO2-, MLO6-, MLO12-regulated genes from the week six gene set(Consonni et al. 2010; see Table S2a for gene list) and subjected their expression profiles to hierarchical clustering analysis. A collection of publicly available transcript profiling experiments related to ROS, abiotic stress, biotic stress, hormone signalling, and transcriptional regulators was used (see Table S2i for list of experiment accession numbers). 

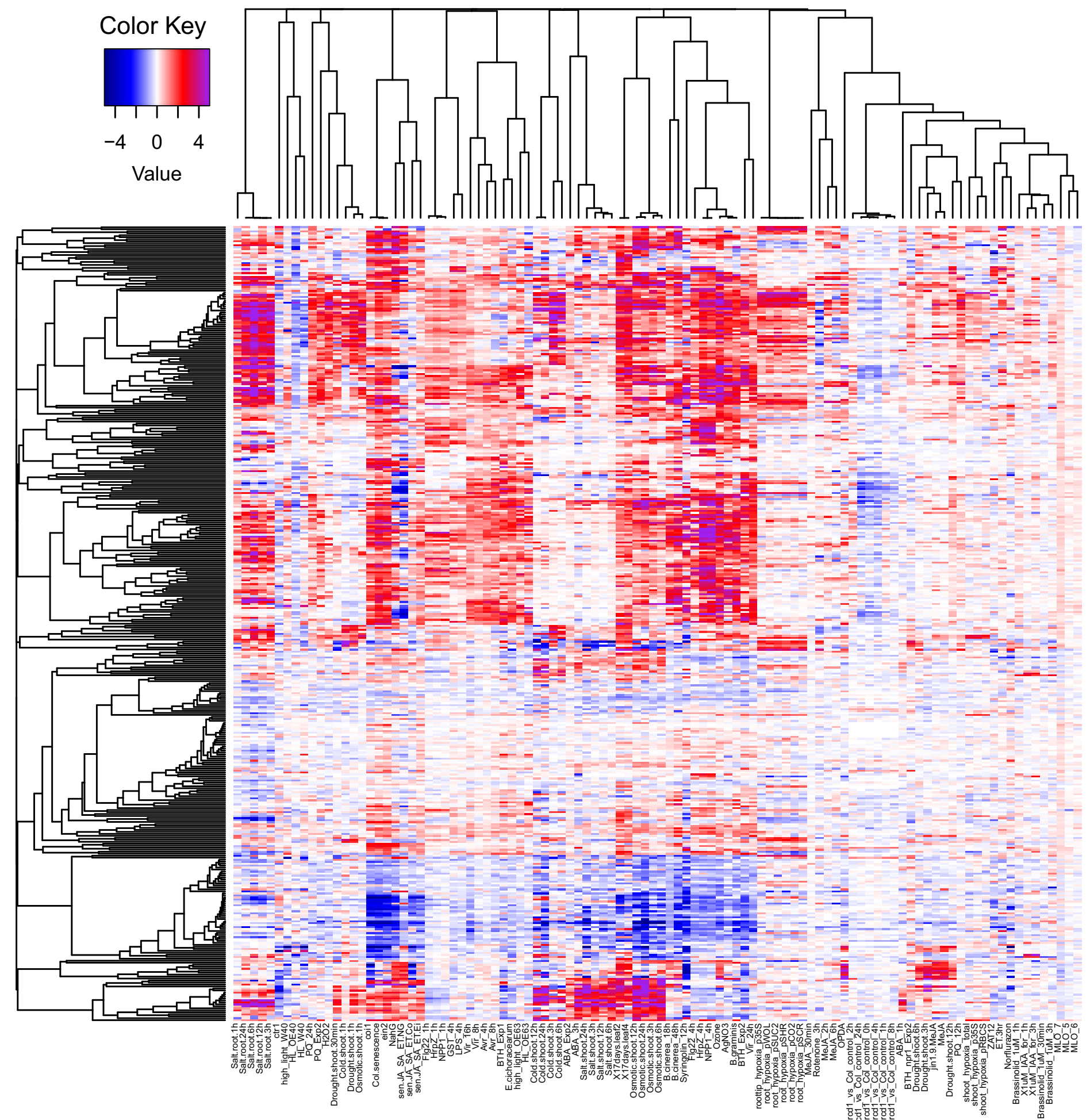

Figure S9: Clustering analysis of MLO regulated genes: week seven time point genes. Hierarchical clustering analysis of expression profiles for MLO-regulated genes from the seven-week-old time point. We used all 413 MLO2-, MLO6-, MLO12-regulated genes from the week seven gene set (Consonni et al. 2010; see Table S2a for gene list) and subjected their expression profiles to hierarchical clustering analysis. A collection of publicly available transcript profiling experiments related to ROS, abiotic stress, biotic stress, hormone signalling, and transcriptional regulators was used (see Table S2i for list of experiment accession numbers). 
Supplemental Table S1: Primers used in this study

Genotyping primers

\begin{tabular}{llll}
\hline Name & Full name & Allelic to & AGI code \\
\hline rbohC & respiratory burst oxidase homolog c & rhd2-4 & At5g51060 \\
rcd1-4 & radical-induced cell death1 & At1g32230 \\
oxi1-2 & oxidative signal-inducible1 & At3g25250 \\
ost1-3 & open stomata 1-3 & srk2e & At4g33950 \\
mpk3-1 & mitogen-activated protein kinase 3-1 & & At3g45640 \\
mpk6-2 & mitogen-activated protein kinase 6-2 & & At2g43790 \\
vtc1-1 & vitamin c deficient 1 & & At2g39770 \\
aba3-1 & aba deficient3-1 & & At1g16540 \\
bak1-4 & bri1-associated receptor kinase & & At4g33430 \\
re-8 & reticulata-8 & At2g37860
\end{tabular}

References

Chinchilla D, Zipfel C, Robatzek S, Kemmerling B, Nürnberger T, Jones J[ Conklin PL, Norris SR, Wheeler GL, Williams EH, Smirnoff N, Last RL. 199 Foreman J, Demidchik V, Bothwell JHF, Mylona P, Miedema H, Torres MA: Jaspers P, Blomster T, Brosché M, Salojärvi J, Ahlfors R, Vainonen JP, Re Nakagami H, Soukupová H, Schikora A, Zárský V, Hirt H. 2006. A MitogenOvermyer K, Kollist H, Tuominen H, Betz C, Langebartels C, Wingsle G, $\mathrm{K}_{\mathbf{i}}$ Rentel MC, Lecourieux D, Ouaked F, Usher SL, Petersen L, Okamoto H, Kı Wang H, Ngwenyama N, Liu Y, Walker JC, Zhang S. 2007. Stomatal Develo| Yoshida R, Hobo T, Ichimura K, Mizoguchi T, Takahashi F, Aronso J, Ecke 


\begin{tabular}{lll}
\hline Mutant type & Phenotypes & genotyping \\
\hline Transposon insertion & & PCR \\
GABI_229D11 & $\begin{array}{l}\text { O3 sensitive; smaller rosette, short } \\
\text { petioles, distorted leaves with serated margins }\end{array}$ & PCR \\
GABI_355H08 & & PCR \\
SALK_008068 & & PCR \\
SALK_151594 & & PCR \\
SALK_073907 & & PCR \\
EMS & $\begin{array}{l}\text { O3 sensitive; short root and much more root } \\
\text { hairs with seedings. smaller rosette and }\end{array}$ & CAPS/Styl \\
EMS & narrower leaves with adult plants & \\
SALK_116202 & small rosette size and permeable cuticle & CAPS/Mnll \\
EMS & & PCR
\end{tabular}

JG, Felix G, Boller T. 2007. A flagellin-induced complex of the receptor FLS2 and BAK1 19. Genetic evidence for the role of GDP-mannose in plant ascorbic acid (vitamin C) biosy , Linstead P, Costa S, Brownlee C, Jones JDG, et al. 2003. Reactive oxygen species \& əddy RA, Immink R, Angenent G, Turck F. 2009. Unequally redundant RCD1 and SRO activated Protein Kinase Kinase Kinase Mediates Reactive Oxygen Species Homeo angasjärvi S, Brader G, Mullineaux P, Kangasjärvi J. 2008. Complex phenotypic profil night H, Peck SC, Grierson CS, Hirt H, et al. 2004. OXI1 kinase is necessary for oxidat oment and Patterning Are Regulated by Environmentally Responsive Mitogen-Activated $F$ ir JR, Shinozaki K. 2002. ABA-Activated SnRK2 Protein Kinase is Required for Dehydra 
Forward primer

TAGCTTCTCCATGTGACCGCG

AGGTGTGAAGATGGATTCGG

CCTTTCCAAACAAAGCAAGTG

TTTGCCGATTATGCACGATA

CGAGCAATCTTCTGTTGAACG

GCCTCAGATGCCTGGGATTGAGAATATTC

TGCATTTTCAGGAAAAGGAGTT

TCATTCTTTCTACTGCTCCTGATTT

CTATCTCTCCTACACCGCCATC

TGTGGCATTGTAGGTCAAGG

initiates plant defence. Nature 448: 497-500.

nthesis. Proceedings of the National Academy of sroduced by NADPH oxidase regulate plant cell gl 1 mediate stress and developmental responses ar stasis in Arabidopsis. Journal of Biological $\mathrm{Cr}$ es leading to ozone sensitivity in Arabidopsis thali ive burst-mediated signalling in Arabidopsis. Natu 'rotein Kinases in Arabidopsis. The Plant Cell Onı tion Stress Signaling in Arabidopsis. Plant and $C \epsilon$ 


\begin{tabular}{ll}
\hline Reverse primer & Source or reference \\
\hline ATCTAAAGCTAGATGCCTTAGC & Foreman et al., 2003 \\
GGGGTTTTTGCTCCTGTTTC & Jaspers et al., 2009 \\
AAGAAACGTCTCTTCCGCTTC & Rentel et al., 2004 \\
AGGAGCGATGTAAGCAGGAG & Yoshida et al., 2002 \\
GCTGCTGCACTTCTAACCGTATG & Nakagami et al., 2006 \\
AGAGTGGCTTACGGTCCATT & Nakagami et al., 2006
\end{tabular}

TTAGCAAAATCAACAAGGGGCCTTG Conklin et al., 1999

GGTGAAGCAAATGAACTTATGATG Xiong et al., 2001

CTTGCAGATCCCAACGCAATACG Chinchilla et al., 2007

CACCTGCAATTAATCAACCA Overmyer et al., 2008

Sciences 96: 4198-4203.

rowth. Nature 422: 442-446.

רd interact with transcription factors. The Plant Journal 60: 268-279.

iemistry 281: 38697-38704.

ana mutants. Plant, Cell \& Environment 31: 1237-1249.

Ire 427: 858-861.

line 19: 63-73.

!ll Physiology 43: 1473-1483. 


\title{
Supplemental Table S2i: Genes and Experiments used in Figure 6.
}

\author{
Click here to jump to Experiments below.
}

${ }^{\ddagger}$ Gene descriptions sourced from from TAIR (https://www.arabidopsis.org/tools/

${ }^{\dagger}$ Data sources are from: The National Center for Bioinformatics Gene expressic The Nottingham Arabidopsis Stock Centre Arays (NASC Arrays); and the Harve

\section{Genes $^{\ddagger}$}

\section{AGI codes}

AT4G24380

AT2G20670

AT1G66100

AT4G12500

AT3G04640

AT4G29780

AT1G68620

AT3G28220

AT2G32190

AT3G54400

AT3G48640

AT1G66090

AT3G01830

AT5G39520

AT1G56060

AT1G16850

AT5G52760

AT5G38900

AT1G19020

AT2G37870

AT4G16260

AT4G39670

AT1G35210

AT3G02840

AT3G13950

AT2G44240

AT1G51890

AT5G52390

AT3G18250

AT1G26420

AT1G30700

AT1G51090

AT4G12490

AT5G38710

AT1G32960

AT4G23810

AT2G43570

AT5G45340
Gene Model Name Gene Model Description Gene Model TJ

AT4G24380.1 dihydrofolate reductase;(source:Araport11)

AT2G20670.1 sugar phosphate exchanger, putative (DUF5C

AT1G66100.1 Predicted to encode a PR (pathogenesis-rela

AT4G12500.1 Bifunctional inhibitor/lipid-transfer protein/see

AT3G04640.1 glycine-rich protein;(source:Araport11) proteir

AT4G29780.1 nuclease;(source:Araport11) protein_codin

AT1G68620.1 alpha/beta-Hydrolases superfamily protein;(si

AT3G28220.1 TRAF-like family protein;(source:Araport11)

AT2G32190.1 cysteine-rich/transmembrane domain A-like $p$

AT3G54400.1 Eukaryotic aspartyl protease family protein;(s

AT3G48640.1 transmembrane protein;(source:Araport11)

AT1G66090.1 Disease resistance protein (TIR-NBS class);(:

AT3G01830.1 Calcium-binding EF-hand family protein;(sour

AT5G39520.1 hypothetical protein (DUF1997);(source:Arap

AT1G56060.1 cysteine-rich/transmembrane domain protein

AT1G16850.1 transmembrane protein;(source:Araport11)

AT5G52760.1 Copper transport protein family;(source:Arapc

AT5G38900.1 Thioredoxin superfamily protein;(source:Arap

AT1G19020.1 CDP-diacylglycerol-glycerol-3-phosphate 3-pr

AT2G37870.1 Bifunctional inhibitor/lipid-transfer protein/see

AT4G16260.1 Encodes a putative beta-1,3-endoglucanase 1

AT4G39670.1 Glycolipid transfer protein (GLTP) family prote

AT1G35210.1 hypothetical protein;(source:Araport11) protei

AT3G02840.1 ARM repeat superfamily protein;(source:Arap

AT3G13950.1 ankyrin;(source:Araport11) protein_coding

AT2G44240.1 NEP-interacting protein (DUF239);(source:Ar:

AT1G51890.1 Leucine-rich repeat protein kinase family prot

AT5G52390.1 PAR1 protein;(source:Araport11) protein_cod

AT3G18250.1 Putative membrane lipoprotein;(source:Arapo

AT1G26420.1 FAD-binding Berberine family protein;(source

AT1G30700.1 FAD-binding Berberine family protein;(source

AT1G51090.1 Heavy metal associated domain containing p

AT4G12490.1 Encodes a member of the AZI family of lipid $t$ AT5G38710.1 Methylenetetrahydrofolate reductase family $p$ AT1G32960.1 Subtilase family protein;(source:Araport11)

AT4G23810.1 member of WRKY Transcription Factor; Grou AT2G43570.1 chitinase;(source:Araport11) protein_coding AT5G45340.1 Encodes a protein with ABA 8'-hydroxylase ar 
AT4G11280

AT5G24420

AT1G32350

AT1G33960

AT3G12500

AT5G20230

AT3G61190

AT3G48360

AT5G17860

AT5G42380

AT1G76650

AT3G50770

AT5G27420

AT4G15610

AT3G44260

AT3G22120

AT1G29395

AT2G42540

AT4G23600

AT4G23150

AT2G30770

AT4G24570

AT4G10500

AT4G12480

AT1G64360

AT5G61600

AT2G40610

AT1G19250

AT2G19190

AT1G56600

AT1G09350

AT1G61800

AT1G69930

AT2G29460

AT3G47420

AT2G18050

AT1G51800

AT1G13340

AT1G19180

AT1G73260

AT1G72520

AT5G52310

AT3G50060

AT2G39030

AT5G39610

AT1G52890

AT3G04070
AT4G11280.1

AT5G24420.1

AT1G32350.1

AT1G33960.1

AT3G12500.1

AT5G20230.1

AT3G61190.1

AT3G48360.1

AT5G17860.1

AT5G42380.1

AT1G76650.1

AT3G50770.1

AT5G27420.1

AT4G15610.1

AT3G44260.1

AT3G22120.1

AT1G29395.1

AT2G42540.2

AT4G23600.1

AT4G23150.1

AT2G30770.1

AT4G24570.1

AT4G10500.1

AT4G12480.1

AT1G64360.1

AT5G61600.1

AT2G40610.1

AT1G19250.1

AT2G19190.1

AT1G56600.1

AT1G09350.1

AT1G61800.1

AT1G69930.1

AT2G29460.1

AT3G47420.1

AT2G18050.1

AT1G51800.1

AT1G13340.1

AT1G19180.1

AT1G73260.1

AT1G72520.1

AT5G52310.1

AT3G50060.1

AT2G39030.1

AT5G39610.1

AT1G52890.1

AT3G04070.1 encodes a a member of the 1-aminocycloprop Encodes a cytosolic 6-phosphogluconolacton alternative oxidase 1D;(source:Araport11) Identified as a gene that is induced by avirule encodes a basic chitinase involved in ethylen Encodes a Al-stress-induced gene. Along witl Encodes a protein with a $\mathrm{C} 2$ domain that binc encodes a protein (BT2) that is an essential c calcium exchanger 7;(source:Araport11) prot calmodulin like 37;(source:Araport11) protei calmodulin-like 38;(source:Araport11) protei calmodulin-like 41;(source:Araport11) protei Encodes CNI1 (Carbon/Nitrogen Insensitive1 Uncharacterized protein family (UPF0497);(sc Encodes one of the homologs of the yeast $\mathrm{C}($ cell wall-plasma membrane linker protein hon Integral membrane protein in the inner envel A cold-regulated gene whose product is targe Encodes cystine lyase which is expected to $b$ Encodes a cysteine-rich receptor-like protein putative cytochrome P450 protein_codinc Encodes one of the mitochondrial dicarboxyle 2-oxoglutarate (2OG) and Fe(II)-dependent o Encodes a putative lipid transfer protein, vern $S A Q R$ is a clade specific protein present in si encodes a member of the ERF (ethylene resk member of Alpha-Expansin Gene Family. Naı FMO1 is required for full expression of TIR-NI Encodes a receptor-like protein kinase that is GolS2 is a galactinol synthase that catalyzes Predicted to encode a galactinol synthase glucose6-Phosphate/phosphate transporter 2 Encodes glutathione transferase belonging to Encodes glutathione transferase belonging to Encodes a Pi starvation-responsive protein $\mathrm{A}$ encodes a structurally divergent linker histone The gene encodes a putative member of the Regulator of Vps4 activity in the MVB pathwa JAZ1 is a nuclear-localized protein involved ir Encodes a trypsin inhibitor involved in module PLAT/LH2 domain-containing lipoxygenase fa cold regulated gene, the 5 ' region of cor78 ha Encodes a member of the R2R3 transcription Encodes a protein that acts as an ornithine $\mathrm{N}$ Encodes a NAC-domain transcription factor. I encodes a NAC transcription factor whose ex NAC domain containing protein 47; (source: $\mathrm{Ar}$ 
AT1G69490

AT1G65690

AT4G11650

AT5G05340

AT3G26830

AT3G49780

AT5G54490

AT2G18660

AT5G59820

AT1G27730

AT1G73805

AT4G35770

AT2G29350

AT2G24850

AT2G17740

AT4G31800

AT5G22570

AT1G80840

AT5G13080

AT5G46350

AT2G35980
AT1G69490.1

AT1G65690.1

AT4G11650.1

AT5G05340.1

AT3G26830.1

AT3G49780.1

AT5G54490.1

AT2G18660.1

AT5G59820.1

AT1G27730.1

AT1G73805.1

AT4G35770.1

AT2G29350.1

AT2G24850.1

AT2G17740.1

AT4G31800.1

AT5G22570.1

AT1G80840.1

AT5G13080.1

AT5G46350.1

AT2G35980.1
Encodes a member of the NAC transcription 1

Encodes NHL6 (NDR1/HIN1-like 6). Plays an osmotin-like protein protein_coding OSMO Encodes a protein with sequence similarity to Mutations in pad3 are defective in biosynthes Phytosulfokine 3 precursor, coding for a uniqu Encodes a PINOID (PID)-binding protein cont Encodes PNP-A (Plant Natriuretic Peptide A). Encodes a zinc finger protein involved in high Related to Cys2/His2-type zinc-finger proteins Encodes SAR Deficient 1 (SARD1), a key rec Senescence-associated gene that is strongly senescence-associated gene SAG13 encodir Encodes a tyrosine aminotransferase that is $r$ VACUOLELESS GAMETOPHYTES (VLG) as Pathogen-induced transcription factor. Binds member of WRKY Transcription Factor; Grou Pathogen-induced transcription factor. Binds WRKY75 is one of several transcription factol member of WRKY Transcription Factor; Grou Encodes a protein whose sequence is similar

\section{Experiments}

\section{Name}

$\mathrm{X}$ 17days.leaf2

X17days.leaf4

ABA_1h

ABA_3h

ABA_Exp2

AgNO3

Avr_4h

Avr_8h

B.cinerea_18h

B.cinerea_48h

B.graminis

BTH_Exp1

BTH_Exp2

BTH_npr1_Exp2

coi1

Col.senescence

Cold.shoot.12h

Cold.shoot.1h

Cold.shoot.24h

Cold.shoot.3h

Cold.shoot.6h

ctr1

Drought.shoot.12h

$\begin{array}{ll}\text { Source }^{\dagger} & \text { Accession } \\ \text { NCBI GEO } & \text { GSE5630 } \\ \text { NCBI GEO } & \text { GSE5630 } \\ \text { NASCArrays } & \text { NASCARRAYS-176 } \\ \text { NASCArrays } & \text { NASCARRAYS-176 } \\ \text { NCBI GEO } & \text { GSE7112 } \\ \text { NASCArrays } & \text { NASCARRAYS-188 } \\ \text { NCBI GEO } & \text { GSE5685 } \\ \text { NCBI GEO } & \text { GSE5685 } \\ \text { ArrayExpress } & \text { E-GEOD-5684 } \\ \text { ArrayExpress } & \text { E-GEOD-5684 } \\ \text { ArrayExpress } & \text { E-GEOD-12856 } \\ \text { IMDS } & \text { BTH time course } \\ \text { IMDS } & \text { BTH experiment 2 } \\ \text { IMDS } & \text { BTH npr1 Exp2 } \\ \text { NCBI GEO } & \text { GSE65740 } \\ \text { NCBI GEO } & \text { GSE5727 } \\ \text { NCBI GEO } & \text { GSE5621 } \\ \text { NCBI GEO } & \text { GSE5621 } \\ \text { NCBI GEO } & \text { GSE5621 } \\ \text { NCBI GEO } & \text { GSE5621 } \\ \text { NCBI GEO } & \text { GSE5621 } \\ \text { ArrayExpress } & \text { E-MEXP-174 } \\ \text { NCBI GEO } & \text { GSE5624 }\end{array}$


Drought.shoot.1h

Drought.shoot.30min

Drought.shoot.3h

Drought.shoot.6h

E.cichoracearum

ein2

ET.3hr

Flg22_1h

Flg22_4h

GST_4h

$\mathrm{H} 2 \mathrm{O} 2$

HrpZ_1h

HrpZ_4h

jin1.9.MeJA

LPS_4h

MeJA

MeJA_2h

MeJA_30min

MeJA_6h

NahG

Norflurazon

NPP1 1h

NPP1_4h

OPDA

Osmotic.shoot.12h

Osmotic.shoot.1h

Osmotic.shoot.24h

Osmotic.shoot.3h

Osmotic.shoot.6h

Ozone

$P Q$ 12h

$P Q$ 24h

$P Q \_$Exp2

root_hypoxia_pCO2

root_hypoxia_pSCR

root_hypoxia_pSHR

root_hypoxia_pSUC2

root_hypoxia_pWOL

roottip_hypoxia_p35S

Rotenone_3h

Salt.root.12h

Salt.root.1h

Salt.root.24h

Salt.root.3h

Salt.root.6h

Salt.shoot.12h

Salt.shoot.24h
NCBI GEO GSE5624

NCBI GEO GSE5624

NCBI GEO GSE5624

NCBI GEO GSE5624

NCBI GEO GDS417

NCBI GEO GSE5727

NASCArrays NASCARRAYS-32

NCBI GEO GSE5615

NCBI GEO GSE5615

NCBI GEO GSE5615

NCBI GEO GSE5530

NCBI GEO GSE5615

NCBI GEO GSE5615

ArrayExpress E-MEXP-883

NCBI GEO GSE5615

ArrayExpress E-ATMX-13

ArrayExpress E-ATMX-13

ArrayExpress E-ATMX-13

ArrayExpress E-ATMX-13

NCBI GEO GSE5727

NCBI GEO GSE12887

NCBI GEO GSE5615

NCBI GEO GSE5615

NCBI GEO GSE10732

NCBI GEO GSE5622

NCBI GEO GSE5622

NCBI GEO GSE5622

NCBI GEO GSE5622

NCBI GEO GSE5622

NCBI GEO GSE5722

NASCArrays NASCARRAYS-143

NASCArrays NASCARRAYS-143

ArrayExpress E-ATMX-28

NCBI GEO GSE14578

NCBI GEO GSE14578

NCBI GEO GSE14578

NCBI GEO GSE14578

NCBI GEO GSE14578

NCBI GEO GSE14578

ArrayExpress E-MEXP-1797

NCBI GEO GSE5623

NCBI GEO GSE5623

NCBI GEO GSE5623

NCBI GEO GSE5623

NCBI GEO GSE5623

NCBI GEO GSE5623

NCBI GEO GSE5623 


\begin{tabular}{|c|c|c|}
\hline Salt.shoot.3h & NCBI GEO & GSE5623 \\
\hline Salt.shoot.6h & NCBI GEO & GSE5623 \\
\hline shoot_hypoxia_p35S & NCBI GEO & GSE14578 \\
\hline shoot_hypoxia_pRBCS & NCBI GEO & GSE14578 \\
\hline shoot_hypoxia_total & NCBI GEO & GSE14578 \\
\hline Syringolin_12h & ArrayExpress & E-MEXP-739 \\
\hline Vir_16h & NCBI GEO & GSE5685 \\
\hline Vir_24h & NCBI GEO & GSE5685 \\
\hline Vir_8h & NCBI GEO & GSE5685 \\
\hline ZAT12 & NASCArrays & NASCARRAYS-353 \\
\hline high_light_OE63 & NCBI GEO & GSE46107 \\
\hline high_light_W40 & NCBI GEO & GSE46107 \\
\hline HL_OE40 & NCBI GEO & GSE46107 \\
\hline HL_OE63 & NCBI GEO & GSE46107 \\
\hline HL_W40 & NCBI GEO & GSE46107 \\
\hline sen.JA_SA_ET.Co & NCBI GEO & GSE5727 \\
\hline sen.JA_SA_ET.Ei & NCBI GEO & GSE5727 \\
\hline sen.JA_SA_ET.NG & NCBI GEO & GSE5727 \\
\hline MLO_5 & NCBI GEO & GSE17875 \\
\hline MLO_6 & NCBI GEO & GSE17875 \\
\hline MLO_7 & NCBI GEO & GSE17875 \\
\hline X1uM_IAA_for_1h & NCBI GEO & GDS743 \\
\hline X1uM_IAA_for_3h & NCBI GEO & GDS743 \\
\hline Brassinolid_1uM_1h & NCBI GEO & GDS743 \\
\hline Brassinolid_1uM_30min & NCBI GEO & GDS743 \\
\hline Brassinolid_1uM_3h & NCBI GEO & GDS743 \\
\hline rcd1_vs_Col_control_0h & ArrayExpress & E-MTAB-662 \\
\hline rcd1_vs_Col_control_1h & ArrayExpress & E-MTAB-662 \\
\hline rcd1_vs_Col_control_2h & ArrayExpress & E-MTAB-662 \\
\hline rcd1_vs_Col_control_4h & ArrayExpress & E-MTAB-662 \\
\hline rcd1_vs_Col_control_8h & ArrayExpress & E-MTAB-662 \\
\hline rcd1_vs_Col_control_24h & ArrayExpress & E-MTAB-662 \\
\hline
\end{tabular}


(bulk/index.jsp) and represent TAIR10 Gene Annotation Data.

on Omnibus (NCBI GEO); The EMBL European Bioinformatics Institute (Array E) ird Medical School Integrated Microarray Database System (IMDS).

\section{ipe Primary Gene Symbol All Gene Symbols}

protein_coding

16);(source:Araport11) protein_coding

ted) protein. Belongs to the plant thionin (PR-13) family with the following memb d storage $2 S$ albumin superfamily protein;(source:Araport11) protein_coding 1_coding

$\mathrm{g}$

Jurce:Araport11) protein_coding

protein_coding

rotein;(source:Araport11) protein_coding

ource:Araport11) protein_coding

protein_coding

source:Araport11) protein_coding

ce:Araport11) protein_coding

ort11) protein_coding

B;(source:Araport11) protein_coding

protein_coding

rt11) protein_coding

ort11) protein_coding

רosphatidyltransferase;(source:Araport11) protein_coding

d storage 2S albumin superfamily protein;(source:Araport11) protein_coding

that interacts with the 30C02 cyst nematode effector. May play a role in host defe in;(source:Araport11) protein_coding

n_coding

ort11) protein_coding

aport11) protein_coding

ein;(source:Araport11) protein_coding

ing

irt11) protein_coding

:Araport11) protein_coding (ATBBE7) (ATBBE7)

:Araport11) protein_coding (ATBBE8) (ATBBE8)

otein involved in plant immunity.Mutants show an increase in root length under $\uparrow$ ransfer proteins. Contains a PRR domain that appears to be required for localiza rotein;(source:Araport11) protein_coding (PDH2) (PDH2)

protein_coding (SBT3.3) (SBT3.3); (ATSBT3.3)

p III protein_coding (WRKY53) (ATWRKY53); (WRKY53)

y "CHITINASE, PUTATIVE" (CHI) "CHITINASE, PUTATIVE" (CHI)

ctivity; involved in ABA catabolism. Mutant analyses show that disruption in the $g$ 
Jane-1-carboxylate (ACC) synthase (S-adenosyl-L-methionine methylthioadenos ase (PGL) thought to be involved in the oxidative pentose-phosphate pathway ( $C$ protein_coding ALTERNATIVE OXIDASE 1D (AOX1D) ALTERNATIVE OXID/ nce gene avrRpt2 and RPS2 after infection with Pseudomonas syringae pv macl e/jasmonic acid mediated signalling pathway during systemic acquired resistance ר TCF, it promotes lignin biosynthesis in response to cold stress. The mRNA is $\mathrm{C}_{1}$ is to BON1 in yeast two hybrid analyses. Its ability to bind to phospholipids is ent :omponent of the TAC1-mediated telomerase activation pathway. Acts redundan ein_coding CALCIUM EXCHANGER 7 (CAX7) (CCX1);CALCIUM EXCHAN n_coding CALMODULIN LIKE 37 (CML37) CALMODULIN LIKE 37 (CML37); n_coding CALMODULIN-LIKE 38 (CML38) n_coding CALMODULIN-LIKE 41 (CML41) CALMODULIN-LIKE 41 (CML41) ) (also named as ATL31), a RING type ubiquitin ligase that functions in the Carbr Jurce:Araport11) protein_coding CASP-LIKE PROTEIN 1D1 (CASPL1D1) ZR4-associated factor 1: AT3G44260 (CAF1a), AT5G22250 (CAF1b). Has mRN nolog (CWLP) protein_coding CELL WALL-PLASMA MEMBRANE LINKER PRC ope of chloroplasts. Provide freezing tolerance. Expression is induced by short-t ted to the chloroplast. Cor15am protects stromal proteins from aggregation und e involved in amino acid metabolism, providing the plant with cysteine and the ge kinase. protein_coding CYSTEINE-RICH RLK (RECEPTOR-LIKE PROTEIN K y CYTOCHROME P450, FAMILY 71, SUBFAMILY A, POLYPEPTIDE 13 (CYP7 ite carriers (DIC): DIC1 (AT2G22500), DIC2 (AT4G24570), DIC3 (AT5G09470). xygenase superfamily protein;(source:Araport11) protein_coding DMR6-LIKE alization-responsive and cold-induced. It is involved in priming the SAR and ISR ngle copy in Arabidopsis. It's expression is increased during light induced oxidativ sonse factor) subfamily B-3 of ERF/AP2 transcription factor family. The protein cc ning convention from the Expansin Working Group (Kende et al, 2004. Plant Mo B-LRR-conditioned resistance to avirulent pathogens and for basal resistance to involved in early defense signaling. Expression of this gene is strongly induced $c$ the formation of galactinol from UDP-galactose and myo-inositol. GolS2 transcrik protein_coding GALACTINOL SYNTHASE 3 (GolS3) GALACTINOL SYNTHA . Expression is upregulated in the shoot of cax $1 / \mathrm{cax} 3$ mutant. The mRNA is cell, the tau class of GSTs. Naming convention according to Wagner et al. (2002). , the tau class of GSTs. Naming convention according to Wagner et al. (2002). tPS3. A member of the phosphate starvation-induced glycerol-3-phosphate perm ? whose gene expression is induced by dehydration and ABA. The mRNA is cellLRR-RLK protein family. Expressin and mutant analysis revealed that it contribu y protein;(source:Araport11) protein_coding IST1-LIKE 6 (ISTL6) IST1-LI I jasmonate signaling. JAZ1 transcript levels rise in response to a jasmonate stim ating programmed cell death in plant-pathogen interactions. protein_coding amily protein;(source:Araport11) protein_coding LIPOXYGENASE 4 (LOX4) is cis-acting regulatory elements that can impart cold-regulated gene expression factor gene family. Expressed in response to potassium deprivation and auxin. I -delta-acetyltransferase, leading to the formation of $\mathrm{N}$-delta-actetylornithine. This Jositively regulates aging-induced cell death and senescence in leaves. This ge pression is induced by drought, high salt, and abscisic acid. This gene binds to $E$ aport11) protein_coding NAC DOMAIN CONTAINING PROTEIN 47 (NAC04i 
factor gene family. It is expressed in floral primordia and upregulated by AP3 an important role in the abiotic stresses-induced ABA signaling and biosynthesis, $p$ TIN 34 (OSM34) OSMOTIN 34 (OSM34);OSMOTIN 34 (ATOSM34) peroxidases that is involved in lignin biosynthesis. Loss of function mutations sh is of the indole derived phytoalexin camalexin. Encodes a cytochrome P450 enz: Ie plant peptide growth factor. Plants overexpressing this gene (under a 35S pro taining putative EF-hand calcium-binding motifs. The interaction is dependent or . PNPs are a class of systemically mobile molecules distantly related to expansin light and cold acclimation. Overexpression of this putative transcription factor in $s$ found in higher plants. Compensated for a subset of calcineurin deficiency in y $\epsilon$ julator for ICS1 (Isochorismate Synthase 1) induction and salicylic acid (SA) synt induced by phosphate starvation. Transcripts are differentially regulated at the I ig a short-chain alcohol dehydrogenase protein_coding SENESCENCE-ASSO esponsive to treatment with jasmonic acid. protein_coding TYROSINE AMINOT ; a DC1 domain containing protein that is found in the endomembrane system. I W-box sequences in vitro. Forms protein complexes with itself and with WRKY4 p III protein_coding WRKY DNA-BINDING PROTEIN 38 (WRKY38) ARABIDI $\mathrm{W}$-box sequences in vitro. Forms protein complexes with itself and with WRKY4 rs induced during $\mathrm{Pi}$ deprivation. It is nuclear localized and regulated differentiall) p II-c protein_coding WRKY DNA-BINDING PROTEIN 8 (WRKY8) WRKY DI to tobacco hairpin-induced gene (HIN1) and Arabidopsis non-race specific disee

\section{Link}

https://www.ncbi.nlm.nih.gov/geo/query/acc.cgi?acc=GSE5630 https://www.ncbi.nlm.nih.gov/geo/query/acc.cgi?acc=GSE5630 http://www.webcitation.org/getfile?fileid=c2cde11f2c71cfb474c58964754c6aecc: http://www.webcitation.org/getfile?fileid=c2cde11f2c71 cfb474c58964754c6aecc: https://www.ncbi.nlm.nih.gov/geo/query/acc.cgi?acc=GSE7112 http://www.webcitation.org/getfile?fileid=c2cde11f2c71cfb474c58964754c6aecc: https://www.ncbi.nlm.nih.gov/geo/query/acc.cgi?acc=GSE5685 https://www.ncbi.nlm.nih.gov/geo/query/acc.cgi?acc=GSE5685 https://www.ebi.ac.uk/arrayexpress/experiments/E-GEOD-5684/ https://www.ebi.ac.uk/arrayexpress/experiments/E-GEOD-5684/ https://www.ebi.ac.uk/arrayexpress/experiments/E-GEOD-12856/ https://pga.mgh.harvard.edu/Parabiosys/resources/microarrays/integrated_micrc https://pga.mgh.harvard.edu/Parabiosys/resources/microarrays/integrated_micrc https://pga.mgh.harvard.edu/Parabiosys/resources/microarrays/integrated_micrc https://www.ncbi.nlm.nih.gov/geo/query/acc.cgi?acc=GSE65740 https://www.ncbi.nlm.nih.gov/geo/query/acc.cgi?acc=GSE5727 https://www.ncbi.nlm.nih.gov/geo/query/acc.cgi?acc=GSE5621 https://www.ncbi.nlm.nih.gov/geo/query/acc.cgi?acc=GSE5621 https://www.ncbi.nlm.nih.gov/geo/query/acc.cgi?acc=GSE5621 https://www.ncbi.nlm.nih.gov/geo/query/acc.cgi?acc=GSE5621 https://www.ncbi.nlm.nih.gov/geo/query/acc.cgi?acc=GSE5621 https://www.ebi.ac.uk/arrayexpress/experiments/E-MEXP-174/ https://www.ncbi.nlm.nih.gov/geo/query/acc.cgi?acc=GSE5624 
https://www.ncbi.nlm.nih.gov/geo/query/acc.cgi?acc=GSE5624 https://www.ncbi.nlm.nih.gov/geo/query/acc.cgi?acc=GSE5624 https://www.ncbi.nlm.nih.gov/geo/query/acc.cgi?acc=GSE5624 https://www.ncbi.nlm.nih.gov/geo/query/acc.cgi?acc=GSE5624 https://www.ncbi.nlm.nih.gov/sites/GDSbrowser?acc=GDS417 http://www.webcitation.org/getfile?fileid=c2cde11f2c71cfb474c58964754c6aecc: http://www.webcitation.org/getfile?fileid=c2cde11f2c71cfb474c58964754c6aecc: https://www.ncbi.nlm.nih.gov/geo/query/acc.cgi?acc=GSE5615 https://www.ncbi.nlm.nih.gov/geo/query/acc.cgi?acc=GSE5615 https://www.ncbi.nlm.nih.gov/geo/query/acc.cgi?acc=GSE5615 https://www.ncbi.nlm.nih.gov/geo/query/acc.cgi?acc=GSE5530 https://www.ncbi.nlm.nih.gov/geo/query/acc.cgi?acc=GSE5615 https://www.ncbi.nlm.nih.gov/geo/query/acc.cgi?acc=GSE5615 https://www.ebi.ac.uk/arrayexpress/experiments/E-MEXP-883/ https://www.ncbi.nlm.nih.gov/geo/query/acc.cgi?acc=GSE5615 https://www.ebi.ac.uk/arrayexpress/experiments/E-ATMX-13/ https://www.ebi.ac.uk/arrayexpress/experiments/E-ATMX-13/ https://www.ebi.ac.uk/arrayexpress/experiments/E-ATMX-13/ https://www.ebi.ac.uk/arrayexpress/experiments/E-ATMX-13/ https://www.ncbi.nlm.nih.gov/geo/query/acc.cgi?acc=GSE65740 https://www.ncbi.nlm.nih.gov/geo/query/acc.cgi?acc=GSE3709 https://www.ncbi.nlm.nih.gov/geo/query/acc.cgi?acc=GSE5615 https://www.ncbi.nlm.nih.gov/geo/query/acc.cgi?acc=GSE5615 https://www.ncbi.nlm.nih.gov/geo/query/acc.cgi?acc=GSE10732 https://www.ncbi.nlm.nih.gov/geo/query/acc.cgi?acc=GSE5622 https://www.ncbi.nlm.nih.gov/geo/query/acc.cgi?acc=GSE5622 https://www.ncbi.nlm.nih.gov/geo/query/acc.cgi?acc=GSE5622 https://www.ncbi.nlm.nih.gov/geo/query/acc.cgi?acc=GSE5622 https://www.ncbi.nlm.nih.gov/geo/query/acc.cgi?acc=GSE5622 https://www.ncbi.nlm.nih.gov/geo/query/acc.cgi?acc=GSE5722 http://www.webcitation.org/getfile?fileid=c2cde11f2c71cfb474c58964754c6aecc: http://www.webcitation.org/getfile?fileid=c2cde11f2c71cfb474c58964754c6aecc: https://www.ebi.ac.uk/arrayexpress/experiments/E-ATMX-28/ https://www.ncbi.nlm.nih.gov/geo/query/acc.cgi?acc=GSE14578 https://www.ncbi.nlm.nih.gov/geo/query/acc.cgi?acc=GSE14578 https://www.ncbi.nlm.nih.gov/geo/query/acc.cgi?acc=GSE14578 https://www.ncbi.nlm.nih.gov/geo/query/acc.cgi?acc=GSE14578 https://www.ncbi.nlm.nih.gov/geo/query/acc.cgi?acc=GSE14578 https://www.ncbi.nlm.nih.gov/geo/query/acc.cgi?acc=GSE14578 https://www.ebi.ac.uk/arrayexpress/experiments/E-MEXP-1797/ https://www.ncbi.nlm.nih.gov/geo/query/acc.cgi?acc=GSE5623 https://www.ncbi.nlm.nih.gov/geo/query/acc.cgi?acc=GSE5623 https://www.ncbi.nlm.nih.gov/geo/query/acc.cgi?acc=GSE5623 https://www.ncbi.nlm.nih.gov/geo/query/acc.cgi?acc=GSE5623 https://www.ncbi.nlm.nih.gov/geo/query/acc.cgi?acc=GSE5623 https://www.ncbi.nlm.nih.gov/geo/query/acc.cgi?acc=GSE5623 https://www.ncbi.nlm.nih.gov/geo/query/acc.cgi?acc=GSE5623 
https://www.ncbi.nlm.nih.gov/geo/query/acc.cgi?acc=GSE5623 https://www.ncbi.nlm.nih.gov/geo/query/acc.cgi?acc=GSE5623 https://www.ncbi.nlm.nih.gov/geo/query/acc.cgi?acc=GSE14578 https://www.ncbi.nlm.nih.gov/geo/query/acc.cgi?acc=GSE14578 https://www.ncbi.nlm.nih.gov/geo/query/acc.cgi?acc=GSE14578 https://www.ebi.ac.uk/arrayexpress/experiments/E-MEXP-739/ https://www.ncbi.nlm.nih.gov/geo/query/acc.cgi?acc=GSE5685 https://www.ncbi.nlm.nih.gov/geo/query/acc.cgi?acc=GSE5685 https://www.ncbi.nlm.nih.gov/geo/query/acc.cgi?acc=GSE5685 http://www.webcitation.org/getfile?fileid=c2cde11f2c71cfb474c58964754c6aecc: https://www.ncbi.nlm.nih.gov/geo/query/acc.cgi?acc=GSE46107 https://www.ncbi.nlm.nih.gov/geo/query/acc.cgi?acc=GSE46107 https://www.ncbi.nlm.nih.gov/geo/query/acc.cgi?acc=GSE46107 https://www.ncbi.nlm.nih.gov/geo/query/acc.cgi?acc=GSE46107 https://www.ncbi.nlm.nih.gov/geo/query/acc.cgi?acc=GSE46107 https://www.ncbi.nlm.nih.gov/geo/query/acc.cgi?acc=GSE5727 https://www.ncbi.nlm.nih.gov/geo/query/acc.cgi?acc=GSE5727 https://www.ncbi.nlm.nih.gov/geo/query/acc.cgi?acc=GSE5727 https://www.ncbi.nlm.nih.gov/geo/query/acc.cgi?acc=GSE17875 https://www.ncbi.nlm.nih.gov/geo/query/acc.cgi?acc=GSE17875 https://www.ncbi.nlm.nih.gov/geo/query/acc.cgi?acc=GSE17875 https://www.ncbi.nlm.nih.gov/sites/GDSbrowser?acc=GDS743 https://www.ncbi.nlm.nih.gov/sites/GDSbrowser?acc=GDS743 https://www.ncbi.nlm.nih.gov/sites/GDSbrowser?acc=GDS743 https://www.ncbi.nlm.nih.gov/sites/GDSbrowser?acc=GDS743 https://www.ncbi.nlm.nih.gov/sites/GDSbrowser?acc=GDS743 https://www.ebi.ac.uk/arrayexpress/experiments/E-MTAB-662/ https://www.ebi.ac.uk/arrayexpress/experiments/E-MTAB-662/ https://www.ebi.ac.uk/arrayexpress/experiments/E-MTAB-662/ https://www.ebi.ac.uk/arrayexpress/experiments/E-MTAB-662/ https://www.ebi.ac.uk/arrayexpress/experiments/E-MTAB-662/ https://www.ebi.ac.uk/arrayexpress/experiments/E-MTAB-662/ 
(press);

ers: At1g66100, At5g36910, At1g72260, At2g15010, At1g12663, At1g12660. prote

ənse. protein_coding

$\mathrm{NO}$ stress and reduction in root length under $\mathrm{H} 2 \mathrm{O} 2$ stress conditions. Mutants show in tion to the chloroplast. protein_coding (AZI3) (AZI3)

ene results in more drought tolerance whereas overexpression results in increased tre 
ine-lyase, EC 4.4.1.14) gene family The mRNA is cell-to-cell mobile. protein_codi JPPP). protein_coding 6-PHOSPHOGLUCONOLACTONASE 5 (PGL5) 6-PHOSI ISE 1D (AOX1D)

ılicola strain ES4326 carrying avrRpt2 protein_coding AVRRPT2-INDUCED GENE 1 ə based on expression analyses. protein_coding BASIC CHITINASE (HCHIB) (CF ell-to-cell mobile. protein_coding BLUE-COPPER-BINDING PROTEIN (BCB) BLI lanced by calcium ions. Involved in maintaining cell homeostasis. protein_coding BOI tly with BT3 and BT1 during female gametophyte development and with BT3 during $\mathrm{m}$ GER 7 (CAX7)

(ATCML37);CALMODULIN LIKE 39 (CML39)

Jn/Nitrogen response for growth phase transition in Arabidopsis seedlings. The mRN $f$ CASP-LIKE PROTEIN 1D1 (CASPL1D1)

A deadenylation activity. Also plays a role in plant defense responses. protein_cc JTEIN (CWLP) CELL WALL-PLASMA MEMBRANE LINKER PROTEIN (CWLP) erm cold-treatment, water deprivation, and abscisic acid treatment. protein_coding er various stress conditions. Constitutive expression increases freezing tolerance in $\mathrm{pr}$ zneration of precursors of ethylene biosynthesis. mRNA levels are elevated in respon INASE) 7 (CRK7) CYSTEINE-RICH RLK (RECEPTOR-LIKE PROTEIN KINASE) : '1A13) CYTOCHROME P450, FAMILY 71, SUBFAMILY A, POLYPEPTIDE 13 (CY The mRNA is cell-to-cell mobile. protein_coding DICARBOXYLATE CARRIER 2 (DII : OXYGENASE 1 (DLO1) DMR6-LIKE OXYGENASE 1 (DLO1)

responses, specifically in propagating the cell-to-cell mobile signal. protein_coding $E$ 'e stress ,drought stress and also during senescence. Promoter contains two AGL15 k sntains one AP2 domain. There are 18 members in this subfamily including ATERF-1, I Bio). Involved in the formation of nematode-induced syncytia in roots of Arabidopsis invasive virulent pathogens. Functions in an EDS1-regulated but SA-independent me Juring leaf senescence. It is a target of the transcription factor AtWRKY6. protein_c tt levels rise in response to methyl viologen, an oxidative damage-inducing agent. Pla ISE 3 (GolS3);GALACTINOL SYNTHASE 3 (AtGoIS3)

to-cell mobile. protein_coding GLUCOSE-6-PHOSPHATE/PHOSPHATE TRANS protein_coding GLUTATHIONE S-TRANSFERASE TAU 11 (GSTU11) GLUTA protein_coding GLUTATHIONE S-TRANSFERASE TAU 4 (GSTU4) GLUTATHIOI ıease gene family: AT3G47420(G3Pp1), AT4G25220(G3Pp2), AT1G30560(G3Pp3), , to-cell mobile. protein_coding HISTONE H1-3 (HIS1-3) HISTONE H1-3 (HIS1-3', tes to the interaction between Arabidopsis and Hyaloperonospora arabidopsidis. and KE 6 (ISTL6)

lulus. JAZ1 can interact with the COI1 F-box subunit of an SCF E3 ubiquitin ligase in : KUNITZ TRYPSIN INHIBITOR 1 (KTI1) KUNITZ TRYPSIN INHIBITOR 1 (KTI1);A ARABIDOPSIS THALIANA LIPOXYGENASE 4 (ATLOX4);LIPOXYGENASE 4 (LOX، The mRNA is cell-to-cell mobile. protein_coding LOW-TEMPERATURE-INDUCED 7 nvolved in lateral root development. Interacts with ARF7 and regulates the expression ; compound is likely used in plant defense and levels of it are increased in Arabidopsis ne is upregulated in response to salt stress in wildtype as well as NTHK1 transgenic li :RD1 promoter in vitro. protein_coding NAC DOMAIN CONTAINING PROTEIN 19 7) NAC DOMAIN CONTAINING PROTEIN 47 (anac047);SPEEDY HYPONASTIC 
d PI. Its expression is associated with leaf senescence. The mRNA is cell-to-cell mob articularly during seed germination and early seedling development. protein_coding

ow abnormal development of xylem fibers and reduced levels of lignin biosynthetic en yme that catalyzes the conversion of dihydrocamalexic acid to camalexin. The mRNA moter), develop normal cotyledons and hypocotyls but their growth, in particular that $c$ it the presence of calcium. mRNA expression is up-regulated by auxin. Not a phosphol Is; their biological role has remained elusive. PNP-A contains a signal peptide domain zreases the expression level of 9 cold-responsive genes and represses the expressior zast. Salt tolerance produced by ZAT10 appeared to be partially dependent on ENA1/ hesis. protein_coding SAR DEFICIENT 1 (SARD1) SAR DEFICIENT 1 (SARD1) evel of mRNA stability at different times of day. mRNAs are targets of the mRNA degr. CIATED GENE 13 (SAG13) SENESCENCE-ASSOCIATED GENE 13 (SAG13) RANSFERASE 3 (TAT3) TYROSINE AMINOTRANSFERASE 3 (TAT3);TYROSIN $t$ is essential for both female and male gametophyte development. protein_coding $V t$ 0 and WRKY60. Constitutive expression of WRKY18 enhanced resistance to P. syrir OPSIS THALIANA WRKY DNA-BINDING PROTEIN 38 (ATWRKY38);WRKY DNA-BI 0 and WRKY60. Coexpression with WRKY18 or WRKY60 made plants more suscept I during Pi starvation. RNAi mediated suppression of WRKY75 made the plants more NA-BINDING PROTEIN 8 (WRKY8);ARABIDOPSIS THALIANA WRKY DNA-BINDINC ise resistance gene (NDR1). Expression of this gene is induced by cucumber mosaic

7586986

7586986

7586986

Jarray_database_system.php

Jarray_database_system.php

Jarray_database_system.php 
7586986

7586986

7586986

7586986 
7586986 
in_coding

creases in defense responses to pathogens including hypersensitive lesions, increased

ænspiration rate and reduced drought tolerance. Gene involved in postgermination growtl 
ng 1-AMINOCYCLOPROPANE-1-CARBOXYLIC ACID (ACC) SYNTHASE 6 (ACS6) । PHOGLUCONOLACTONASE 5 (PGL5)

(AIG1) AVRRPT2-INDUCED GENE 1 (AIG1)

II-B);BASIC CHITINASE (ATHCHIB);PATHOGENESIS-RELATED 3 (PR3);PATHOGEN UE-COPPER-BINDING PROTEIN (ATBCB);BLUE-COPPER-BINDING PROTEIN (BCB) $N$ ASSOCIATION PROTEIN 1 (BAP1) BON ASSOCIATION PROTEIN 1 (BAP1) ale gametophyte development. BT2 also mediates multiple responses to nutrients, stres

I is cell-to-cell mobile. protein_coding CARBON/NITROGEN INSENSITIVE 1 (CNI1) sding CCR4- ASSOCIATED FACTOR 1A (CAF1a) CCR4- ASSOCIATED FACTOR 1 COLD REGULATED 314 INNER MEMBRANE 1 (COR413IM1) COLD REGULATED 3 otoplasts in vitro and chloroplasts in vivo. NMR and $x$-ray diffraction studies suggest tha se to wounding. protein_coding CORONATINE INDUCED 1 (CORI3) JASMONIC 7 (CRK7)

'P71A13)

C2) DICARBOXYLATE CARRIER 2 (DIC2)

:ARLY ARABIDOPSIS ALUMINUM INDUCED 1 (EARLI1) EARLY ARABIDOPSIS ALU jinding sites. protein_coding ENESCENCE-ASSOCIATED AND QQS-RELATED ( $S$ ATERF-2, AND ATERF-5. protein_coding ETHYLENE RESPONSE FACTOR 104 । thaliana. protein_coding EXPANSIN A8 (EXPA8) (EXP8); (ATEXP8);EXPANSIN A chanism that promotes resistance and cell death at pathogen infection sites. protein oding FLG22-INDUCED RECEPTOR-LIKE KINASE 1 (FRK1) SE- NESCENCE-INDI nts over-expressing GolS2 have increased tolerance to salt, chilling, and high-light stres:

LOCATOR 2 (GPT2) GLUCOSE-6-PHOSPHATE/PHOSPHATE TRANSLOCATOR 2 ITHIONE S-TRANSFERASE TAU 11 (GSTU11);GLUTATHIONE S-TRANSFERASE TA VE S-TRANSFERASE TAU 4 (ATGSTU4);GLUTATHIONE S-TRANSFERASE TAU 4 (C. 4T4G17550(G3Pp4) and AT2G13100(G3Pp5). Its expression is responsive to phosphat 1

The mRNA is cell-to-cell mobile. protein_coding IMPAIRED OOMYCETE SUSCEPTIB

a yeast-two-hybrid assay only in the presence of jasmonate-isoleucine (JA-ILE) or coron RABIDOPSIS THALIANA KUNITZ TRYPSIN INHIBITOR 1 (ATKTI1)

4)

'8 (LTI78) COLD REGULATED 78 (COR78);LOW-TEMPERATURE-INDUCED 78 (L' I of some auxin responsive genes. protein_coding MYB DOMAIN PROTEIN 77 (MYB77 ; plants in response to MeJA and ABA. The mRNA is cell-to-cell mobile. protein_coding nes although in the latter case the induction was drastically reduced. It was also upregul (NAC019) (ANAC19);NAC DOMAIN CONTAINING PROTEIN 19 (NAC019);NAC DI GROWTH (SHYG);SPEEDY HYPONASTIC GROWTH (SHG);NAC DOMAIN CONTAIN 

NDR1/HIN1-LIKE 6 (NHL6) NDR1/HIN1-LIKE 6 (NHL6)

xymes. protein_coding PEROXIDASE 52 (PRX52) PEROXIDASE 52 (PRX52) is cell-to-cell mobile. protein_coding PHYTOALEXIN DEFICIENT 3 (PAD3) (CYP71 If their roots, was faster than that of wildtype. protein_coding PHYTOSULFOKINE $4 \mathrm{P}$ rylation target of PID, likely acts upstream of PID to regulate the activity of this protein in and is secreted into the extracellular space. Co-expression analyses using microarray c 7 level of 15 cold-responsive genes, including CBF genes. Also, lines overexpressing thi: PMR2, a P-type ATPase required for $\mathrm{Li}+$ and $\mathrm{Na}+$ efflux in yeast. The protein is localizec

adation pathway mediated by the downstream (DST) instability determinant. protein_c

\section{E AMINOTRANSFERASE (TAT)}

ACUOLELESS GAMETOPHYTES (VLG) VACUOLELESS GAMETOPHYTES (VLG) Igae, but its coexpression with WRKY40 or WRKY60 made plants more susceptible to $b$ NDING PROTEIN 38 (WRKY38)

ible to both $P$. syringae and B. cinerea. WRKY18, WRKY40, and WRKY60 have partially susceptible to Pi stress as indicated by the higher accumulation of anthocyanin during à PROTEIN 8 (ATWRKY8)

virus, spermine and during senescence. The gene product is localized to the chloroplast 


resistance and induction of SAR genes. protein_coding (ATHMAD1) (ATHMAD1

ר. Plant P450 CYP707A3, ABA 8'-hydroxylase, binds enantioselectively (+)-ABA but not 
(ATACS6);1-AMINOCYCLOPROPANE-1-CARBOXYLIC ACID (ACC) SYNTHASE 6 (AC

ESIS-RELATED 3 (PR-3); (B-CHI);BASIC CHITINASE (HCHIB)

।;SENESCENCE ASSOCIATED GENE 14 (SAG14);BLUE COPPER BINDING PROTEII

ses, and hormones. protein_coding BTB AND TAZ DOMAIN PROTEIN 2 (bt2) BTB

CARBON/NITROGEN INSENSITIVE 1 (CNI1);ARABIDOPSIS TOXICOS EN LEVADUF IA (CAF1a);CCR4- ASSOCIATED FACTOR 1A (AtCAF1a)

14 INNER MEMBRANE 1 (COR413IM1);COLD REGULATED 414 THYLAKOID MEMBF $t$ COR15a alters the intrinsic curvature of the inner membrane of chloroplast envelope. I ; ACID RESPONSIVE 2 (JR2);CORONATINE INDUCED 1 (CORI3)

MINUM INDUCED 1 (EARLI1); (pEARLI 1)

iAQR) ENESCENCE-ASSOCIATED AND QQS-RELATED (SAQR)

(ERF104) ETHYLENE RESPONSE FACTOR 104 (ERF104)

8 (EXPA8); (ATHEXP ALPHA 1.11);EXPANSIN A8 (ATEXPA8)

coding FLAVIN-DEPENDENT MONOOXYGENASE 1 (FMO1) FLAVIN-DEPENDENT $\Lambda$ JCED RECEPTOR-LIKE KINASE (SIRK);FLG22-INDUCED RECEPTOR-LIKE KINASE s. protein_coding GALACTINOL SYNTHASE 2 (GoIS2) GALACTINOL SYNTHASE 2 (

(GPT2);ARABIDOPSIS GLUCOSE-6-PHOSPHATE/PHOSPHATE TRANSLOCATOR 2 U 11 (ATGSTU11)

iSTU4);GLUTATHIONE S-TRANSFERASE 22 (GST22)

$\mathrm{e}(\mathrm{Pi})$ and not phosphite (Phi) in roots and shoots. protein_coding GLYCEROL-3-PH

ILITY 1 (IOS1) IMPAIRED OOMYCETE SUSCEPTIBILITY 1 (IOS1)

atine. Application of jasmonate methyl ester to Arabidopsis roots reduces the levels of a

II78); (LTI140);RESPONSIVE TO DESICCATION 29A (RD29A)

) MYB DOMAIN PROTEIN 77 (MYB77)

I N-ACETYLTRANSFERASE ACTIVITY 1 (NATA1) N-ACETYLTRANSFERASE ACTIV ated by $A B A, A C C$ and NAA treatment, although in the latter two cases, the induction oC OMAIN CONTAINING PROTEIN 19 (ANAC019)

IING PROTEIN 47 (NAC047) 
B15);PHYTOALEXIN DEFICIENT 3 (PAD3)

RECURSOR (PSK4) PHYTOSULFOKINE 4 PRECURSOR (ATPSK4);PHYTOSULF response to changes in calcium levels. protein_coding PINOID-BINDING PROTEIN lata suggest that PNP-A may function as a component of plant defence response and $S$ $s$ gene exhibits a small but reproducible increase in freeze tolerance. Because of the ref $x$ to the nucleus, acts as a transcriptional repressor and is responsive to chitin oligomers oding SENESCENCE 1 (SEN1) SENESCENCE 1 (SEN1);DARK INDUCIBLE 1 (DIN

oth P. syringae and B. cinerea. WRKY18, WRKY40, and WRKY60 have partially redun $\checkmark$ redundant roles in response to the hemibiotrophic bacterial pathogen Pseudomonas s) Pi starvation. protein_coding WRKY DNA-BINDING PROTEIN 75 (WRKY75) AR/ . The mRNA is cell-to-cell mobile. protein_coding YELLOW-LEAF-SPECIFIC GENE $~ \subseteq$ 


I)

(-)-ABA, whereas the enzyme binds both enantiomers of AHI1 (a structural ABA analogı 
;S6)

$\checkmark(\mathrm{BCB})$

AND TAZ DOMAIN PROTEIN 2 (bt2); (ATBT2)

IA 31 (ATL31)

IANE 1 (COR414-TM1);COLD REGULATED 314 THYLAKOID MEMBRANE 1 (COR41: Late Embryogenesis abundant protein (LEA). Protects chloroplast membranes during fre

1ONOOXYGENASE 1 (FMO1)

1 (FRK1)

AtGolS2);GALACTINOL SYNTHASE 2 (GolS2)

: (ATGPT2)

OSPHATE PERMEASE 1 (G3Pp1) PHOSPHATE STARVATION-INDUCED GENE 3 (A'

JAZ1:GUS fusion protein, presumably by stimulating ubiquitin-proteasome-mediated $d$

'ITY 1 (NATA1)

:curred relatively late when compared with $\mathrm{NaCl}$ or $\mathrm{ABA}$ treatments. Note: this protein $(\mathrm{A}$ 
VAP);NAC-LIKE, ACTIVATED BY AP3/PI (ATNAP)

OKINE 4 PRECURSOR (PSK4); (ATPSK3 (FORMER SYMBOL))

1 (PBP1) PINOID-BINDING PROTEIN 1 (PBP1)

$A R$ in particular, and could be classified as a newly identified PR protein. It is stress resk ression of the CBF genes by the overexpression of this gene, the authors speculate tha . Also involved in response to photooxidative stress. protein_coding SALT TOLERANCE 1);ARABIDOPSIS THALIANA SENESCENCE 1 (ATSEN1);SENESCENCE ASSOCIATE

dant roles in response to the hemibiotrophic bacterial pathogen Pseudomonas syringae /ringae and the necrotrophic fungal pathogen Botrytis cinerea, with WRKY18 playing a $n$ IBIDOPSIS THALIANA WRKY DNA-BINDING PROTEIN 75 (ATWRKY75);WRKY DNA-

I (YLS9) YELLOW-LEAF-SPECIFIC GENE 9 (YLS9);ARABIDOPSIS NDR1/HIN1-LIK 


ıe used as ABA 8'-hydroxylase competitive inhibitor). protein_coding "CYTOCHROME 
3-TM1)

sezing. protein_coding COLD-REGULATED 15A (COR15A) COLD-REGULATED 15/

TPS3);PHOSPHATE STARVATION-INDUCED GENE 3 (PS3);GLYCEROL-3-PHOSPH ،

egradation. The Jas domain appears to be important for JAZ1-COI1 interactions in the $p$

tNAC6) on occasion has also been referred to as AtNAC2, not to be confused with the $A$ 
Jonsive and can enhance its own expression. protein_coding PLANT NATRIURETIC $t$ this gene may be involved in negative regulatory circuit of the CBF pathway. The $m R N$, ミZINC FINGER (STZ) (ZAT10);SALT TOLERANCE ZINC FINGER (STZ)

:D GENE 1 (SEN1)

and the necrotrophic fungal pathogen Botrytis cinerea, with WRKY18 playing a more im רore important role than the other two. The mRNA is cell-to-cell mobile. protein_coding -BINDING PROTEIN 75 (WRKY75)

:E 10 (ATNHL10);NDR1/HIN1-LIKE (NHL10) 


I (COR15A); (ATCOR15A); (COR15)

ATE PERMEASE 1 (AtG3Pp1);GLYCEROL-3-PHOSPHATE PERMEASE 1 (G3Pp1)

resence of coronatine. Two positive residues (R205 and R206) in the Jas domain showr ItNAC2 found at locus AT3G15510. protein_coding NAC DOMAIN CONTAINING PRC 
PEPTIDE A (PNP-A) EXPANSIN-LIKE B3 PRECURSOR (EXLB3);PLANT NATRIUR A is cell-to-cell mobile. protein_coding RESPONSIVE TO HIGH LIGHT 41 (RHL41)

portant role than the other two. The mRNA is cell-to-cell mobile. protein_coding WRKY WRKY DNA-BINDING PROTEIN 40 (WRKY40) (ATWRKY40);WRKY DNA-BINDINC 


E P450, FAMILY 707, SUBFAMILY A, POLYPEPTIDE 3" (CYP707A3) 
I to be important for coronatine -dependent COI1 binding are not required for binding AtI ITEIN 6 (NAC6) NAC DOMAIN CONTAINING PROTEIN 2 (ATNAC2);ORESARA 1 (OF 


\section{ETIC PEPTIDE A (PNP-A); (AtPNP-A)}

(ZAT12); (ATZAT12);RESPONSIVE TO HIGH LIGHT 41 (RHL41)

' DNA-BINDING PROTEIN 18 (WRKY18) ARABIDOPSIS THALIANA WRKY DNA-BIN خ̀ PROTEIN 40 (WRKY40) 



UYC2. The mRNA is cell-to-cell mobile. protein_coding JASMONATE-ZIM-DOMAIR 2E1);NAC DOMAIN CONTAINING PROTEIN 6 (NAC6);NAC DOMAIN CONTAINING P 
JDING PROTEIN 18 (ATWRKY18);WRKY DNA-BINDING PROTEIN 18 (WRKY18) 



V PROTEIN 1 (JAZ1) JASMONATE-ZIM-DOMAIN PROTEIN 1 (JAZ1); (ATJAZ1); (TIF' ROTEIN 6 (ATNAC6);NAC DOMAIN CONTAINING PROTEIN 2 (NAC2);ARABIDOPSI 




Y10A)

S NAC DOMAIN CONTAINING PROTEIN 92 (ANAC092) 